Portland State University

PDXScholar

5-4-1994

\title{
High-frequency Analog Voltage Converter Design
}

Ping Xu

Portland State University

Follow this and additional works at: https://pdxscholar.library.pdx.edu/open_access_etds

Part of the Electrical and Computer Engineering Commons Let us know how access to this document benefits you.

\section{Recommended Citation}

Xu, Ping, "High-frequency Analog Voltage Converter Design" (1994). Dissertations and Theses. Paper 4891.

https://doi.org/10.15760/etd.6767

This Thesis is brought to you for free and open access. It has been accepted for inclusion in Dissertations and Theses by an authorized administrator of PDXScholar. Please contact us if we can make this document more accessible: pdxscholar@pdx.edu. 


\section{THESIS APPROVAL}

The abstract and dissertation of Ping Xu for the Master of Science in Electrical and Computer Engineering were presented on May 4, 1994, and accepted by the thesis committee and the department.

COMMITTEE APPROVALS:

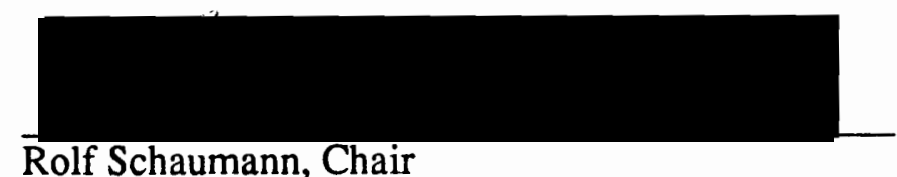

Rolf Schaumann, Chair

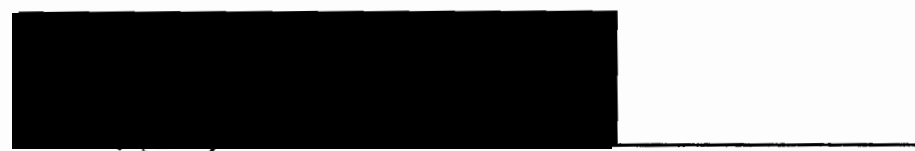

W. Robert Daasch

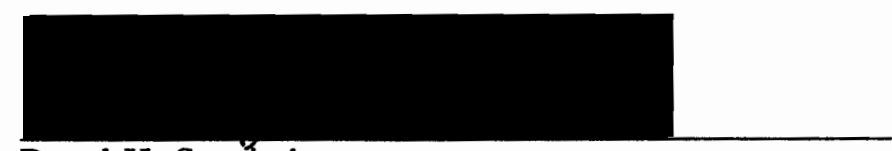

Pavel K. Smejtek

DEPARTMENT APPROVAL:

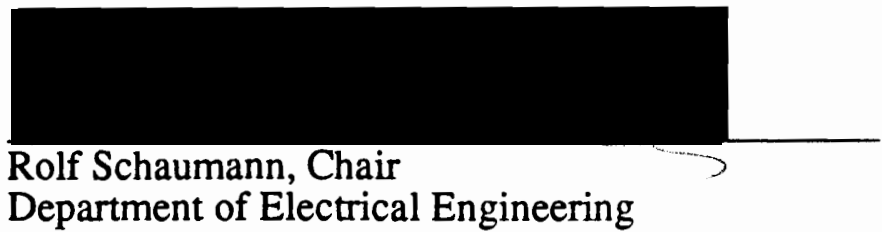

$* * * * * * * * * * * * * * * * * * * * * * * * * * * * * * * * * * * * * * * * * * * * * * * * * * * * * * * * * * * * * * * * * * * * * * * * *$

\section{ACCEPTED FOR PORTLAND STATE UNIVERSITY BY THE LIBRARY}

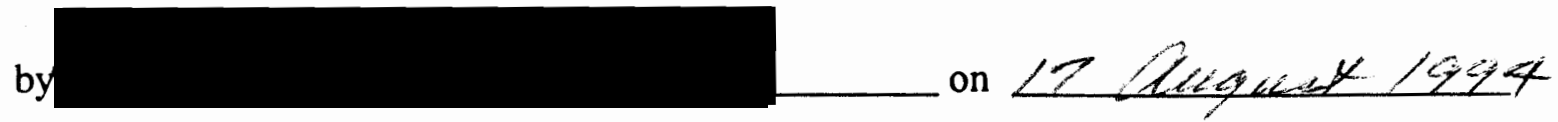




\begin{abstract}
:
An abstract of the thesis of Ping $\mathrm{Xu}$ for the Master of Science in Electrical and Computer Engineering on May 4, 1994.
\end{abstract}

Title: High-Frequency Analog Voltage Converter Design

For many high-speed, high-performance circuits, purely differential inputs are needed. This project focuses on building high-speed voltage converters which can transfer a single-ended signal to a purely differential signal, or a differential input signal to a single-ended signal.

Operational transconductance amplifier (OTAs) techniques are widely used in high-speed continuous-time integrated analog signal processing (ASP) circuits because resistors, inductors, integrators, buffers, multipliers and filters can be built by OTAs and capacitors. Taking advantage of OTAs, very-high-speed voltage converters are designed in CMOS technology. These converters can work in a frequency range from $\mathrm{DC}(\mathrm{OHz})$ up to $100 \mathrm{MHz}$ and higher, and keep low distortion over a $\pm 0.5 \mathrm{~V}$ input range. They can replace transformers so that designing fully integrated differential circuits becomes possible.

The designs are based on a MOSIS $2 \mu \mathrm{m}$ n-well process. SPICE simulations of these designs are given. The circuit was laid out with MAGIC layout tools and fabricated through MOSIS. The chip was measured at PSU and Intel circuit labs and the experimental results show the correctness of the designs. 
HIGH-FREQUENCY ANALOG VOLTAGE CONVERTER DESIGN

by

PING XU

A thesis submitted in partial fulfillment of the requirements for the degree of

\author{
MASTER OF SCIENCE \\ in \\ ELECTRICAL AND COMPUTER ENGINEERING
}

Portland State University

1994 


\section{ACKNOWLEDGEMENTS}

I am very thankful to my advisor and friend, Dr. Rolf Schaumann. He taught me how to do research and spent a lot of time to guide and help me patiently to finish many projects.

I would like to thank Dr. Robert Daasch, who gave me solid circuit design knowledge by his exciting classes and helped me with the layout of the circuit. The same appreciation is given to Dr. Van Halen.

Thanks to my friends and the faculty and staff of the Electrical Engineering Department for their friendly support and encouragement in many ways.

I want to give special thanks to my parents and my sisters. Though they do not stay with me, they always love me, are worried and joyful for me, and encourage me to work and study hard. 


\section{TABLE OF CONTENTS}

\section{PAGE}

ACKNOWLEDGEMENTS _........................................................................ iii

LIST OF TABLES …............................................................................ vi

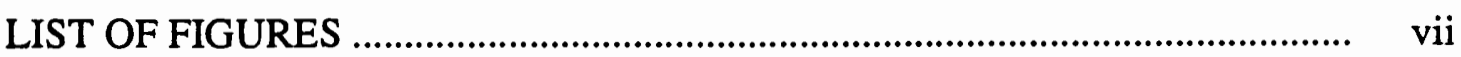

\section{CHAPTER}

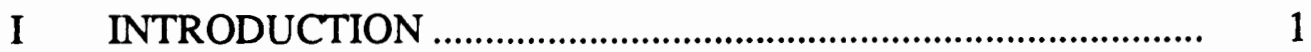

II OTA DESIGN _......................................................................... 4

$2.1 \quad$ Basic OTA Cells .................................................................. 4

2.2 Differential Input (Output) OTA Design ............................. 10

2.3 Differential to Single-Ended Output OTA Design .............. 20

2.4 Non-offset Single-Ended OTA Design ............................... 20

III BUFFER DESIGN .................................................................. 25

3.1 Introduction ................................................................ 25

3.2 Circuit Description ........................................................... 26

3.3 Simulation Results .......................................................... 29

IV The VOLTAGE CONVERTER DESIGN ..................................... 32

4.1 The First Initial Design of a Converter ................................ 32

4.2 The Second Initial Design of a Converter ............................ 34

4.3 Improved Cascade Converter Design ................................... 36

$\mathrm{V} \quad$ LAYOUT OF THE CONVERTER CHIP ..................................... 41

5.1 MOSIS Process and the Layout Tool .................................. 41

5.2 Latch-up Prevention ........................................................... 42 
5.3 Reducing the Parasitic Capacitance .................................... 46

5.4 Extracting and Simulating the Whole Chip Layout .............. 47

VI THE MEASUREMENT OF THE CHIP ..................................... 48

6.1 The Difficulties of High-Frequency Measurements ............ 49

6.2 The Measurement Process …................................................ 50

VII $\quad$ CONCLUSION AND FUTURE WORK ….................................... 57

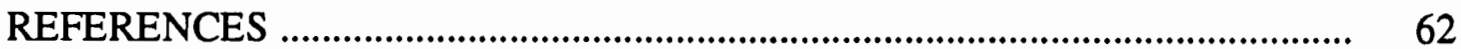

APPENDIXS

I. Post Process MOSIS N14Q SPICE Model ..................................... 65

II. Netlist of Some Circuits _............................................................... 67 


\section{LIST OF TABLES}

$\begin{array}{ll}\text { TABLE } & \text { PAGE }\end{array}$

I Converter Input/Output Voltages at Different Frequencies .............................. 39

II Conventer Input/Output Phases at Different Frequencies ............................... 39 


\section{LIST OF FIGURES}

$\begin{array}{ll}\text { FIGURE PAGE } & \text { PAR }\end{array}$

1 Test circuit for the filter measurement. ...................................... 2

2 A symbol and small-signal AC model of MOSFET. ...................... 5

3 A basic transconductance cell. .................................................... 6

$4 \quad$ Another basic transconductance cell. ............................................... 8

$5 \quad$ 4-transistor transconductance cell. ................................................ 9

6 A differential input/output OTA. .............................................. 11

7 SPICE simulation of DC transfer function of circuit in Fig. 6. ......... 13

8 Frequency response of the differential OTA in Fig. 6. .................... 14

9 Differential input/output OTA 2............................................. 16

10 DC transfer function of OTA 2. ............................................... 18

11 Frequency response of OTA 2 ................................................ 18

12 DC transfer function of OTA 2 with single-ended input. ................. 19

13 Differential-to-single-ended OTA design. ................................... 21

14 Offset-reduced single-ended input/output $G_{m}$ cell. ....................... 22

15 Phase performance of circuits in Fig. 13 and Fig. 5. ........................ 23

16 DC transfer functions of circuits in Fig. 13 and Fig. 5. ................... 23

17 CMOS differential high-speed buffer circuit. ............................... 27

18 DC transfer function of the buffer. .................................................. 29

19 Frequency response of the buffer. ............................................. 30

20 DC performance of the first initial converter design. ..................... 33

21 The principle circuit of the second converter design. ...................... 35

22 The principle circuit of the cascade voltage converter. .................... 37 
23 DC performance of the cascade voltage converter. ......................... 38

24 AC performance of the cascade voltage converter. ......................... 38

25 A layout of a p-well CMOS structure (a) and its top view (b). ........ 43

26 Basic latch loop formed by parasitic transistors and resistors .......... 43

27 The Magic layout of the converter chip. ........................................ 45

28 SPICE simulation (DC) of the converter chip layout. ..................... 48

29 SPICE simulation (AC) of the converter chip layout. ..................... 48

$30 \quad$ The photography of the chip die. ............................................. 51

31 The measurement set-up for the converter chip. ........................... 51

32 The AC measurement at 2nd test board at $f=52 \mathrm{MHz}$.................. 53

33 THe bandwidth measurement results of the chip. ........................ 54

34 DC transfer function measurement result of the chip. .................... 55

35 The circuitry of filter with internal voltage converters. .................... 56

36 The filter measurement (AC) with external voltage transformers. ... 56

37 The filter measurement (AC) with internal voltage converters. ....... 56

38 The measurement (DC) of a single-ended buffer. .......................... 56

39 An improved single-ended buffer. ............................................. 59

40 The DC transfer functions of original and improved buffers. .......... 60 


\section{CHAPTER I}

\section{INTRODUCTION}

In recent years, with the growing industrial demand for signal processing systems operating at higher and higher frequencies, more and more attention is being paid to analog signal processing (ASP) techniques and circuits. Because continuous-time analog circuits do not need switching circuitry, sampling, or A/D and D/A conversion, have lower power consumption, and no switching noise, they have special advantages in applications in many fields, such as filters, communication systems, and read-out and preprocessing electronics in detector arrays.

Operational transconductance amplifiers (OTAs) have simple circuitry, good linearity and very wide bandwidth. Using transconductors and capacitors to build highfrequency circuitry is being given more and more attention, especially for OTA-C integrated filters. This results in the design of a high-performance transconductor becoming an important task.

Transconductance-capacitor filter technology is developing very fast because it provides a way to build continuous-time filters in integrated circuits. The method to build transconductance- $\mathrm{C}$ filters uses one transconductance to simulate a passive resistor, and use two transconductances and one capacitor to simulate a passive inductor.

Some attractive filter designs have been given [1 - 5, 21]. The design in [5] gives a low-pass filter working at very high cut-off frequency $(98 \mathrm{MHz})$. Because the circuitry has a balanced differential input (and differential output) OTA as the basic cell, a purely differential input signal is needed. Since the signal coming from a signal generator is single-ended, in its measurement setup (see Fig. 1), a high-frequency voltage transformer 
was used to convert the single-ended signal to purely differential inputs for the next stage of the filter. In the set-up, another transformer is used to transfer the differential output to a single-ended output which goes to the measurement instruments.

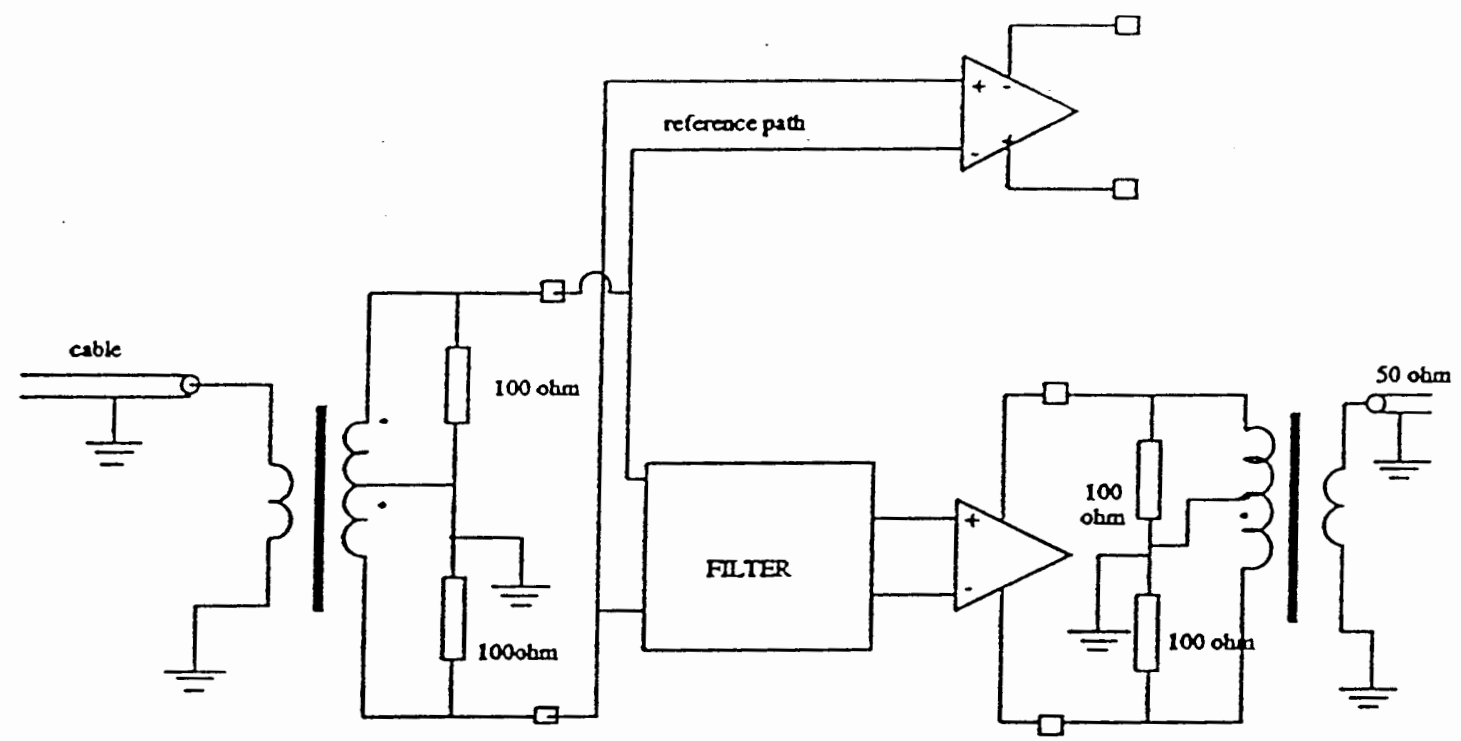

Figure. 1. Test circuit for the filter measurement.

This measurement setup performs well, but it has some problems. One is that the voltage transformer has a huge volume compared to that of integrated filter. If we want to design a fully integrated filter (which usually has differential input and output), the transformer should be integrated into the chip which is measured. Another problem is that the transformer can just work over a finite frequency range, it can not work for DC, low-frequency and very-high-frequency signals. This will limit the use of the filter which is supposed to work over a wide range.

Fully-balanced circuits can cancel out all even-harmonic distortions caused by nonlinearity, and have much better signal-to-noise ( $\mathrm{S} / \mathrm{N}$ ) ratio, power supply rejection ratio (PSRR) and common-mode rejection ratio (CMRR). Therefore, differential input and output designs are often used in OTA-C filters and other high-performance circuits. 
In the real world, the signal source usually is single-ended, and voltage converters (single-ended to differential and differential to single-ended) are needed.

In this project, we use CMOS and OTA techniques to design high-speed voltage converters and some other measurement circuits. First, we study the single-ended to differential voltage converter.

The requirements for the converter are very strict. It should work from DC to $100 \mathrm{MHz}$ or more and the harmonic distortion (nonlinearity) must be small. Its output must give a purely differential signal. Therefore, the converter needs to meet the following requirements:

A. It can work from DC to very high frequencies with a cut-off frequency of at least $100 \mathrm{MHz}$.

B. It has good linearity and low harmonic distortion $(<1 \%)$ up to very high frequencies over a $\pm 0.5 \mathrm{~V}$ input (output) range.

C. Its DC offset is small.

D. The output signals are purely differential, i.e., the two outputs have the same amplitude, opposite phase, and same phase error at any frequency.

E. It has adequate driving ability to drive the next stage, and load.

We used OTAs as basic cells to design the converters. So designing good OTAs is very important. We designed several differential OTAs and an output buffer. Then based on these basic cells, we designed the high-frequency analog voltage converter. The design was simulated by SPICE. A layout of the circuit was created with a layout tool: Magic. Finally, the chip was fabricated. 


\section{CHAPTER II}

\section{OTA DESIGN}

We use OTAs as basic cells to design the voltage converters. First we design differential-input (differential-output) transconductance amplifiers (OTAs). The basic idea is to find first a small basic OTA cell, then use current mirrors to get purely differential output currents. we can use cascode current mirrors to obtain higher output impedance and more accurate current copies.

\subsection{Basic OTA Cells}

Since OTAs find more and more applications in high frequency circuitry, there are many papers introducing different OTA cell designs [6-9]. In our case, we prefer to use some wide-bandwidth, good-linearity, as well as simple-circuitry MOSFET OTAs. First we will study how a MOSFET works.

MOSFET (Metal-Oxide-Silicon Field-Effect-Transistor) is a four-terminal electronic element; its symbol is shown in Fig. 2.1a and its equivalent small-signal model in Fig. 2.1b. Following the general notation, $V_{i j}$ represents the voltage between terminals $i$ and $j$ and $I_{i}$ is used for the current through terminal $i$. Using the square-law model, the drain current, $I_{d}$, of a MOSFET is determined by the voltages $V_{g s}$ and $V_{d s}$, and can be written as

$$
I_{d}=\left\{\begin{array}{lc}
0 & V_{g s} \leq V_{T} \\
k\left[2\left(V_{g s}-V_{T}\right) V_{d s}-V_{d s}^{2}\right]\left(1+\lambda V_{d s}\right) & V_{T} \leq V_{g s} \& V_{d s} \leq V_{d s, s a t} \\
k\left(V_{g s}-V_{T}\right)^{2}\left(1+\lambda V_{d s}\right) & V_{T} \leq V_{g s} \& V_{d s, s a t} \leq V_{d s}
\end{array}\right.
$$


where

$$
k=\frac{\mu C_{o x}}{2} \frac{W}{L}
$$

is called transconductance parameter and $V_{d s, s a t}=V_{g s}-V_{T}$ is the saturation voltage. $\mu$ is the mobility of electrons or holes, $C_{o x}$ is gate oxide capacitance per unit area, $\mathrm{W}$ and $\mathrm{L}$ are the channel width and channel length of the transistors, respectively. $V_{T}$ is the threshold voltage of the transistor and $\lambda$ is the channel-length modulation parameter.

G

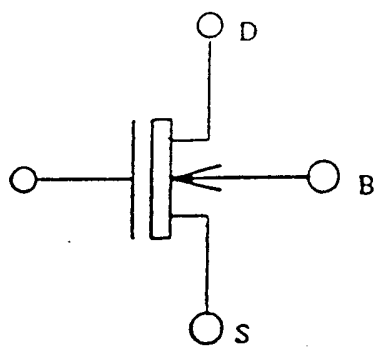

NMOSFET

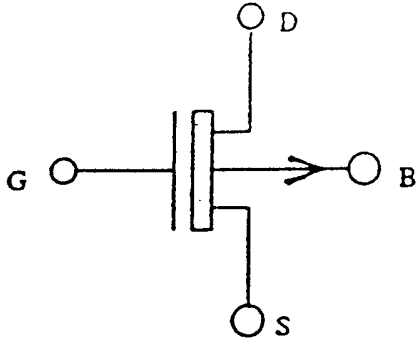

PMOSFET

(a)

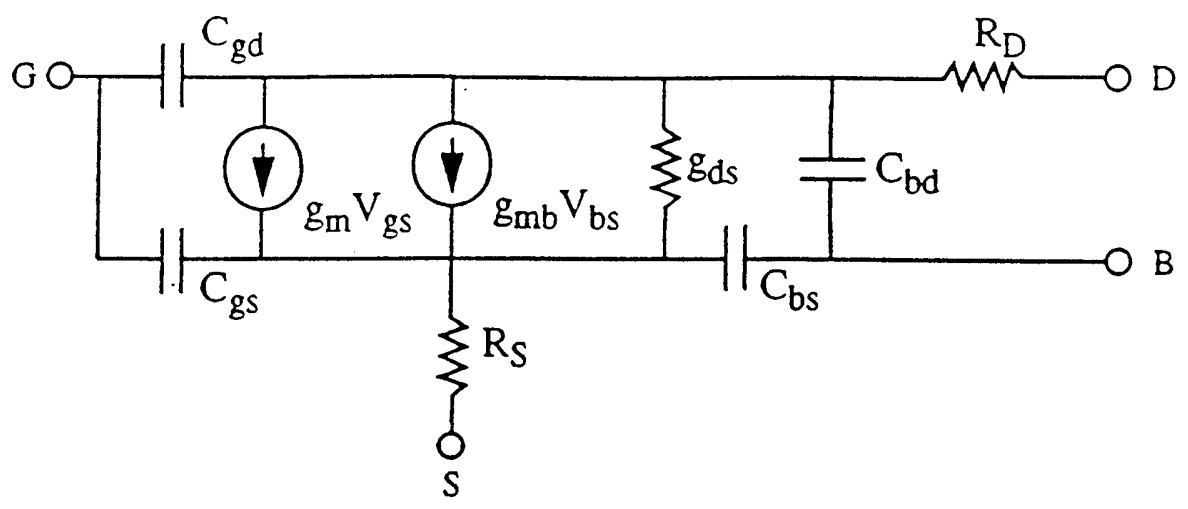

(b)

Figure. 5. A symbol (a) and a small-signal AC model (b) of a MOSFET. "D" represents the drain terminal of the MOSFET, "G" the gate, "S" the source, and "B" the bulk. $C_{g d}$ is the gate-drain capacitor, $C_{g s}$ the gatesource capacitor, $C_{b d}$ the bulk-drain capacitor, and $C_{b s}$ the bulk-source capacitor. $R_{D}$ is the bulk resistor of the drain diffusion, $R_{S}$ the bulk resistor of the source diffusion, and $r_{d s}$ the small-signal output resistor between source-drain. 
In (2.1), the range $\left\{V_{g s} \leq V_{T}\right\}$ is called cut-off region, the range $\left\{V_{T} \leq V_{g s} \& V_{d s} \leq V_{d s, s a t}\right\}$ is the triode region, and the saturation region is in $\left\{V_{T} \leq V_{g s} \& V_{d s, s a t} \leq V_{d s}\right\}$. A MOS transistor is usually biased in the saturation region because: (1) A MOS transistor is easier to bias in the saturation region than in the triode region. (2) In the saturation region $I_{d}$ is large and is almost independent of $V_{d s}$ because $\lambda$ is small. (3) The output impedance, $r_{d s}$, is near constant with a large value, which is

$$
r_{d s}=\left(\frac{\partial V_{d s}}{\partial I_{d}}\right)_{I_{D}}=\frac{1}{\lambda k\left(V_{G S}-V_{T}\right)^{2}}=\frac{1+\lambda V_{D S}}{\lambda I_{D}} \approx \frac{1}{\lambda I_{D}}
$$

In saturation, the transistor can be seen as a voltage-controlled current source, or a transçonductor, with the transconductance being

$$
g_{m}=\left(\frac{\partial I_{d}}{\partial V_{g s}}\right)_{I_{D}}=2 k\left(V_{G S}-V_{T}\right)\left(1+\lambda V_{D S}\right)=\frac{2 I_{D}}{V_{G S}-V_{T}}
$$

neglecting the body effect and channel-length modulation effect, the drain current $I_{d}$ is

$$
I_{d}=k\left(V_{g s}-V_{T}\right)^{2}
$$

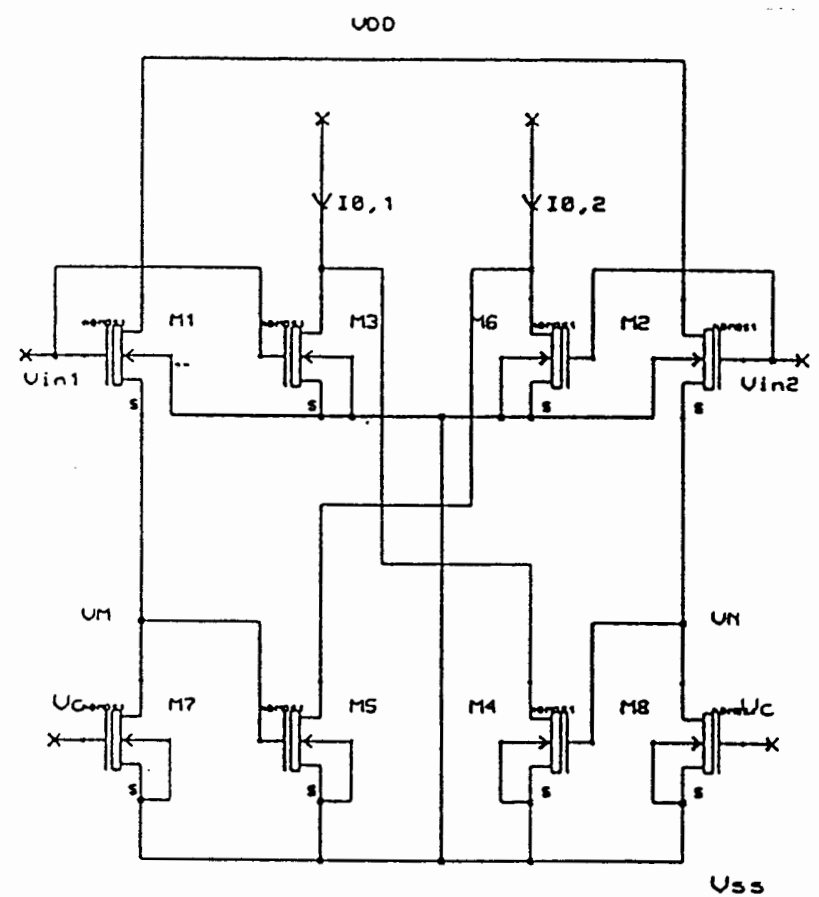

Figure. 3. A basic transconductance cell. 
An OTA design is shown in Fig. 3, where $M_{1}-M_{8}$ compose the basic OTA cell. Assume the drain current through $M_{i}$ is $I_{i}$, all transistors work in the saturation region, and $V_{C}$ is a bias voltage; then

$$
\begin{aligned}
& I_{3}=k_{3}\left(V_{\text {in } 1}-V_{S S}-V_{T}\right)^{2} \\
& I_{4}=k_{4}\left(V_{\text {in } 2}-V_{C}-V_{T}\right)^{2} \\
& I_{5}=k_{5}\left(V_{\text {in } 1}-V_{C}-V_{T}\right)^{2} \\
& I_{6}=k_{6}\left(V_{\text {in } 2}-V_{S S}-V_{T}\right)^{2}
\end{aligned}
$$

where $k_{i}$ is the transconductance parameter defined in (2.2) and $V_{T}$ the threshold voltage. If these transistors have the same size, we have $k_{3}=k_{4}=. .=k$. On deriving eqs. (2.6a) - (2.6d), note that $V_{1}-V_{M}=V_{2}-V_{N}=V_{C}-V_{S S}$, because $M_{1}$ and $M_{7}, M_{2}$ and $M_{8}$ carry the same currents.

By using a current mirrors, the total differential output current $I_{\text {out }}$ can be calculated as a function of the differential input voltage $V_{d}=V_{i n 1}-V_{i n 2}$, i.e.,

$$
\begin{aligned}
I_{\text {out }} & =I_{o, 1}-I_{o, 2} \\
& =I_{3}+I_{4}-I_{5}-I_{6} \\
& =2 k\left(V_{C}-V_{S S}\right) V_{d}
\end{aligned}
$$

Therefore, the basic OTA has a constant transconductance

$$
g_{m}=2 k\left(V_{C}-V_{S S}\right)
$$

which is independent of $V_{T}$ and can be tuned by changing the bias voltage $V_{C}$. Note that for the basic cell all transistors are NMOSFETs which are chosen to be identical for easy control of process tolerances. To maintain all transistors in Fig. 3 in saturation, the differential input voltage must fulfill the condition

$$
\left|V_{d}\right| \leq 2\left(\left|V_{C}\right|-V_{T}\right)
$$

Fig. 4 shows another basic OTA cell. The principle is very similar to the first one. The only difference is that this circuit uses a simpler basic cell, and needs another vol- 
tage supply. Because it uses another voltage source directly instead of using voltage follower to get the bias voltages, this design has better linearity than the first design, but it needs a good voltage source $V_{B}$. In the next section, we will introduce a differential OTA based on this cell.

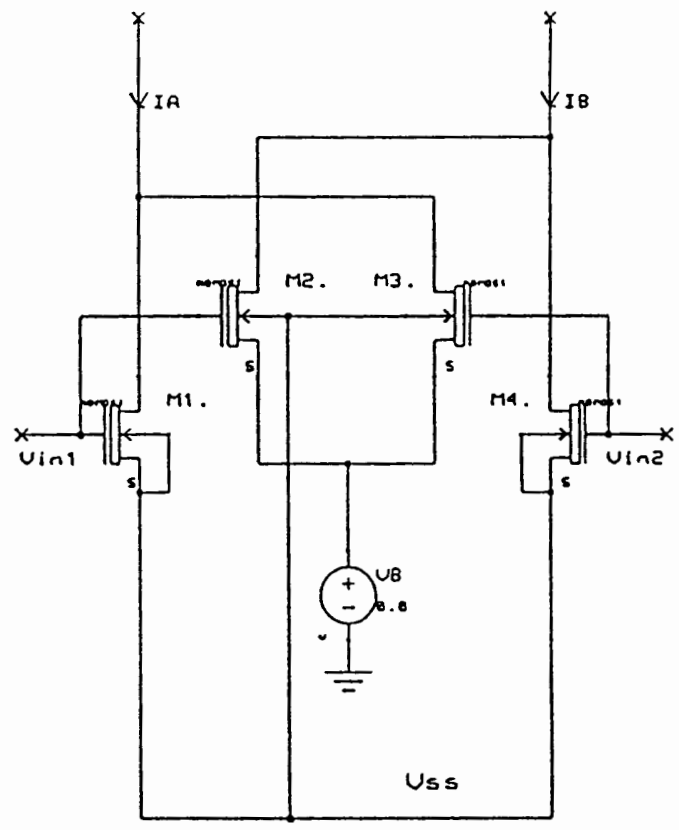

Figure. 4. Another basic transconductance cell.

Another design for a single-ended-input (output) transconductance cell is given by C. S. Park and R. Schaumann (see Fig. 5 and [8]). This transconductance amplifier, $G_{m}$, is a very attractive design, which is very simple (only 4 transistors), and has good linearity and very high bandwidth.

Analyzing this structure is straightforward. Assuming matching between the geometrically identical n-MOS devices, M1, M3, and between the p-MOS devices M2, M4, and using the standard square-law model for MOS devices in their saturation region, the currents $I_{A}$ and $I_{B}$, defined in Fig. 4, are easily derived as

$$
I_{A}=K_{e f f}\left(V_{G 1}-V_{i n}-V_{T n 1}-\left|V_{T p 2}\right|\right)^{2}
$$




$$
I_{B}=K_{e f f}\left(V_{i n}+V_{G 4}-V_{T n 3}-\left|V_{T p 4}\right|\right)^{2}
$$

where

$$
\begin{aligned}
& K_{\text {eff }}=k_{n} k_{p} /\left(\sqrt{k_{n}}+\sqrt{k_{p}}\right)^{2} \\
& k_{n, p}=\frac{1}{2} \mu_{n, p} C_{o x}(W / L)_{n, p}
\end{aligned}
$$

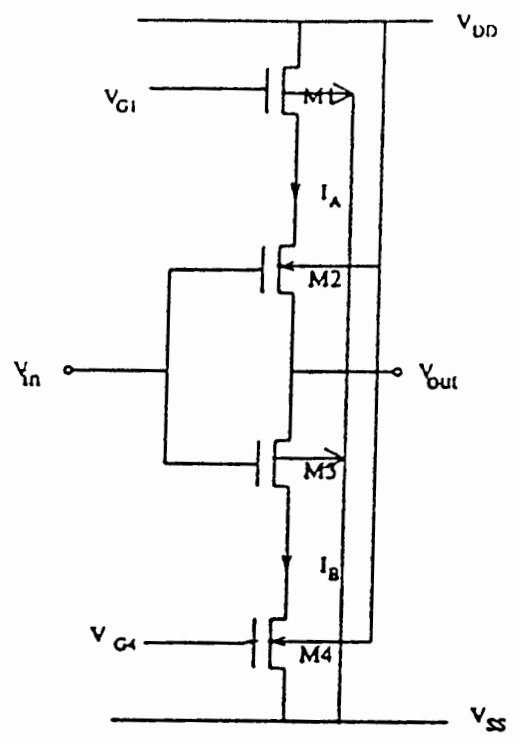

Figure. 5. 4-transistor transconductance cell.

and $V_{T n 1,3}>0, V_{T p 2,4}<0$ are the threshold voltages of the corresponding devices, $V_{G 1}$ and $V_{G 4}$ are bias voltages. For our use, we can assume $V_{T n 1}=V_{T n 3}, V_{T p 2}=V_{T p 4}$. We also connect $V_{G 1}$ to $V_{D D}$ and $V_{G 4}$ to $V_{S S}$, so we do not need additional bias voltages. This yields:

$$
\begin{aligned}
I_{\text {out }} & =I_{A}-I_{B} \\
& =-4 K_{e f f}\left(V_{D D}-V_{T n 1}-\left|V_{T_{p} 2}\right|\right) V_{\text {in }}
\end{aligned}
$$

The $G_{m}$ has a constant transconductance parameter

$$
g_{m}=-4 K_{e f f}\left(V_{D D}-V_{T n, p}\right)
$$

where 


$$
V_{T n, p}=V_{T n, i}+\left|V_{T p, i}\right|
$$

Because OTAs have good characteristics such as high speed, simple circuitry and small silicon area, they are often used to simulate (replace) other basic elements, such as resistors, inductors, etc.

If we connect the input and output of the 4-transistor cell, $G_{m}$, in Fig. 5, we will get a grounded-resistor with a value of $1 / g_{m}$. In integrated circuits, building a resistor takes large area and the accuracy of the resistor value is bad. Additionally, there is a large parasitic capacitance with the resistor so it is rather difficult to build a good resistor (especially a large-value resistor). Using OTAs to replace true resistors is a good way to solve this problem. Of course, there are other ways to build integrated resistors $[10,11]$, but the method mentioned above is very simple, and matches the OTA in performance.

\subsection{Differential Input (Output) OTA Design}

Based on the basic cell in Fig. 4, a differential input (output) OTA was designed (see Fig. 6). It assumes that all $\mathrm{n}$-type transistors have the same parameters and sizes, as do the p-type transistors, for easy control of process tolerances and reduced matching requirements.

Labeling the currents through the eight composite devices $M_{i}, M_{i N}$ and $M_{i}, M_{i} P$ as $I_{i}, i=1, \ldots, 8$, and using eqs. (2.12)-(2.15) with $V_{D D}=-V_{S S}$, yields

$$
\begin{aligned}
I_{1} & =K_{e f f}\left(V_{i n 1}-V_{S S}-V_{T n 1}-V_{T p 1}\right)^{2} \\
& =K_{e f f}\left(V_{i n 1}+V_{D D}-V_{T n p 1}\right)^{2}
\end{aligned}
$$

and

$$
\begin{aligned}
& I_{2}=K_{e f f}\left(V_{i n 1}+V_{B}-V_{T n p 2}\right)^{2} \\
& I_{3}=K_{e f f}\left(V_{i n 2}+V_{B}-V_{T n p 3}\right)^{2}
\end{aligned}
$$




$$
I_{4}=K_{e f f}\left(V_{i n 2}+V_{D D}-V_{T n p 4}\right)^{2}
$$

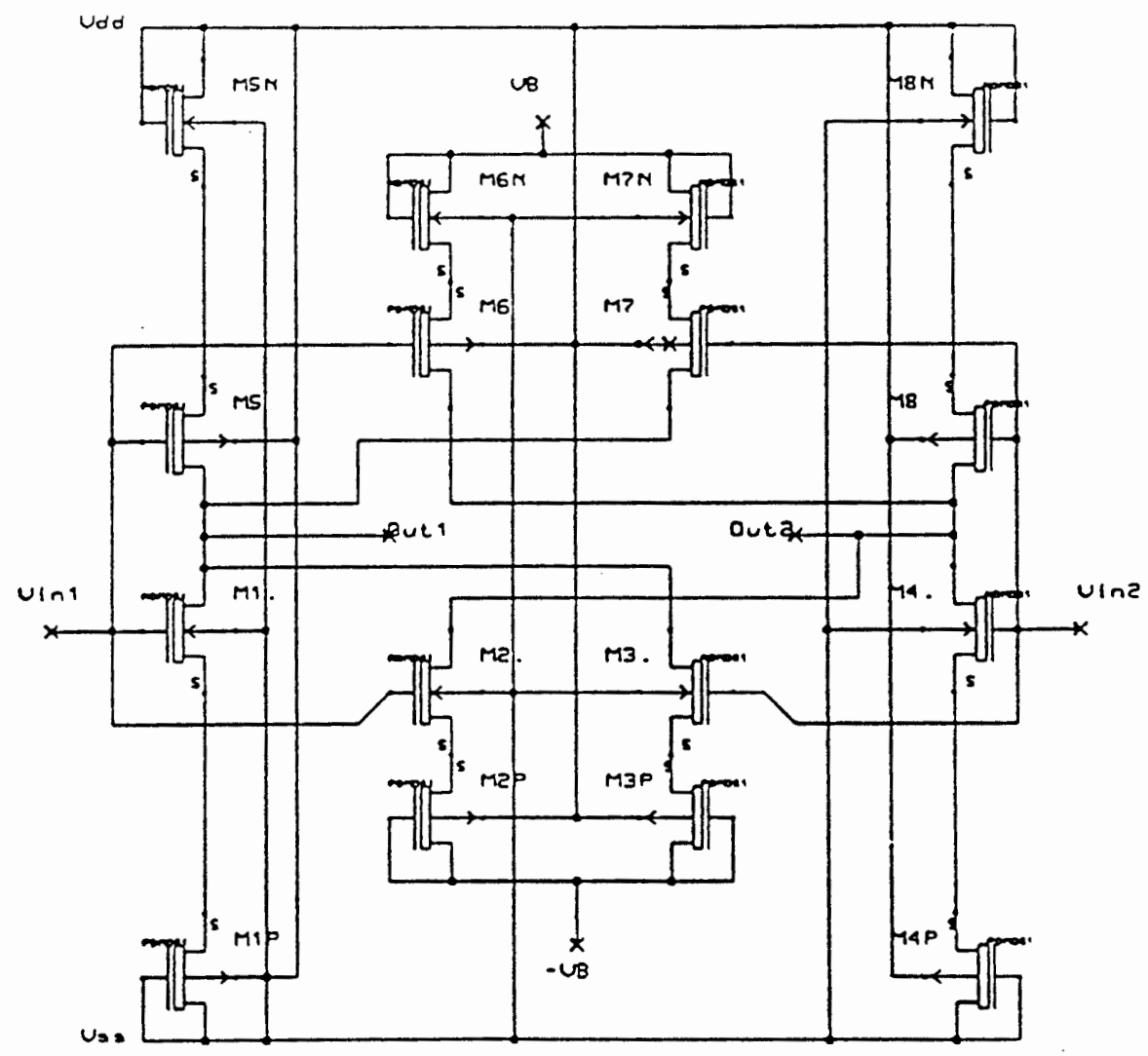

Figure. 6. A differential input/output OTA.

Similarly,

$$
\begin{aligned}
& I_{5}=K_{e f f}\left(V_{D D}-V_{i n 1}-V_{T n p 5}\right)^{2} \\
& I_{6}=K_{e f f}\left(V_{B}-V_{i n 1}-V_{T n p 6}\right)^{2} \\
& I_{7}=K_{e f f}\left(V_{B}-V_{i n 2}-V_{T n p 7}\right)^{2} \\
& I_{8}=K_{e f f}\left(V_{D D}-V_{i n 2}-V_{T n p 8}\right)^{2}
\end{aligned}
$$

The currents through terminals Out 1 and Out2, $I_{\text {out } 1}$ and $I_{\text {out } 2}$, respectively, are

$$
I_{\text {out } 1}=\left[\left(I_{5}+I_{7}\right)-\left(I_{1}+I_{3}\right)\right]
$$




$$
I_{\text {out } 2}=\left[\left(I_{6}+I_{8}\right)-\left(I_{2}+I_{4}\right)\right]
$$

Eq. (2.26), with (2.18)-(2.25), yields

$$
\begin{aligned}
I_{\text {out } 1}= & K_{\text {eff }}\left(2 V_{D D}-V_{T n p 1}-V_{T n p 5}\right)\left(-2 V_{\text {in } 1}-V_{T n p 5}+V_{T n p 1}\right) \\
& +K_{\text {eff }}\left(2 V_{B}-V_{T n p 3}-V_{T n p 7}\right)\left(-2 V_{\text {in } 2}-V_{T n p 7}+V_{T n p 3}\right)
\end{aligned}
$$

For purely differential inputs, $V_{i n 1}=-V_{i n 2}=\frac{1}{2} V_{i n}$,

$$
\begin{aligned}
I_{\text {out } 1}=- & -2 K_{\text {eff }}\left(V_{D D}-V_{B}+\Delta V_{T}\right) V_{\text {in }}+K_{\text {eff }}\left[\left(2 V_{D D}-V_{T n p 1}-V_{T n p 5}\right) \times\right. \\
& \left.\left(V_{T n p 1}-V_{T n p 5}\right)+\left(2 V_{B}-V_{T n p 3}-V_{T n p 7}\right)\left(V_{T n p 3}-V_{T n p} 7\right)\right]
\end{aligned}
$$

where

$$
\Delta V_{T}=V_{T n p 3}+V_{T n p 7}-V_{T n p 1}-V_{T n p 5}
$$

is very small because $V_{T n p 1}+V_{T n p 5} \approx V_{T n p 3}+V_{T n p 7}$. By the same principle

$$
\begin{aligned}
& I_{\text {out } 2}=2 K_{e f f}\left(V_{D D}-V_{B}+\Delta V_{T}\right) V_{\text {in }}+K_{e f f}\left[\left(2 V_{D D}-V_{T n p 4}-V_{T n p 8}\right) \times\right. \\
& \left.\left.\left(V_{T n p 4}-V_{T n p 8}\right)+\left(2 V_{B}-V_{T n p 2}+V_{T n p 6}\right)\right]\left(V_{T n p 2}-V_{T n p 6}\right)\right]
\end{aligned}
$$

Neglecting the body effect, $V_{T n p i}=V_{T n p j}$ and $\Delta V_{T}=0$, eqs. (2.29) and (2.31) show that the circuit has the ideal linear transconductance parameter

$$
g_{m}=2 K_{e f f}\left(V_{D D}-V_{B}\right)
$$

which is independent of $V_{T}$ and can be tuned by changing the bias voltage $V_{B}$. Body effect causes dc offset (the second terms in (2.29) and (2.31)) and small nonlinearities because $\Delta V_{T}$ is signal-dependent. The dc offset is canceled in differential operation.

The circuit's balanced structure with a symmetrical differential output leads to very low offset and high power supply rejection. Also, because identical cells are used for the upper and lower parts, the traditional current mirrors that we used to obtain current subtraction are eliminated: a simpler circuit is obtained and internal nodes are avoided. We can expect the circuit to have very good high-frequency performance. 
$\underline{\text { Simulation results }}$

With $2 \mu \mathrm{m}$ n-well CMOS process models, the circuit in Fig. 6 was simulated by SPICE. Note that $V_{S S}$ is connected to the substrate of all n-type transistors and $V_{D D}$ to that of all p-type transistors. Thus, body effect is considered. The transistor parameters are $V_{T n}=0.858 \mathrm{~V}, K_{n}=66.0 \mu \mathrm{A} / \mathrm{V}^{2}, V_{T p}=-0.889 \mathrm{~V}, K_{p}=19.1 \mu \mathrm{A} / \mathrm{V}^{2} . V_{D D}=-V_{S S}=$ $5 \mathrm{~V}$. A $3 \%$ layout mismatch among these device is assumed for the simulations. The device sizes (in $\mu \mathrm{m})(W / L)_{n}=25 / 2,(W / L)_{p}=18 / 2$ were used. $V_{B}$ can change to vary the transconductance. The power consumption is about $17 \mathrm{~mW}$.

$D C$ performance: For a load of $R=1 k \Omega$, the simulated DC transfer curve and its slope, the transconductance, are shown in Fig. 7 for $V_{B}=4.3 \mathrm{~V}$ and $4.6 \mathrm{~V}$. Good linearity (linearity error $0.4 \%$ ) is obtained over a differential input range as large as $4 V_{p-p}$. The linearity error is defined as

$$
\varepsilon=\frac{I_{\text {out } 1}-I_{\text {out } 2}-g_{m}(0)\left(V_{\text {in } 1}-V_{\text {in } 2}\right)}{g_{m}(0)\left(V_{\text {in } 1}-V_{\text {in } 2}\right)} 100 \%
$$

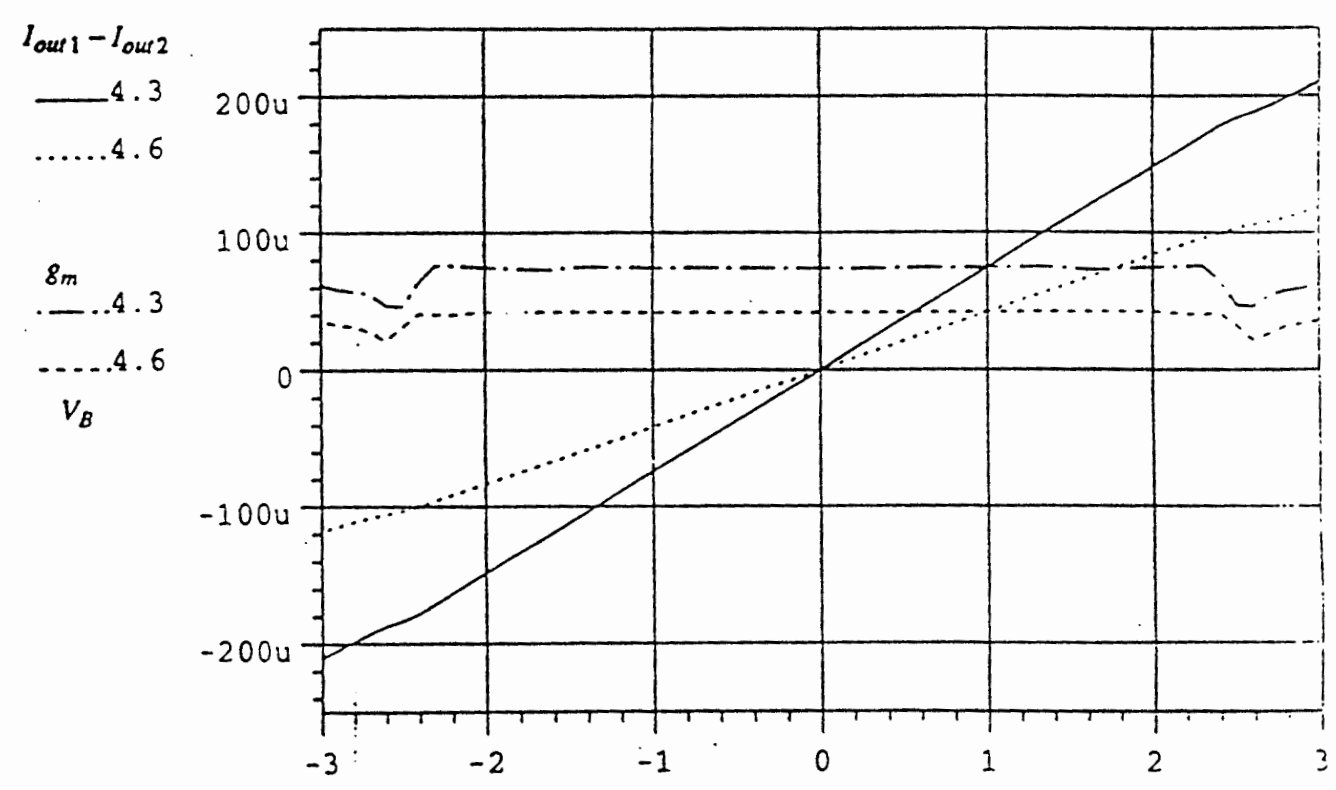

Figure. 7. SPICE simulation of DC transfer function of circuit in Fig. 6. 
where $g_{m}(V)$ is the voltage dependent transconductance. For the assumed layout mismatch of $3 \%$, the offset is very small (about $0.1 \mu \mathrm{A}$ ). Simulations also show that the linearity is only slightly affected by the mismatch of transistor sizes and the mismatch in bias voltages $V_{B},-V_{B}$.

Distortion: For $1 V_{p-p}$ input signal at $f=10 \mathrm{MHz}$ and $100 \mathrm{MHz}$, the simulated harmonic distortion for the circuit is: $\mathrm{THD}=0.19 \%$ at $10 \mathrm{MHz}$ and $\mathrm{THD}=0.26 \%$ at $100 \mathrm{MHz}$.

AC performance: The input capacitance is about $35 \mathrm{fF}$, the output capacitance about $25 \mathrm{fF}$; the output impedance is larger than $100 \mathrm{k} \Omega$ until $100 \mathrm{MHz}$. For a load $R=1 \mathrm{k} \Omega$, paralleled by $\mathrm{C}=50 \mathrm{fF}$ on each output node and $V_{B}=4.3 \mathrm{~V}$, the $-3 \mathrm{~dB}$ frequency of the $G_{m}$ cell reaches $2.2 \mathrm{GHz}$. Note that this frequency is determined by the load $R$ paralleled with $C \approx 75 f F$. The phase error is smaller than 1 degree until $35 \mathrm{MHz}$ (see Fig. 8). The dual-sided power supply rejection (PSR) at $f=10 \mathrm{MHz}$ for both $V_{D D}$ and $V_{S S}$ in $I_{d}=I_{\text {out } 1}-I_{\text {out } 2}$ exceeds $100 \mathrm{~dB}$. Common-mode rejection (CMR) is about $90 \mathrm{~dB}$. The very high cut-off frequency is the most important advantage of the circuit.

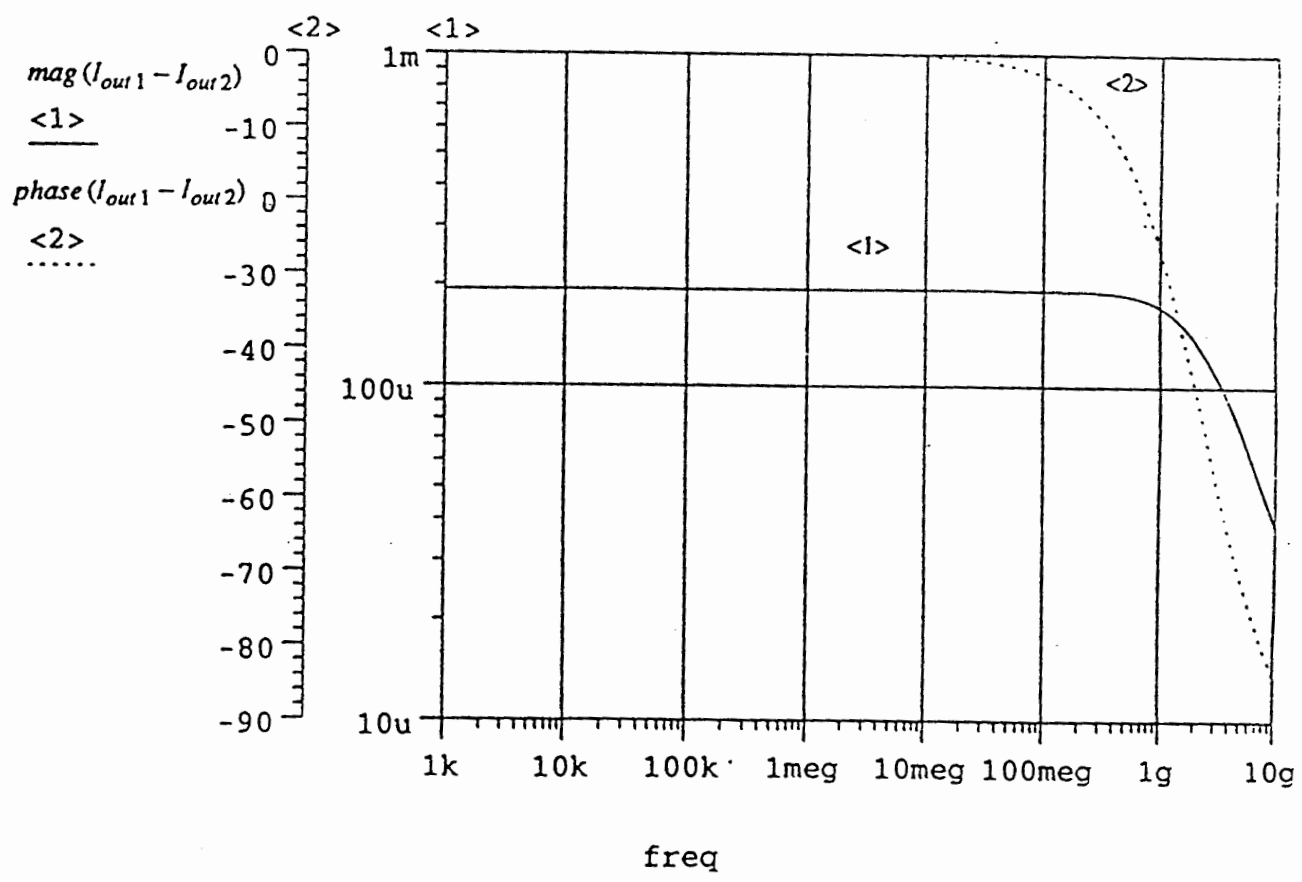

Figure. 8. Frequency response of the differential OTA in Fig. 6. 
The Second Differential OTA

We use the basic OTA cell in Fig. 4 to build another differential OTA (see Fig. 9). Cascode current mirrors are used to create the purely differential current. In Fig. 9, $I_{11}, I_{23}$ and $I_{27}$ are copies of $I_{9}\left(I_{11}=I_{23}=I_{27}=m I_{9}\right) . I_{21}, I_{10}$ and $I_{25}$ are copies of $I_{19}$. At Node Out1,

$$
I_{\text {out } 1}=I_{11}-I_{25}=m\left(I_{9}-I_{19}\right)
$$

where $m$ is the copy ratio. At Node Out2,

$$
I_{\text {out } 2}=I_{21}-I_{27}=m\left(I_{19}-I_{9}\right)
$$

So when $V_{C}=0$, with (2.9)

$$
\begin{aligned}
I_{\text {out } 1} & =m\left(I_{9}-I_{19}\right) \\
& =2 m k\left(V_{C}-V_{S S}\right) V_{d} \\
& =-2 m k V_{S S} V_{d}
\end{aligned}
$$

where $k$ is defined in (2.2); thus

$$
I_{\text {out } 1}=-I_{\text {out } 2}
$$

The circuit is a purely differential output OTA. The input can be purely differential or a single-ended, but it has much better performance for differential input.

Note that we use cascode current mirrors instead of using simple current mirrors to obtain more accurate current copies and to reduce the errors when transferring the basic OTA currents to the output stage. Another advantage is that cascode current mirrors can provide much higher output impedance for the output stages, as required for a good OTA. 


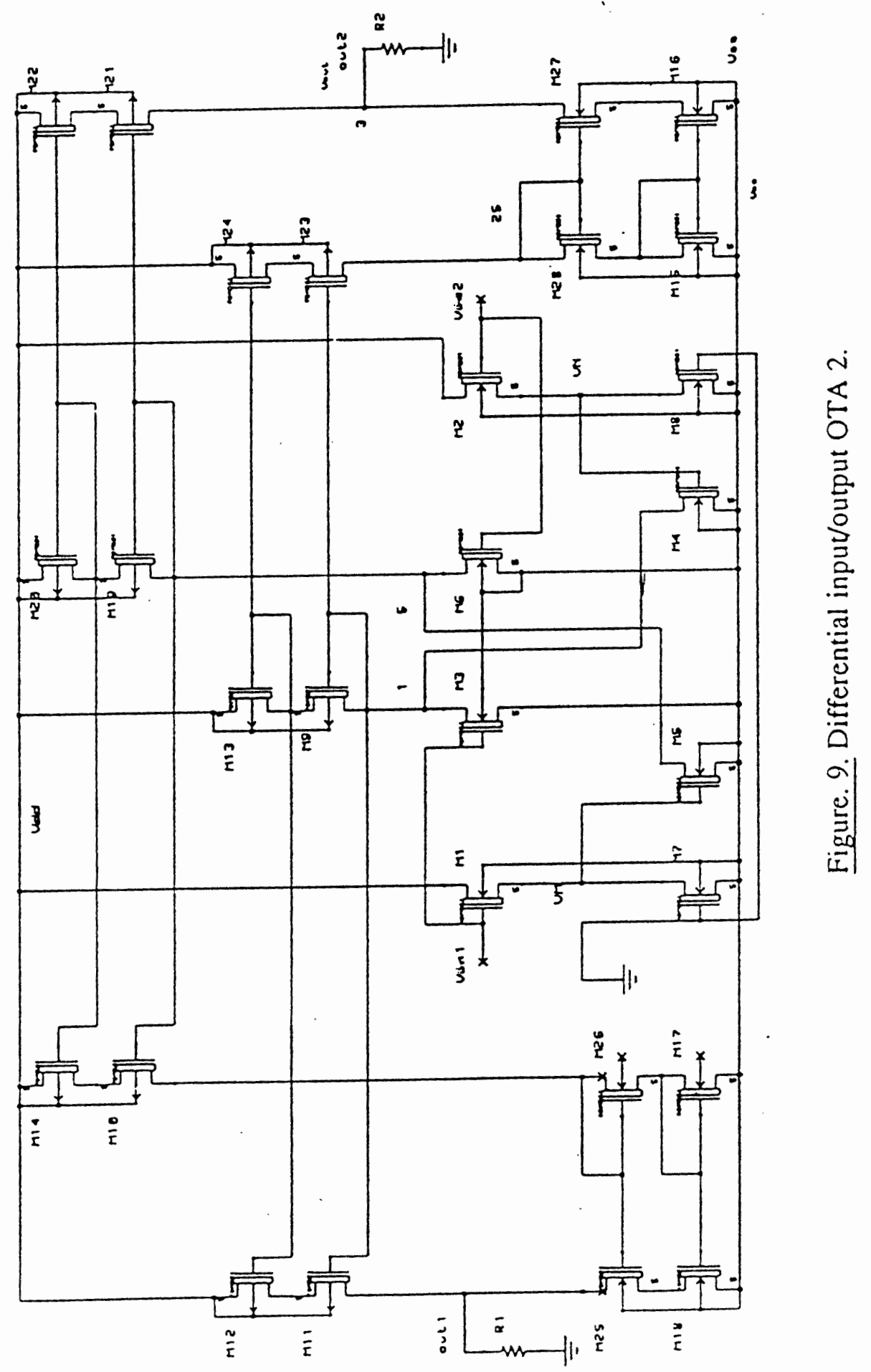


When designing this OTA, we must be very careful choosing the sizes of individual transistors, especially for the current mirrors. Generally speaking, using current mirrors drops the frequency response dramatically. We can analyze the reason for the effect on the frequency response of $I_{\text {out } 1}$.

For an input signal $V_{\text {in }}$, the voltage transfer path is $V_{\text {in } 1} \rightarrow>$ Node $1 \rightarrow$ Node $25->$ $V_{\text {out } 2}$. Because the bandwidth of PMOS transistors is much worse than NMOS, the design for PMOS current mirrors is more important and the pole at Node 1 becomes very critical.

At Node 1, we find the large capacitance

$$
C=\frac{1}{2}\left(C_{g s 9}+C_{g s 23}+C_{g s 11}+C_{g d 9}+C_{g d 23}+C_{g d 11}+C_{g b 9}+\ldots\right)
$$

Usually $C_{g s 9}>C_{g d 9} \gg C_{g b 9}$. If at this node, the impedance is also large, the pole becomes very critical. By choosing the ratio $m$ much smaller than unity, that is, we choose the size of $M_{9}\left(M_{19}\right)$ much larger than $M_{23}, M_{11}\left(M_{10}, M_{21}\right)$, the resistance is very low (about $2 / g_{m} 9$ ). The capacitance is small also because we choose small sizes for $M_{10}, M_{11}$. Thus, the frequency response is improved greatly. Also, we reduce the output current and the whole OTA uses less power. But, at the same time the current gain is reduced.

The performance of the OTA

Based on a MOSIS $2 \mu \mathrm{m}$ CMOS process, we used SPICE to simulate the circuit. The process model is the same as the one we used in the last section. At output nodes Out1 and Out2, a $5 k \Omega$ resistor and a $50 f F$ capacitor were connected as a load (in Fig. 9, the currents through the load resistors $R_{1}$ and $R_{2}$ are $I_{\text {out } 1}, I_{\text {out } 2}$ ). For a purely differential input, the DC and AC performance of the OTA are shown in Fig. 10 and 11. Fig. 11 shows the AC performance with different capacitive loads $(0-0.25 p F)$. 


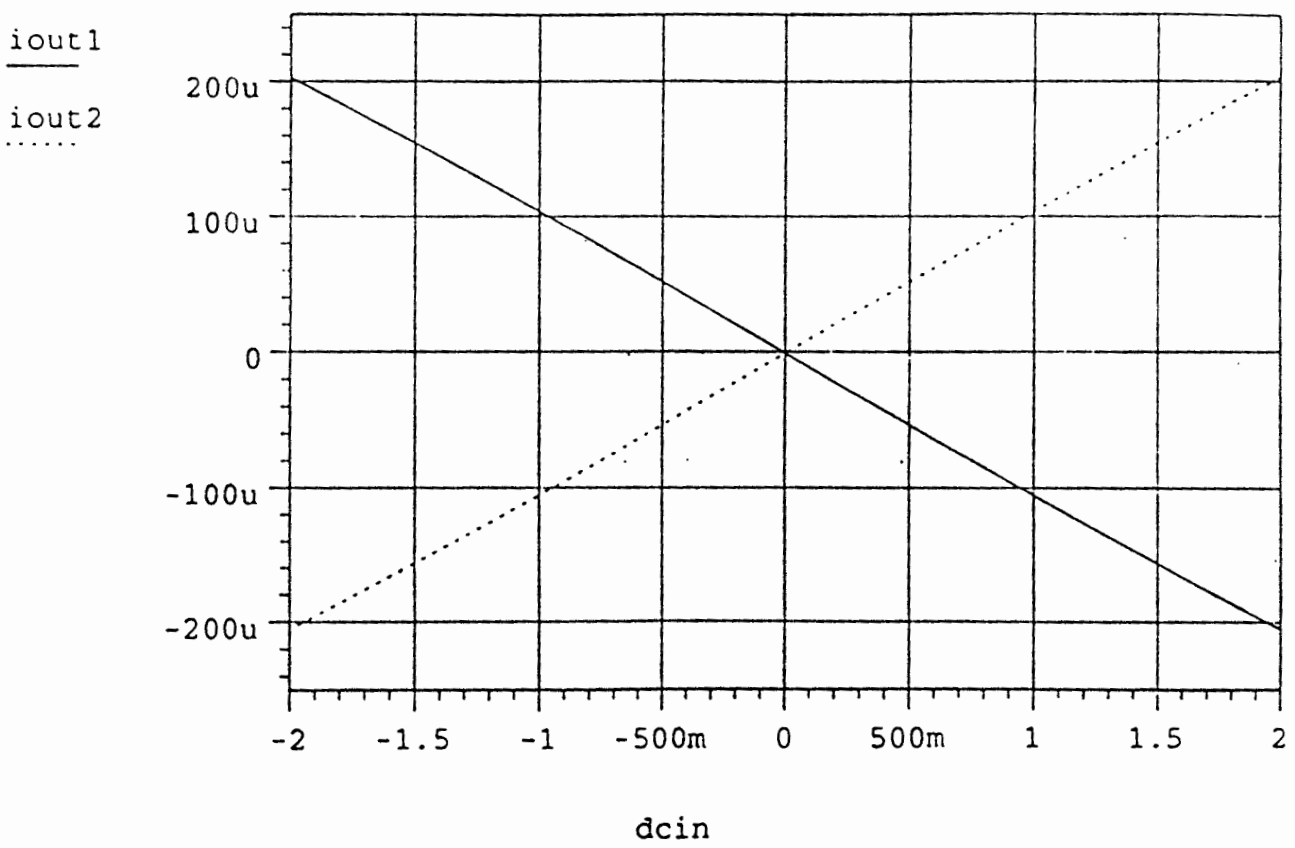

Figure. 10. DC transfer function of OTA 2.

lout 1, iout2 are the two output currents of the OTA.

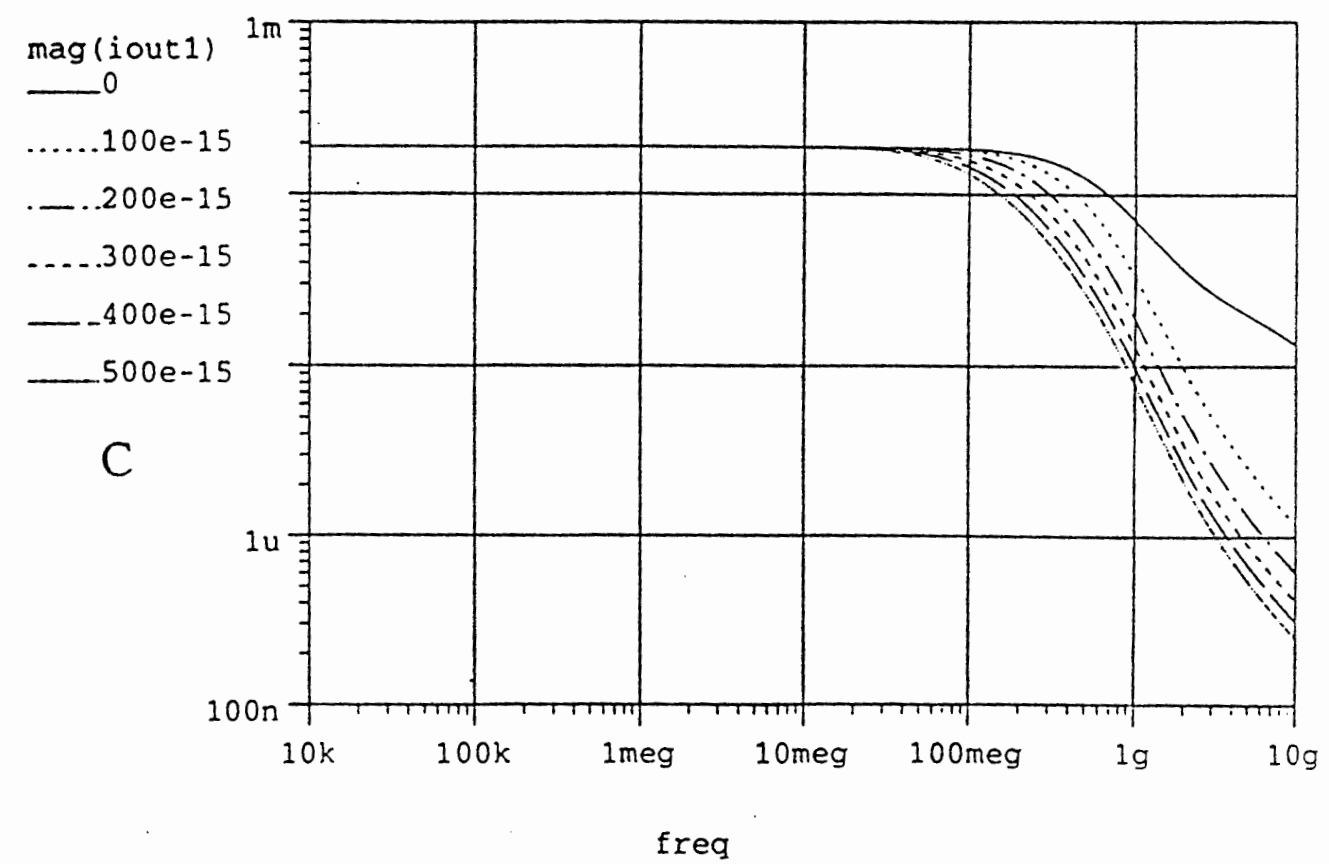

Figure. 11. Frequency response of OTA 2. 
We can see that for an input range of $V_{d}= \pm 2 \mathrm{~V}$ the OTA output current maintains good linearity (error $<0.5 \%$ ). The DC offset is very small (about $1 \mu \mathrm{A}$ ). Also $I_{\text {out } 1}$ and $I_{\text {out } 2}$ (the currents through two load resistors $R_{1}, R_{2}$ ) have the same offset. This results in common-mode offset cancellation in the next stage. For a single-ended input, the DC performance of the OTA is shown in Fig. 12. Compared to the differential input, the linearity is seen to be much worse, because the whole circuitry is symmetric, and some nonlinearities on one side are canceled on the other side. If the input is not differential, we will not have this canceling effect.

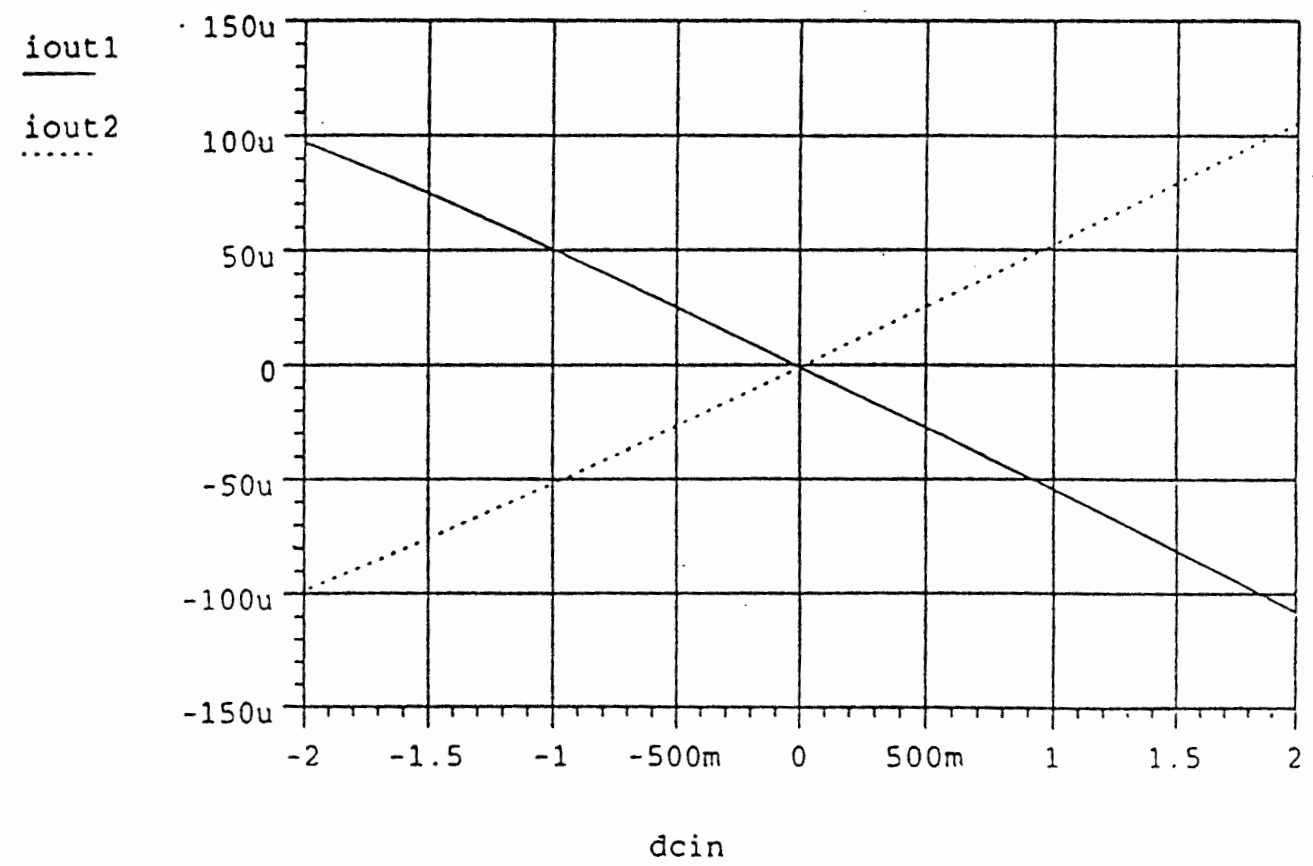

Figure. 12. DC transfer function of OTA 2 with single-ended input. iout1, iout2 are the two output currents of the OTA.

In Fig. 11 the DC response shows that the output is purely differential. With a load of $1 k \Omega$ and $50 f F$, the cut-off frequency $\left(f_{-3 d B}\right)$ can reach $300 \mathrm{MHz}$. Because of the properties of OTAs, such as current output instead of voltage output, low gain stage, etc., the frequency response is very sensitive to the load (especially for a capacitive load). If the OTA needs to drive a large capacitance, an analog buffer is needed, otherwise the 
cut-off frequency will drop dramatically. We will discuss the design of a buffer in the next chapter.

An advantage of this OTA is that besides the power supply $V_{D D}, V_{S S}$ (and Ground), this OTA does not need any other bias voltage.

To obtain good linearity and low offset for this OTA, good current copying is necessary. For accurate current copying, careful attention must be paid to the current mirrors. We already mentioned cascode current mirrors, also we prefer to use large size transistors for this OTA which will give larger transconductance parameter and raise the cut-off frequency. As we discussed in Section 2.1 for the second differential OTA, a large transconductance parameter leads to a lower impedance level and smaller RC time-constant at internal nodes.

\subsection{Differential to Single-ended Output OTA Design}

We discussed the design of a differential input (output) OTA above. Sometimes we need to convert a differential voltage to a single-ended voltage, e.g., for measurements, because an instruments' inputs are single-ended. Compared to single-ended to differential conversion, this is an easier task.

The circuit is shown in Fig. 13. The design principle is similar to the last OTA design (see Fig. 9). We can see from Fig. 13, the same basic OTA cell, and cascode current mirrors are used. Instead of using current mirrors to get two outputs, here we need to get one output. We can see that the circuitry is simpler, and we can expect that the capacitance at the critical nodes is smaller and higher cut-off frequency can be obtained. 


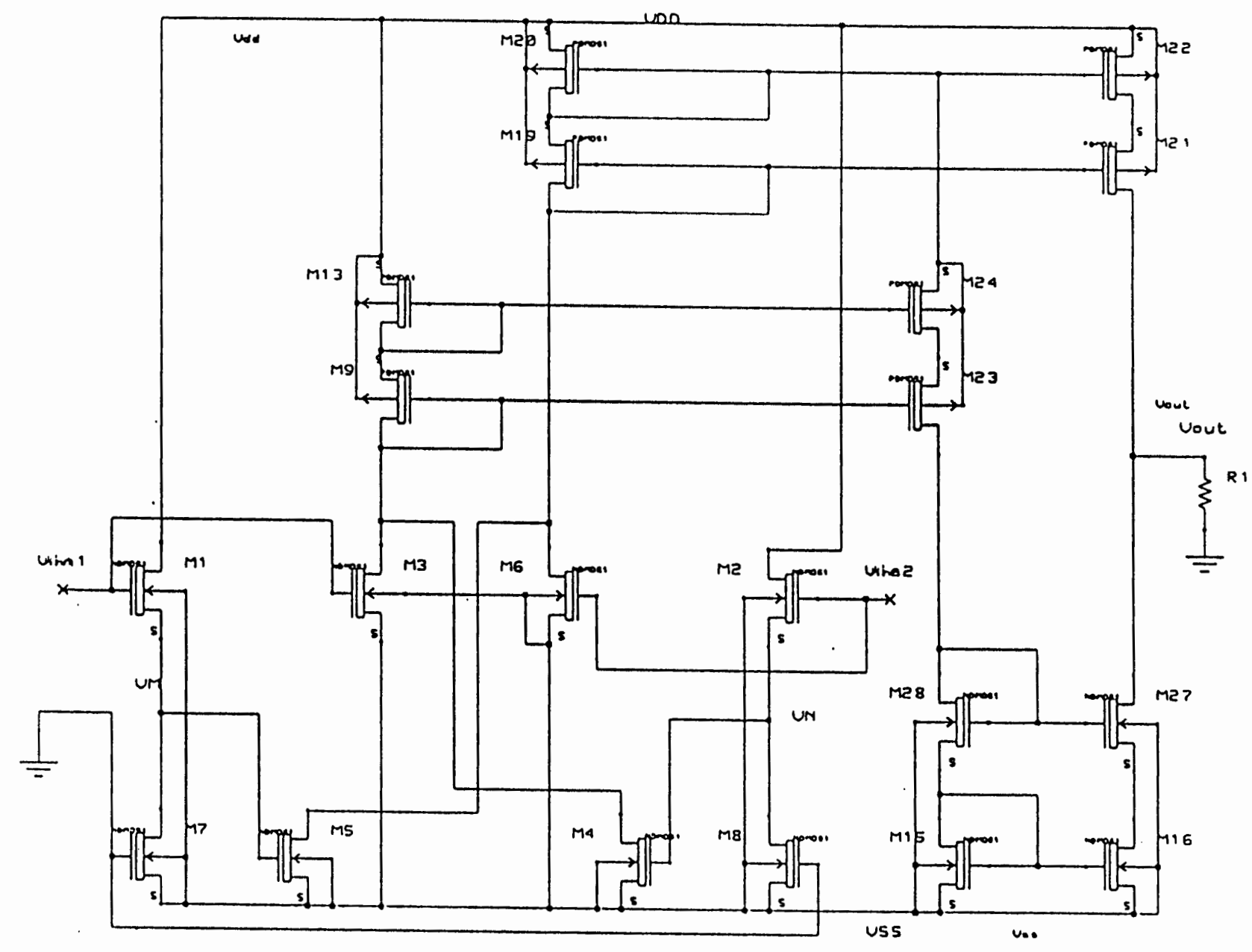

Figure. 13. Differential-to-single-ended OTA design. 


\section{$\underline{2.4 \text { Offset-reduced OTA Design }}$}

The 4-transistor transconductance cell design in Fig. 5 is very simple, but it has some drawbacks. One is that it has a quite large DC offset. In most CMOS processes the substrate of all n-type transistors connects with $V_{S S}$, and the substrate of all p-type transistors connects with $V_{D D}$. This causes body effect and a rather large DC offset for this circuit. Another problem is that its input range is small, about $\pm V_{T}$ because we need to keep all transistors working in the saturation region.

In Fig. 14 we give a method for deleting the DC offset of the transconductance. The principle is that a second transconductance, an exact copy of the first one, is used. The input of the second cell is connected to ground. These two identical cells should have the same DC offset. We use two pairs (n-type and p-type) of current mirrors to transfer the offset of the second cell to the output of the first cell, to delete the offset of the first one. This results in an transconductance with very small DC offset.

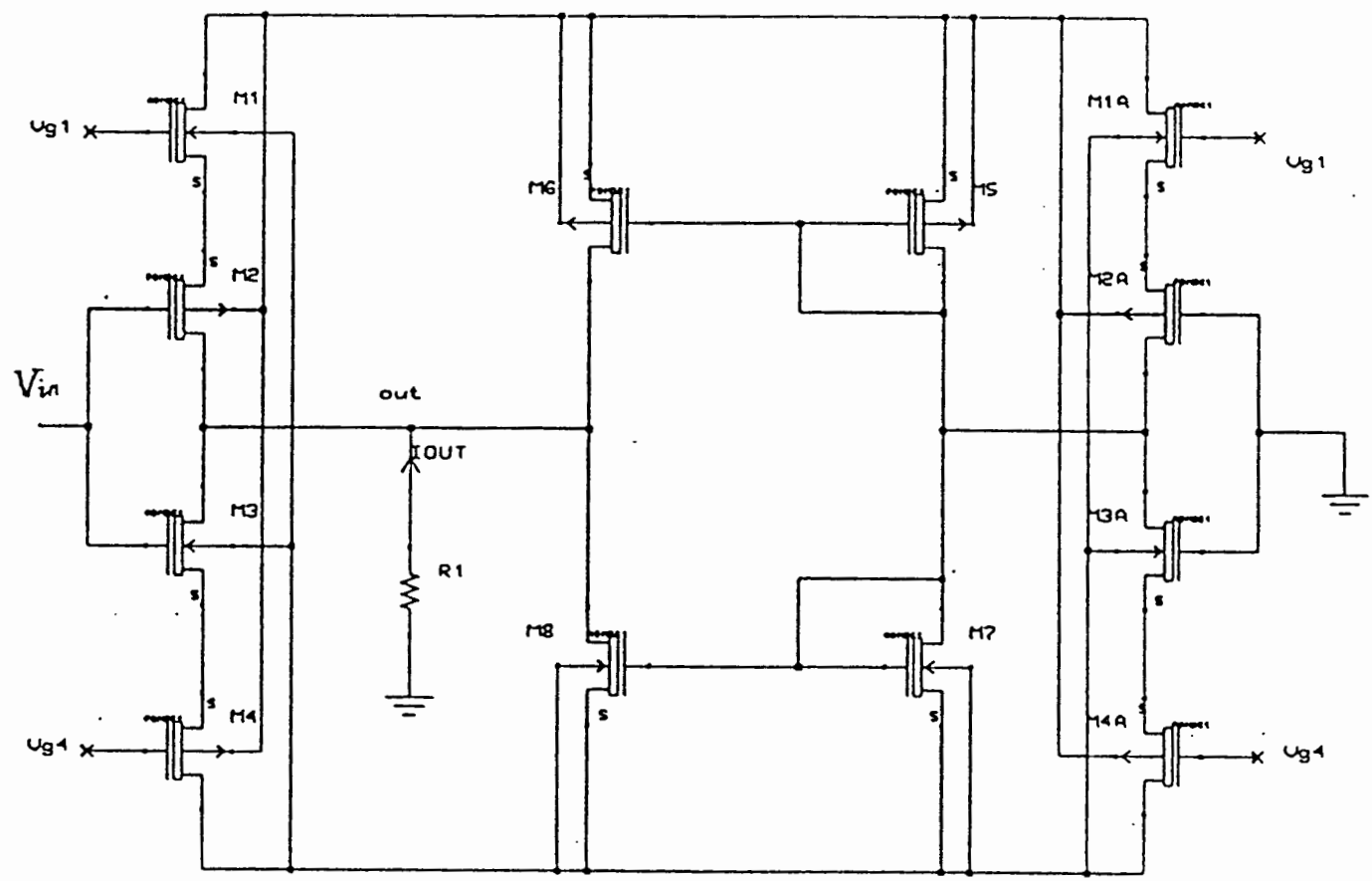

Figure. 14. Offset-reduced single-ended $G_{m}$ cell. 


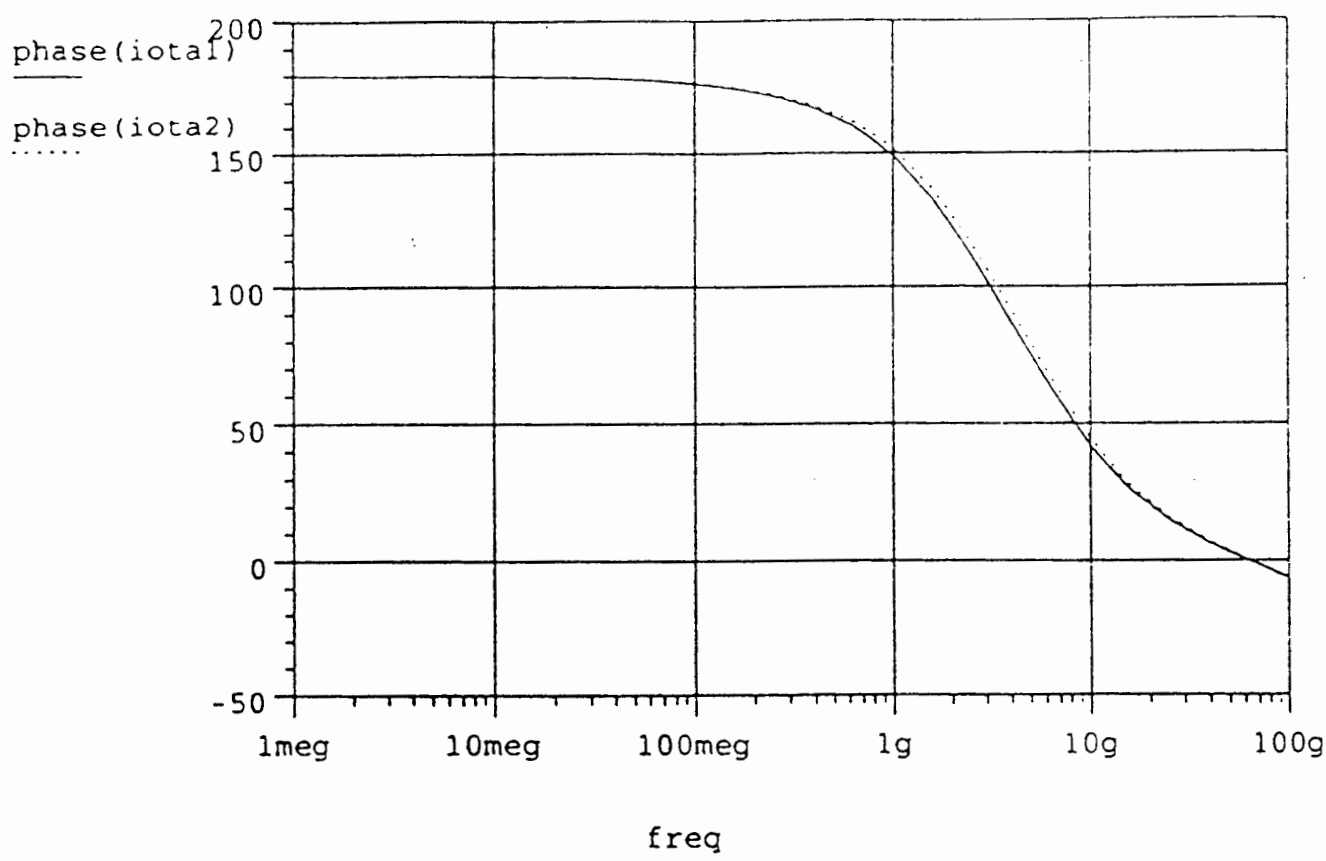

Figure. 15. Phase performance of circuits in Fig. 14 and Fig. 5. iotal is output current of circuit in Fig. 14. iota2 is output current of circuit in Fig. 5.

iota 1

iota 2

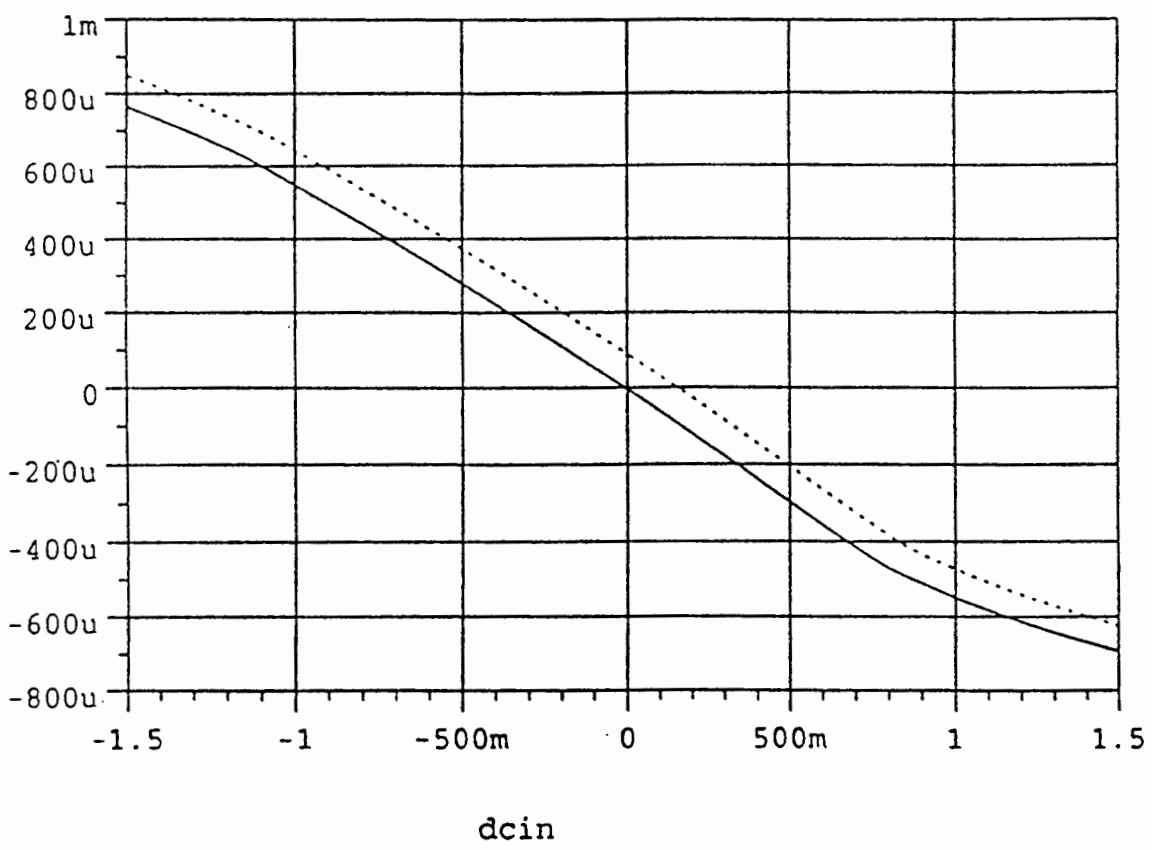

Figure. 16. DC transfer functions of circuits in Fig. 14 and Fig. 5. iotal is output current of circuit in Fig. 14. iout2 is output current of circuit in Fig. 5. 
Because of the body effect, the upper composite transistor (discussed in Section 2.1) has larger transconductance than the lower composite transistor. We can choose wider transistors for the lower device to delete the imbalance. As a result, the linear input range is also extended. The phase and DC performance of this offset-reduced circuit and the original one are shown in Fig. 15 and Fig. 16. A $5 k \Omega$ resistor and a $50 f F$ capacitor were connected as a load. We can see the additional offset-reduced device does not significantly affect the bandwidth of the original device. 


\section{CHAPTER III}

\section{BUFFER DESIGN}

\subsection{Introduction}

In the last chapter, we discussed that the load driving ability of OTAs is often very weak. In many cases a good analog buffer is needed. The requirements for this buffer are:

1. Operating range from $\mathrm{DC}$ to very high frequencies $(>250 \mathrm{MHz})$.

2. Very low harmonic distortion.

3. Low DC offset.

4. Simple circuitry and low power consumption.

These specifications can be met by the differential buffer to be introduced. This circuit can also be used as output buffer for the voltage converter.

Linear high-speed buffers are very useful for both testing and internal signal transfer in integrated circuits (ICs). Although testing of ICs is fairly mature in the digital domain, the testability of the analog portion of a mixed-mode IC is currently a major design problem. The problem is particularly serious in OTA-C integrated filter circuits where differential inputs and outputs are normally used and the working frequency can reach $100 \mathrm{MHz}$ or more [5]. The performance of the ICs (e.g. accuracy, noise, PSRR, CMRR) depends heavily on the amount of parasitic capacitance connected to critical nodes: in some cases even an extra 50fF can result in significant performance degradation. If a circuit needs to drive a following stage with larger input capacitance, a good analog differential buffer is needed. 
Currently most analog buffers appear to work only below $20 \mathrm{MHz}$ and for many applications their linearity is not adequate [12-14]. The shortcomings are addressed by the simple high-speed CMOS differential buffer with excellent linearity presented in the following.

\subsection{Circuit Description}

Consider the circuit in Fig. 17. For a $\pm 5 \mathrm{~V}$ power supply, $M_{9}-M_{12}$ as a bias circuit provides a rather accurate $V_{C 1}=-2.5 \mathrm{~V}$ and $V_{C 2}=+2.5 \mathrm{~V}(-1.25 \mathrm{~V}$ and $+1.25 \mathrm{~V}$ when the power supply is $\pm 2.5 \mathrm{~V}$ ) bias voltage. $M_{1}-M_{8}$ and $M_{1 A}-M_{8 A}$ form the main parts of the buffer. Only the part $M_{1}-M_{8}$ is analyzed because the working principle for $M_{1}-M_{8}$ is the same as that of $M_{1 A}-M_{8 A}$.

Assume that the parameters for all $\mathrm{n}$-channel (and p-channel) transistors are the same. $M_{1}$ and $M_{2}\left(M_{3}\right.$ and $\left.M_{4}\right)$ form an n-channel (p-channel) voltage follower. When all are in saturation, the current through transistor $M_{i}$ is

$$
I_{i}=K_{i}\left(V_{g s i}-V_{T i}\right)^{2}\left(1+\lambda V_{d s i}\right), i=1,2,3,4
$$

where

$$
K_{i}=\frac{\mu C_{o x}}{2} \frac{W_{i}}{L_{i}}
$$

All parameters have their usual meaning as defined in Section 2.1 and $\lambda$ is an empirical channel length modulation factor. Neglecting initially the body effect and channel length modulation,

$$
I_{i}=K_{i}\left(V_{g s i}-V_{T 0}\right)^{2}
$$

Since $I_{1}=I_{2}, I_{3}=I_{4}$ and $V_{G 2}$ of $M_{2}$ is $-2.5 \mathrm{~V}, V_{G 3}$ of $M_{3}$ is $+2.5 \mathrm{~V}$, it follows from (3.3) that $V_{G S 1}=V_{G S 2}=V_{G S 3}=V_{G S 4}=2.5 \mathrm{~V}$, i.e.

$$
V_{1}=V_{\text {in } 1}-2.5 \mathrm{~V}, \text { and } V_{2}=V_{\text {in } 1}+2.5 \mathrm{~V}
$$




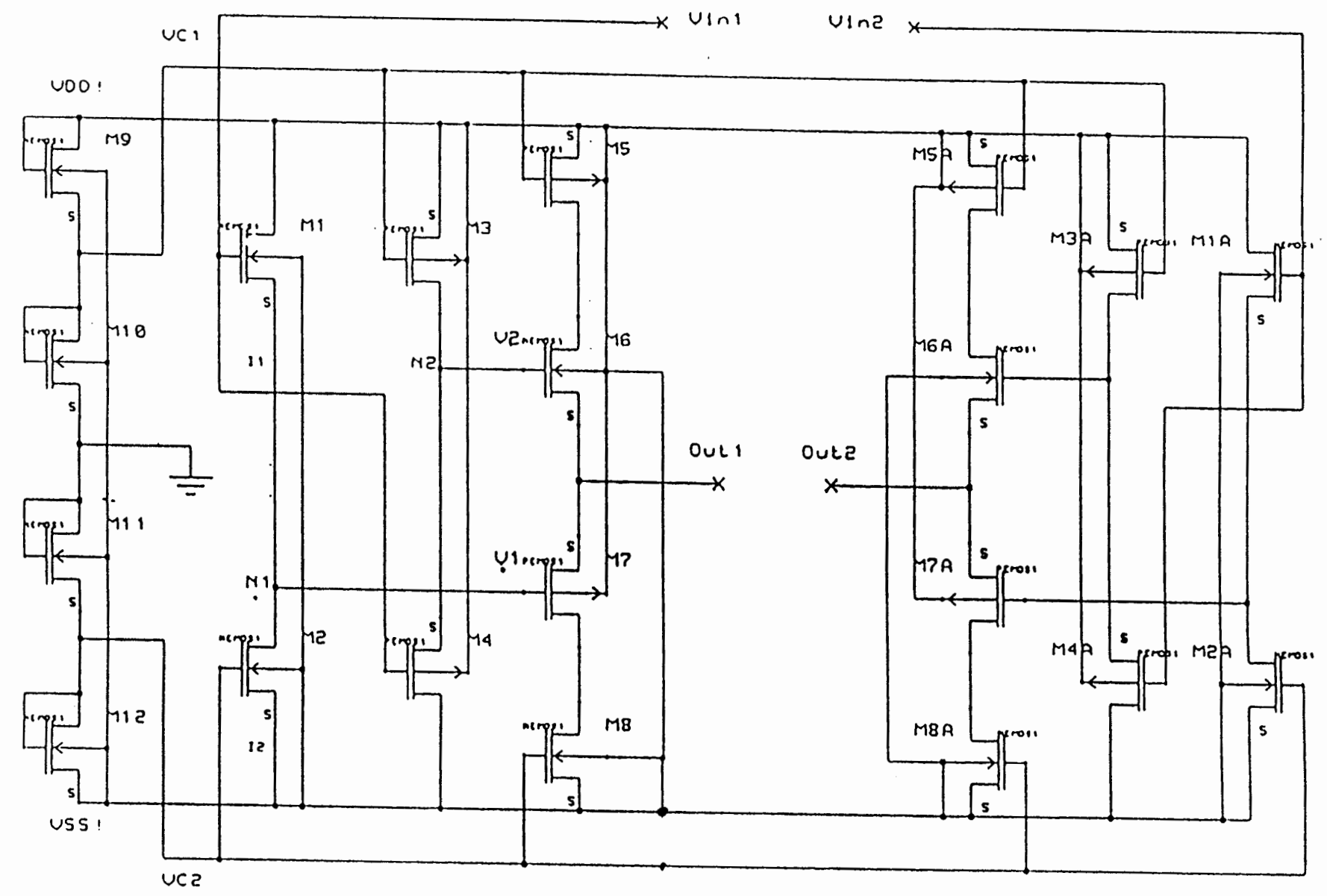

Figure. 17. CMOS differential high-speed buffer circuit. 
As an output stage, $M_{5}, M_{6}$ and $M_{7}, M_{8}$ are two identical "composite transistors" [8] forming a voltage divider. With no input signal $\left(V_{i n 1}=0\right)$, without considering body effect, $V_{1}=-2.5 \mathrm{~V}, V_{2}=+2.5 \mathrm{~V}$, and the voltage of the output node must be at the midpoint between $V_{D D}$ and $V_{S S}$, i.e. $V_{o u t ~}=0$, and $V_{g s 6}=V_{g s 8}=2.5 \mathrm{~V}$. This means that the circuit will delete DC offset.

When $V_{\text {in } 1} \neq 0$, because $M_{5}$ works as a current source and $M_{6}$ is another nchannel source follower, $V_{\text {out } 1}$ must track $V_{2}$, i.e.

$$
V_{\text {out } 1}=V_{2}-V_{g s 6}=\left(V_{\text {in } 1}+2.5 \mathrm{~V}\right)-2.5 \mathrm{~V}=V_{\text {in } 1}
$$

Similarly, for the lower branch $M_{7}, M_{8}$,

$$
V_{\text {out } 1}=V_{1}+2.5 V=\left(V_{\text {in }}-2.5 V\right)+2.5 V=V_{\text {in } 1}
$$

The only way that $V_{\text {out } 1}$ can meet the requirements of both n-channel and p-channel voltage followers is for $V_{\text {out } 1}$ to track $V_{\text {in } 1}$. This is the main reason why the buffer can maintain good linearity and very small offset.

$M_{1 A}-M_{8 A}$ work in the same way as $M_{1}-M_{8}$. Because the characteristics of pchannel transistors are inferior to those of the n-channel devices, the characteristics of the buffer (gain, frequency response, etc.) are determined by the p-path $\left(M_{3}, M_{4}\right.$, and the composite device $M_{5}, M_{6}$ ). The output impedance for this buffer can be approximated as $2 /\left(g_{m 6}+g_{m 7}\right)$. For the circuit in Fig. 3.1 with device sizes identified below, it is of the order of $600 \Omega$. Large-size output transistors help reduce the output impedance in a trade-off for larger area and power consumption. For a load $R_{L}$, assuming $g_{m 3}=g_{m 4} \gg g_{d s 3}=g_{d s 4}$, the gain can be expressed approximately as

$$
A_{v} \approx \frac{g_{m 4}}{2 g_{d s} 3+g_{m 4}} \frac{R_{L}}{R_{L}+1 /\left(g_{m 6}+g_{m 7}\right)}
$$

The body effect in a real process results in a small offset; it can be reduced by using different size transistors for $M_{5}$ and $M_{7}$. 
The buffer has two internal nodes, $\mathrm{N} 1$ and $\mathrm{N} 2$, but the frequency performance is hardly degraded because as sources of voltage followers, both are low-impedance nodes. The same argument holds for the second stage, through which signals flow to the output. Thus, the frequency response for this buffer is excellent.

\subsection{Simulation Results}

The proposed CMOS buffer has been simulated with SPICE. For a standard $2 \mu \mathrm{m}$ n-well CMOS process, the most relevant parameters are: $V_{T n 0}=0.858 \mathrm{~V}, V_{T p 0}=$ $-0.889 \mathrm{~V}, K_{n}=50.5 \mu \mathrm{A} / V^{2}, K_{p}=19.1 \mu \mathrm{A} / V^{2}$. The following device sizes (in $\mu \mathrm{m}$ ) were used: $\quad(W / L)_{1,2}=10 / 2, \quad(W / L)_{3,4}=30 / 2, \quad(W / L)_{5}=60 / 2, \quad(W / L)_{6}=80 / 2$, $(W / L)_{7}=120 / 2, \quad(W / L)_{8}=80 / 2, \quad(W / L)_{9,11}=20 / 20, \quad(W / L)_{10}=17 / 20, \quad(W / L)_{12}=16 / 20$. $V_{D D}$ is connected to the substrates of all p-channel transistors and $V_{S S}$ to those of all nchannel transistors to consider body effect. DC Performance: For $V_{D D}=-V_{S S}=5 V$ and a load of $\mathrm{R}=50 \mathrm{k} \Omega$ paralleled by a $1 p F$ capacitor, the output voltage and the DC gain vs. input voltage are shown in Fig. 18. Note that the gain is smaller than unity because of eq. (3.7).

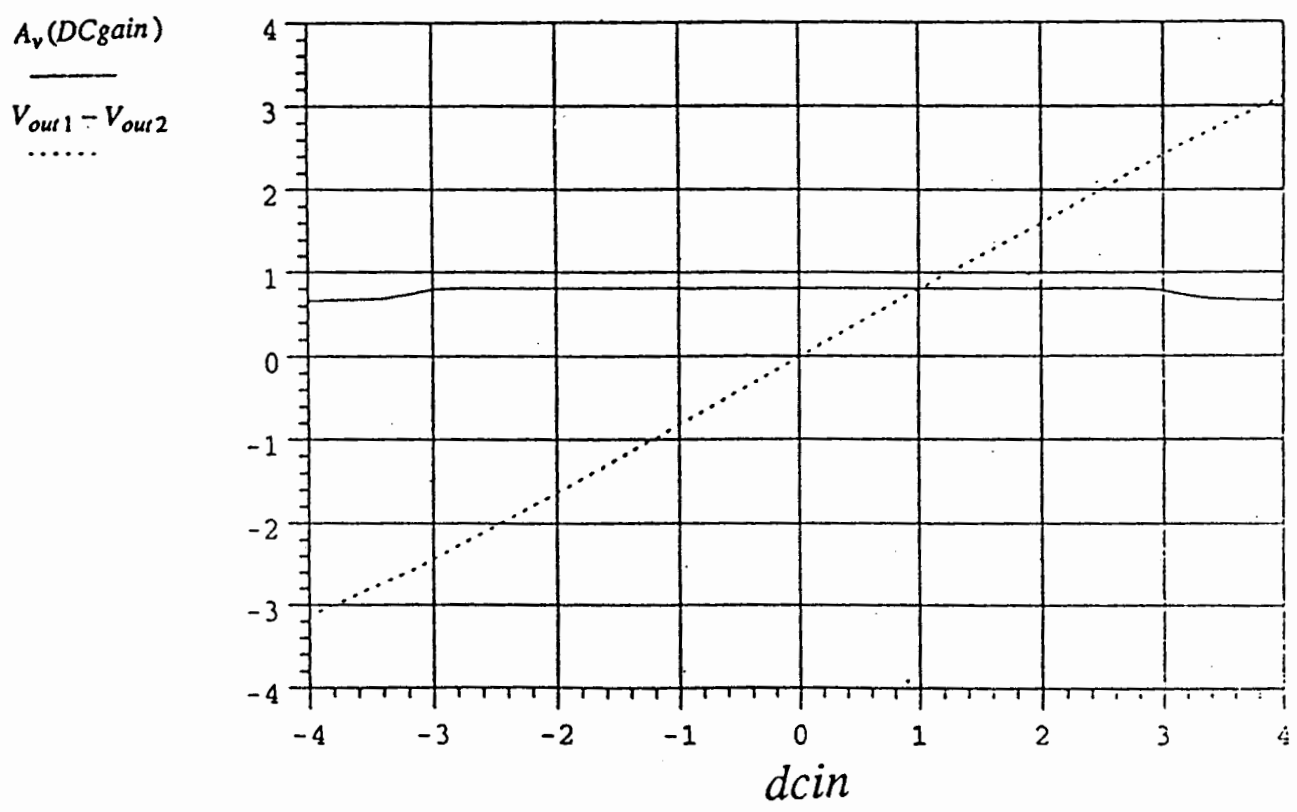

Figure. 18. DC transfer function of the buffer. 
Assuming 3\% device mismatch, the offset is about $4 \mathrm{mV}$. (If we use a single-ended buffer instead of this differential one, the offset will be much larger). Power consumption is about $20 \mathrm{~mW}$. The buffer maintains $0.1 \%$ linearity over a $\pm 2.5 \mathrm{~V}$ input range. The linearity error is defined as

$$
\varepsilon=\frac{V_{\text {out } 1}-V_{\text {out } 2}-A_{v}(0)\left(V_{\text {in } 1}-V_{\text {in } 2)}\right.}{V_{\text {in } 1}-V_{\text {in } 2}} 100 \%
$$

$A C$ performance: For a load of $\mathrm{R}=50 \mathrm{k} \Omega, \mathrm{C}=1 p F, V_{D D}=-V_{S S}=5 \mathrm{~V}$, the buffer's $-3 \mathrm{~dB}$ bandwidth is $260 \mathrm{MHz}$. The single-ended power supply rejection (PSR) for $V_{D D}$ is $67 \mathrm{~dB}$, for $V_{S S}$ is about $55 \mathrm{~dB}$. Common-mode rejection (CMR) is about $55 \mathrm{~dB}$. The cut-off frequency and phase is shown in Fig. 19.

In high-frequency measurements a very small load impedance (usually $50 \Omega$ of the measurement instrument and cable) is often required. In this case, a measurement buffer is necessary. To achieve the required current drive, larger transistors for the output stage are used. For a load of $\mathrm{R}=50 \Omega, \mathrm{C}=10 \mathrm{pF}, f_{-3 d B}=350 \mathrm{MHz}$ which is better than the previous case because of the very small load resistor.

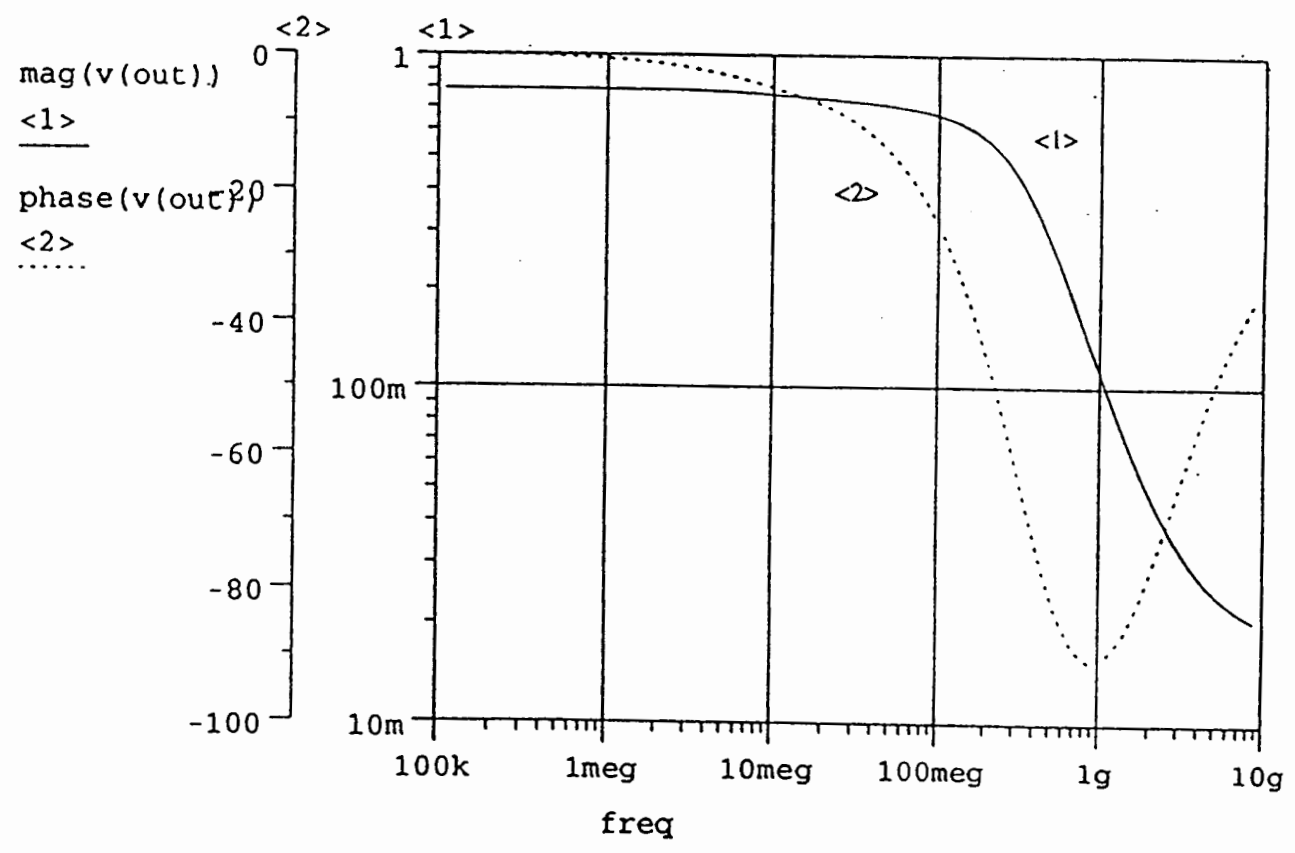

Figure. 19. Frequency response of the buffer. 
Distortion: For a $1 V_{p-p}, f=1 \mathrm{MHz}$ sinewave input signal, the fast Fourier transform yields $0.007 \%$ total harmonic distortion (THD). THD increases to $0.017 \%$ at $f=10 M H z$.

For a lower power supply of $\pm 2.5 \mathrm{~V}$, the buffer bandwidth drops to $130 \mathrm{MHz}$; the linearity error over a $\pm 1.5 \mathrm{~V}$ differential input range was simulated to be $\pm 0.4 \%$. The total harmonic distortion (THD) at $1 V_{p-p}$ and $f=10 \mathrm{MHz}$ was simulated to be $0.02 \%$. Power consumption is as low as $1.4 \mathrm{~mW}$. 


\section{CHAPTER IV}

\section{THE VOLTAGE CONVERTER DESIGN}

In the first three chapters, we designed the basic cells: differential input and output OTA, single-ended input and output transconductance without offset, the "OTA resistor" and an internal (or output) buffer. In this chapter, we will discuss how to design voltage converters. First we consider the single-ended to differential voltage converter.

\subsection{The First Initial Design of a Converter}

To design the converter, the simplest design approach is to make use of the purely differential output OTA designed in Chapter 2 (Fig. 9). The first input of the OTA is tied to the single-ended signal, the second is tired to ground. If we connect two resistors of the same value to the two output nodes of the OTA we should get a purely differential voltage because the two output nodes of the OTA give purely differential output currents. Fig. 20 shows the DC performance of this simple voltage converter. We can see that the linearity is not good. Also the two output voltages are not symmetric.

This simple converter does not work well because the OTA is a symmetric design which also requires a balanced input to cancel nonlinear terms. We can analyze this behavior here. As we know from Eqs. (2.1) and (2.2), the current of a transistor working in saturation is

$$
I_{d}=k\left(V_{g s}-V_{T}\right)^{2}\left(1+\lambda V_{d s}\right)
$$

where

$$
k=\frac{\mu C_{o x} W}{2 L}
$$


For the circuit in Fig. 9, assuming $V_{D D}=-V_{S S}$, the current through $M_{i}$ is $I_{i}$, and labeling $V_{g s 1}=V_{g s 2}=V_{B}$, where $V_{g s 1}$ and $V_{g s 2}$ are the gate-source voltages of transistors $\mathrm{M} 1$ and $\mathrm{M} 2$, and $V_{B}$ is a constant determined by the sizes of $M_{1}-M_{4}$, then

$$
\begin{aligned}
& I_{3}=k\left(V_{i n 1}+V_{D D}-V_{T}\right)^{2}\left(1+\lambda V_{d s 3}\right) \\
& I_{4}=k\left(V_{i n 2}+V_{D D}-V_{B}-V_{T}\right)^{2}\left(1+\lambda V_{d s 4}\right) \\
& I_{5}=k\left(V_{i n 1}+V_{D D}-V_{B}-V_{T}\right)^{2}\left(1+\lambda V_{d s 5}\right) \\
& I_{6}=k\left(V_{i n 2}+V_{D D}-V_{T}\right)^{2}\left(1+\lambda V_{d s 6}\right)
\end{aligned}
$$

and

$$
I_{9}-I_{19}=I_{3}+I_{4}-I_{5}-I_{6}
$$
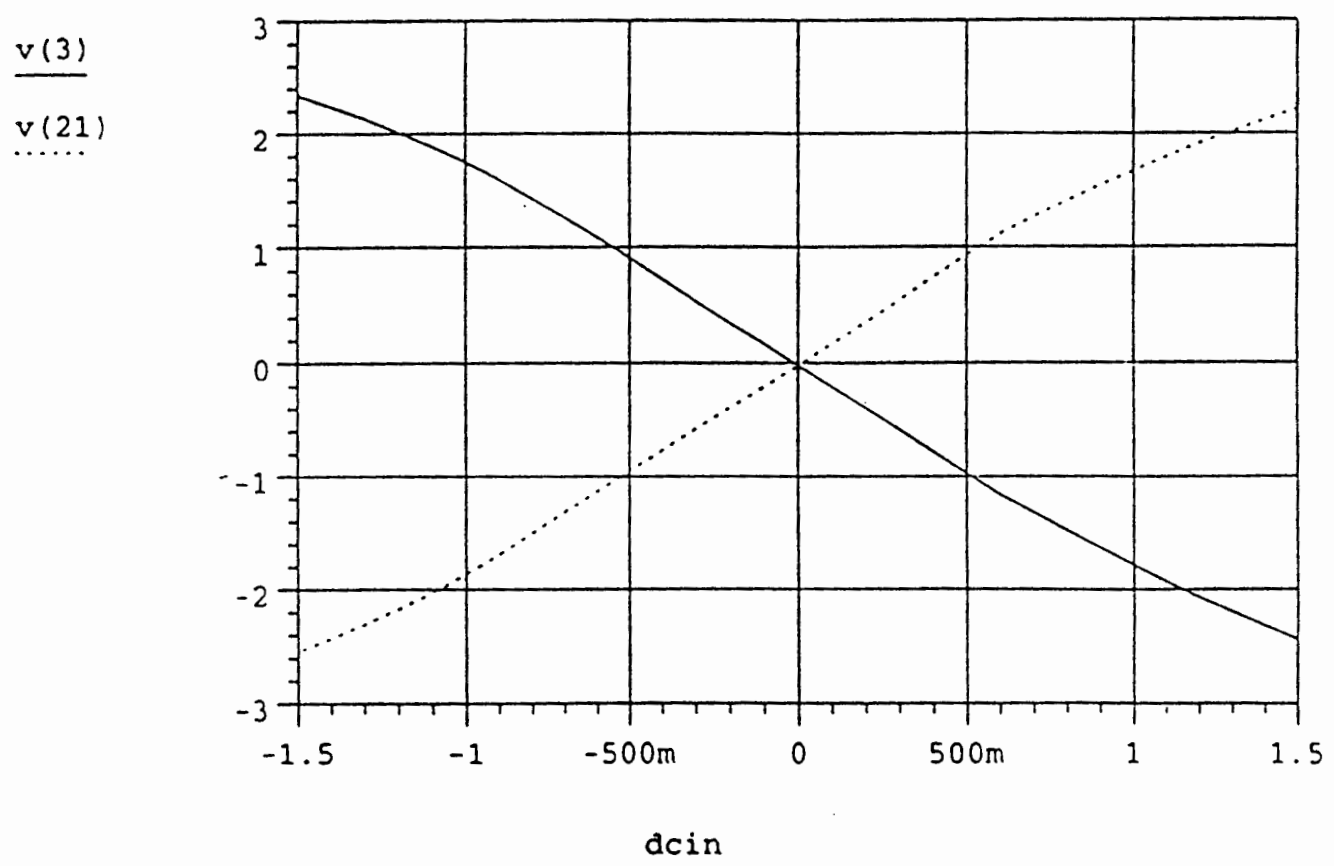

Figure. 20. DC performance of the first initial converter design. $\vec{v}(3), v(21)$ are the two output voltages of converter.

Because $M_{3}$ and $M_{4}\left(M_{5}\right.$ and $\left.N_{6}\right)$ are symmetric in the circuit, we can think that $V_{d s 3}=V_{d s 4}=V_{5}=V_{6}$, then

$$
\begin{aligned}
I_{9}-I_{19}= & k\left(V_{i n 1}+V_{i n 2}+2 V_{D D}-2 V_{T}\right)\left(1+\lambda V_{d s 3}\right)\left(V_{i n 1}-V_{i n 2}\right) \\
& +k\left(V_{i n 1}+V_{i n 2}+2 V_{D D}-2 V_{B}-2 V_{T}\right)\left(1+\lambda V_{d s 4}\right)\left(V_{i n 1}-V_{i n 2}\right) \\
= & k\left(V_{i n 1}-V_{i n 2}\right)\left(1+\lambda V_{d s 4}\right)\left(2 V_{i n 1}+2 V_{i n 2}+4 V_{D D}-2 V_{B}-4 V_{T}\right)
\end{aligned}
$$




$$
=k\left(1+\lambda V_{d s 4}\right)\left(V_{\text {in } 1}-V_{\text {in } 2}\right) F_{t}
$$

where

$$
F_{l}=\left(2 V_{i n 1}+2 V_{i n 2}+4 V_{D D}-4 V_{T}\right)
$$

If $F_{t}$ is constant (independent of $V_{i n 1}, V_{i n 2}$ ), the OTA has good linearity. The only way $F_{t}$ can be constant is $V_{\text {in } 1}=-V_{\text {in } 2}$. That means the input would be purely differential,

$$
I_{9}-I_{19}=4 k\left(V_{\text {in } 1}-V_{i n 2}\right)\left(1+\lambda V_{d s 4}\right)\left(V_{D D}-V_{T}\right)
$$

to get the best linearity. We can estimate from (4.6) that when $V_{i n 2}=0$,

$$
I_{9}-I_{19}=F_{1} V_{\text {in } 1}+F_{2} V_{i n 1}^{2}
$$

where

$$
\begin{aligned}
& F_{1}=k\left(1+\lambda V_{d s 4}\right)\left(4 V_{D D}-4 V_{T}\right) \\
& F_{2}=2 k\left(1+\lambda V_{d s 4}\right)
\end{aligned}
$$

Assuming $V_{\text {in } 1}=0.5 \mathrm{~V}$, from (4.9) the additional nonlinearity error is about $5 \%$. This is why for a purely differential input, the circuit has much better performance than for a single-ended input. Even if it is not a purely differential input, e.g., $V_{\text {in } 2}=-0.9 V_{\text {in } 1}$, from (4.9) and (4.10) the linearity of the OTA is still ten times better than the single-endedinput OTA.

\subsection{The Second Initial Design of a Converter}

We already designed a good buffer, we also have a single-ended input and output OTA. Now we use these basic cells to design a simple voltage converter. We know the transconductance cell, $G_{m}$, has a transconductance $g_{m}$, and if we connect the input and output of this $G_{m}$ together, we get a resistor $R_{l}$ of a value of $1 / g_{m}$. If we use two identical transconductance cells, as shown in Fig. 21 , with an input voltage $V_{i n}$, the first cell gives a current $I_{o}=-g_{m} V_{i n}$, the current flows through the second cell to produces an output voltage 


$$
V_{o}=I_{o} R_{l}=\left(-g_{m} V_{\text {in }}\right)\left(1 / g_{m}\right)=-V_{\text {in }}
$$

So we can use two transconductance cells to build a voltage inverter. The cells used are the improved $G_{m}$ cells of Fig. 13 where the DC offset has been deleted (see Section 2.3).

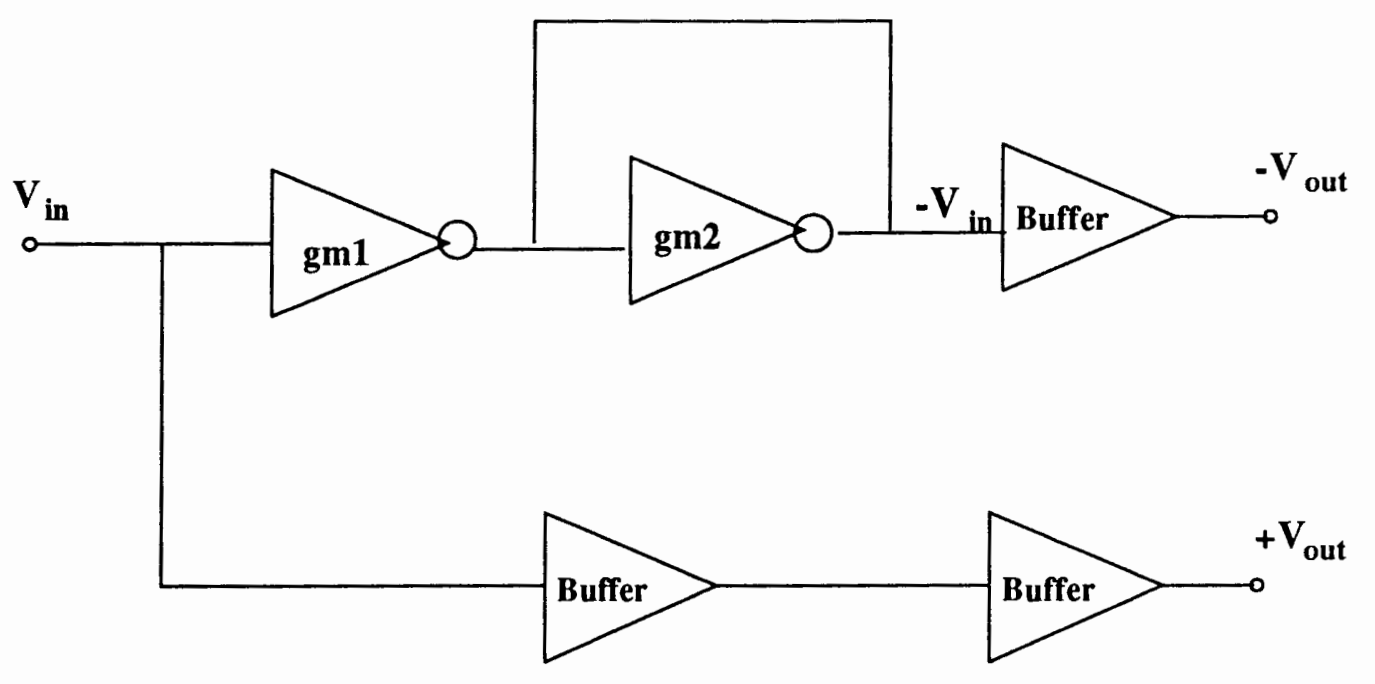

Figure. 21. The principle circuit of the second converter design.

We used SPICE to simulate this voltage converter and found the inverter had good linearity and very wide bandwidth. But the output voltage was smaller than $V_{\text {in }}$ (about $0.9 V_{i n}$ ). This is because the $G_{m}$ not ideal, i.e., it also has an output impedance $1 / g_{o}$. The load can be expressed approximately as $1 /\left(g_{m 2}+g_{o 1}+g_{o 2}\right)$ and the inverter output voltage is

$$
V_{o}=\frac{-g_{m 1}}{g_{m 2}+g_{o 1}+g_{o 2}} V_{i}, \quad\left(g_{m 1}>g_{m 2}\right)
$$

To get an accurate voltage inverter, we can choose larger size transistors for the first cell, to provide higher current gain to the second cell. We use the voltage inverter to invert $V_{\text {in }}$, also we use a buffer to get a delayed $V_{\text {in }}$, so the positive and negative outputs basically have the same amplitude and delay. Both outputs connect with an output buffer. By this way, we get a purely differential voltage. 
The advantages of this design are:

A. The circuit is very simple (no current mirrors), both the OTA and the buffer are simple. The working frequency is very high. With a load of $50 \mathrm{k} \Omega$ and $0.1 p F, f_{-3 d B}$ is simulated to be above $300 \mathrm{MHz}$.

B. If the next stage does not have a large load, the converter does not need an output buffer. The design is further simplified and can work at higher frequences ( > $500 \mathrm{MHz})$.

The shortcoming is that the differential signals come through two different paths; it is difficult to keep the two signals at exactly the same amplitude and phase.

\subsection{Improved Cascade Converter Design}

As we discussed above, the two initial designs of voltage converters fall short in obtaining purely differential voltages. The first design did not use its intrinsic balanced circuit operation, the signal was not purely differential but it did have the ability to improve the imbalance internally and give a differential-like output voltage (see the simulation for this circuit in Fig. 19). The second design fails in a giving purely differential voltage because it processes signals through different paths for the two outputs, but it has a simple circuitry and very high frequency response. Understanding these reasons provides us a solution: a combination of these two designs should result in a good circuit.

We build a cascade-structure converter, see Fig. 21. The first stage is the simple converter we discussed (see Fig. 20) and provides a differential-like input voltage for the second stage. The second stage is the initial Design 1 of the converter we discussed in Section 4.1. But the second stage converter does have a differential-like input. We expect that the second stage improves the input signal greatly in both $D C$ and $A C$ performance.

We used SPICE to simulate this cascade-structure converter. The device process 
parameters are the same as used before (see Section 3.3), with a $10 k \Omega$ resistor and a $100 f F$ capacitor as load. Fig. 22 shows the DC transfer function of the converter. Compared to Fig. 19 (single-end input) we can see that the new design gives better linearity and lower offset. And the two outputs are purely differential voltages in a range of $\pm 0.5 \mathrm{~V}$.

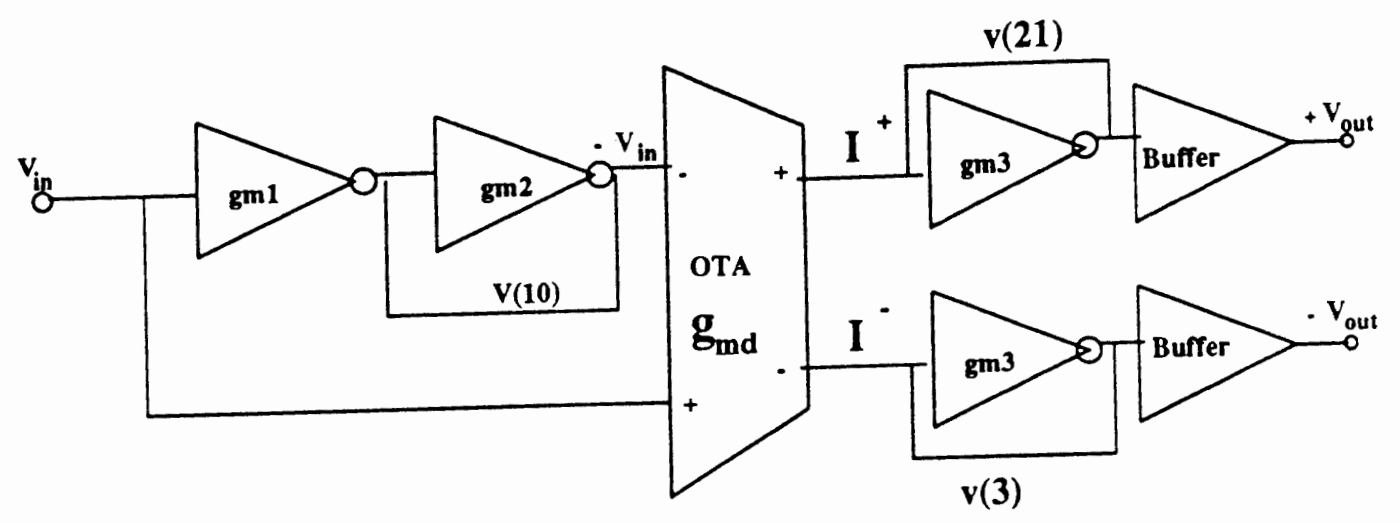

Figure. 22. The principle circuit of the cascade voltage converter.

Fig. 23 gives the $\mathrm{AC}$ performance for this converter. We can see that now the two outputs have almost the same magnitude and bandwidth. The cut-off frequency is above $100 \mathrm{MHz}$. For a $1 V_{p-p}, f=10 \mathrm{MHz}$ sinewave input signal, the fast Fourier transform yields $0.5 \%$ total harmonic distortion. The voltage and phase values at differential frequencies are given in Table I and Table II. From the tables, we can have a closer look at the improved design. We compare the voltage and phase at the input of first stage, the inputs of the second stage $\left(\left(V_{i n}, \mathrm{~V}(10)\right)\right.$, and the outputs after the second stage $(\mathrm{V}(3), \mathrm{V}$ (21)). In the table, the phase error is the phase shift compared to the initial input signal. If two voltages are purely differential, they should have the same magnitude and same phase error, and have a constant phase difference: $180^{\circ}$.

From the tables we can see that the initial input is single-ended input. After the first stage results in differential-like voltages which are far from purely differential in 


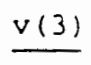

$v(21)$

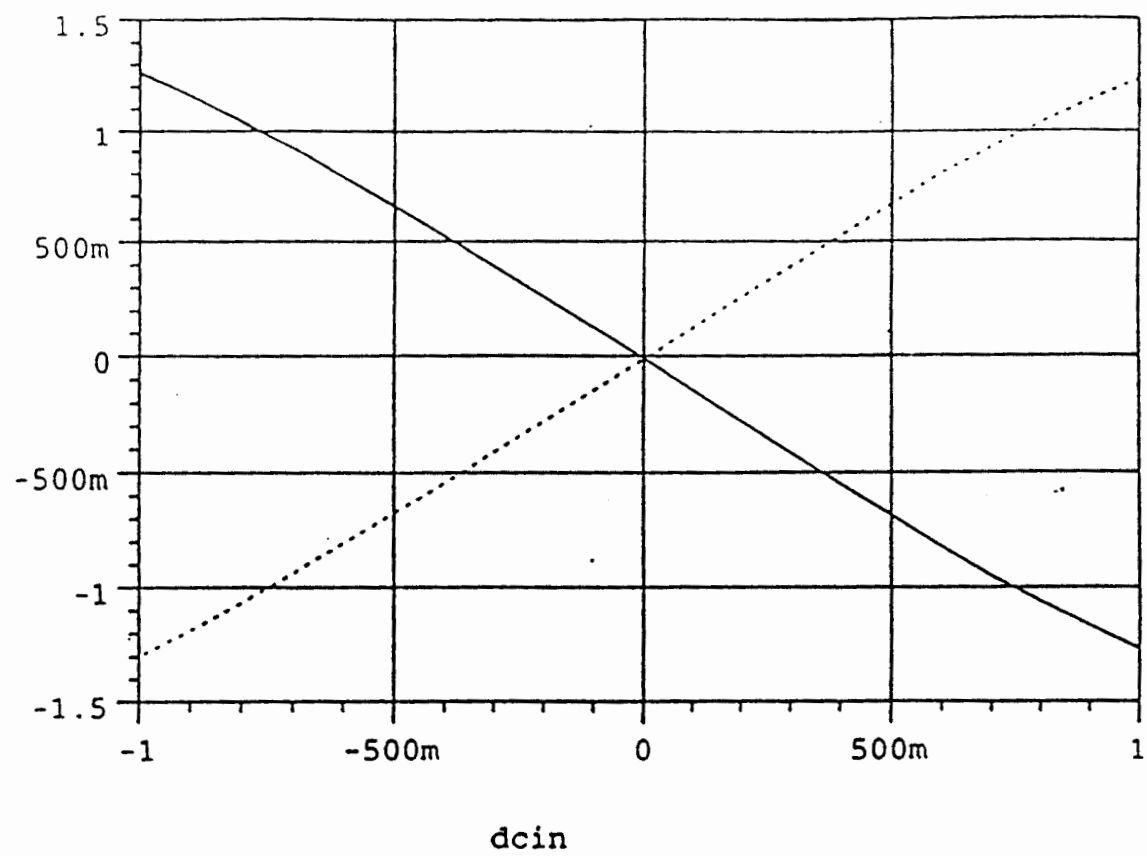

Figure. 23. DC performance of the cascade voltage converter. $\mathrm{v}(3), \mathrm{v}(21)$ are the two output voltages of converter.

v3

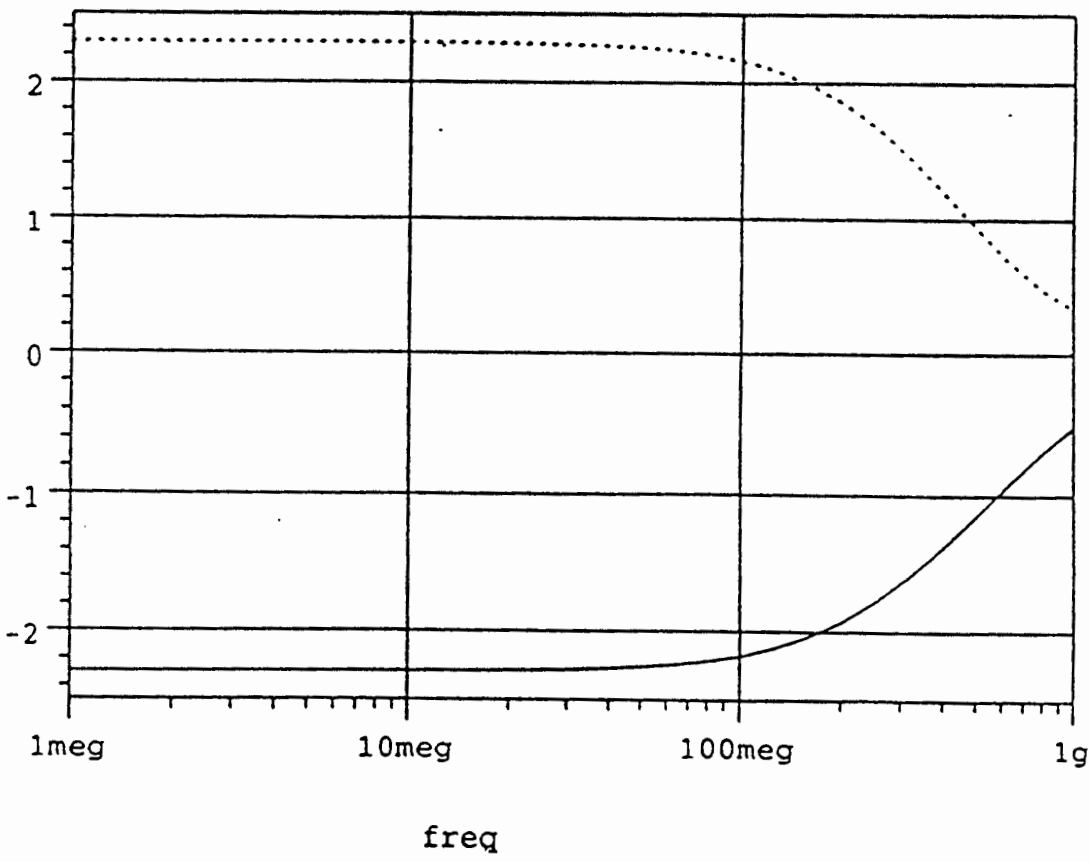

Figure. 24. AC performance of the cascade voltage converter. $\sqrt{3}, \mathrm{v} 21$ are the magnitudes of the two output voltages of converter. 
TABLE I

CONVERTER INPUT/OUTPUT VOLTAGES AT DIFFERENT FREQUENCIES

\begin{tabular}{|lrrll|}
\hline \multicolumn{5}{c|}{$\begin{array}{c}\text { Voltage at Inputs and Outputs } \\
\text { (units:volts) }\end{array}$} \\
\hline Freq(MHz) & Mag(Vin) & Mag(V(10)) & Mag(V(3)) & Mag(V(21)) \\
\hline 1 & 1 & 0.973 & 2.295 & 2.295 \\
5 & 1 & 0.972 & 2.295 & 2.295 \\
10 & 1 & 0.972 & 2.291 & 2.292 \\
16 & 1 & 0.971 & 2.288 & 2.288 \\
20 & 1 & 0.971 & 2.285 & 2.286 \\
25 & 1 & 0.971 & 2.281 & 2.281 \\
32 & 1 & 0.971 & 2.276 & 2.278 \\
40 & 1 & 0.971 & 2.267 & 2.271 \\
50 & 1 & 0.970 & 2.255 & 2.260 \\
63 & 1 & 0.970 & 2.235 & 2.244 \\
80 & 1 & 0.969 & 2.206 & 2.219 \\
100 & 1 & 0.968 & 2.160 & 2.181 \\
125 & 1 & 0.966 & 2.093 & 2.124 \\
200 & 1 & 0.959 & 1.858 & 1.929 \\
316 & 1 & 0.942 & 1.455 & 1.593 \\
\hline
\end{tabular}

TABLE II

CONVERTER INPUT/OUTPUT PHASES AT DIFFERENT FREQUENCIES

\begin{tabular}{|lllll|}
\hline \multicolumn{5}{c|}{$\begin{array}{c}\text { Phases Error of Inputs and Outputs } \\
\text { (units: degrees) }\end{array}$} \\
\hline Freq(MHz) & Ph(Vin) & Ph(V(10)) & Ph(V(3)) & Ph(V(21)) \\
\hline 1 & 0.0 & 0.07 & 0.31 & 0.31 \\
5 & 0.0 & 0.33 & 1.58 & 1.57 \\
10 & 0.0 & 0.62 & 3.13 & 3.11 \\
16 & 0.0 & 0.94 & 4.91 & 4.89 \\
20 & 0.0 & 1.17 & 6.16 & 6.13 \\
25 & 0.0 & 1.45 & 7.73 & 7.69 \\
32 & 0.0 & 1.82 & 9.69 & 9.64 \\
40 & 0.0 & 2.27 & 12.16 & 12.10 \\
50 & 0.0 & 2.85 & 15.26 & 15.18 \\
63 & 0.0 & 3.59 & 19.13 & 19.03 \\
80 & 0.0 & 4.51 & 23.97 & 23.83 \\
100 & 0.0 & 5.67 & 29.97 & 29.79 \\
125 & 0.0 & 7.12 & 37.37 & 37.10 \\
200 & 0.0 & 11.23 & 57.19 & 56.52 \\
316 & 0.0 & 17.59 & 84.78 & 82.73 \\
\hline
\end{tabular}


both magnitude and phase. After the second stage, the voltages are very close to purely differential. The magnitude and phase errors between the two outputs have a low asymmetry $(<1 \%)$ until very high frequencies $(>50 \mathrm{MHz})$. Both signals have a bandwidth of about $200 \mathrm{MHz}$. The simulation shows that the cascade design is an effective way to obtain a high-frequency, purely differential voltage. If we consider other parasitic capacitors and resistors, the bandwidth would get worse; we will consider this issue in the next chapter. 


\section{CHAPTER V}

\section{LAYOUT OF THE CONVERTER CHIP}

\subsection{MOSIS Process and the Layout Tool}

After finishing the schematic design and simulation, we created the layout for the converter chip and sent the design out for fabrication. Since this is an analog circuit design, there is no software tool available that automatically converts the circuit schematics to the layout. On the other hand, since this is a very-high-frequency circuit, a very careful layout design must be performed to assure the circuit's good performance. This is a good chance for learning how to design the layout for high-frequency circuits.

We use MOSIS's process to manufacture the chip. This is $2 \mu \mathrm{m}$ CMOS process, i.e., the minimum gate length of transistors is $2 \mu \mathrm{m}$. The parameters of this process are given in Appendix I. It is a double-metal, double-poly, n-well process and the chip package has 40 pins.

We used the "Magic" tool to create the layout. Magic has plenty of commands to zoom, copy, rotate, label, etc., for the layout, which make the layout process very convenient. It can also automatically check the layout to see if the design meets the design rules, and give warnings if there are problems.

Magic uses different colors to represent different layers and materials, so the whole layout looks clean and is easy to check. It can automatically calculate the parasitic capacitance and resistance at every node and connection. If the design is a digital circuit, digital simulation can be done in Magic. If the design is an analog circuit, Magic can not do analog simulation directly, but can use circuit extractor in Magic to get the complete 
netlist of the circuit (including the parasitic capacitance at every node). Then based on this netlist the analog simulation can be performed with analog simulators such as SPICE, TSPICE or SABER. The simulation can be very close to the real case because of the good calculation of parasitic capacitance.

The layout of power lines must be taken into consideration during the layout stage. There are many power lines in the layout. To conserve silicon area, we tried to use narrower power lines, but they must be wide enough to carry the maximum working current the circuit needs, otherwise the power lines may be burned (or the chip may be burned), or there is significant voltage drop on the power lines [17] and the circuit can not work well. For this design, the maximum current is less than $50 \mathrm{~mA}$ and there are three power lines, the minimum width of the power line should be chosen as $8 \mu \mathrm{m}$.

\subsection{Latch-up Prevention}

Without special attention in the layout design of the CMOS process, a parasitic circuit effect called "latch-up" may happen. Latch-up is a common and important phenomenon in CMOS structures. It can not be observed from circuit design and, therefore, can only be avoided in layout design.

Fig. 25a shows a cross-section of a P-well CMOS structure, building on a $\mathrm{N}$ substrate and Fig. 25b shows its top view [17]. On the left side of the figure, there is a PMOSFET containing the P-type drain/source diffusions, poly gate, and the aluminum interconnection. In the P-well, there is an NMOSFET which has a similar structure as the PMOSFET, except that N-type, instead of P-type, diffusions are used for the drain/source. In order to isolate these two devices, a thick silicon oxidation is used for a high threshold voltage.

Beside the above-mentioned real devices, there exist parasitic elements. One parasitic PNP lateral bipolar transistor is formed by P-diffusion, N-substrate, and P-well. 


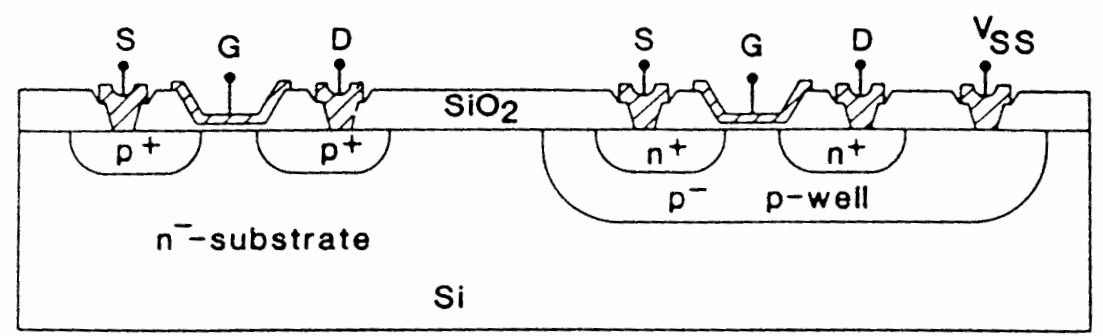

(a)

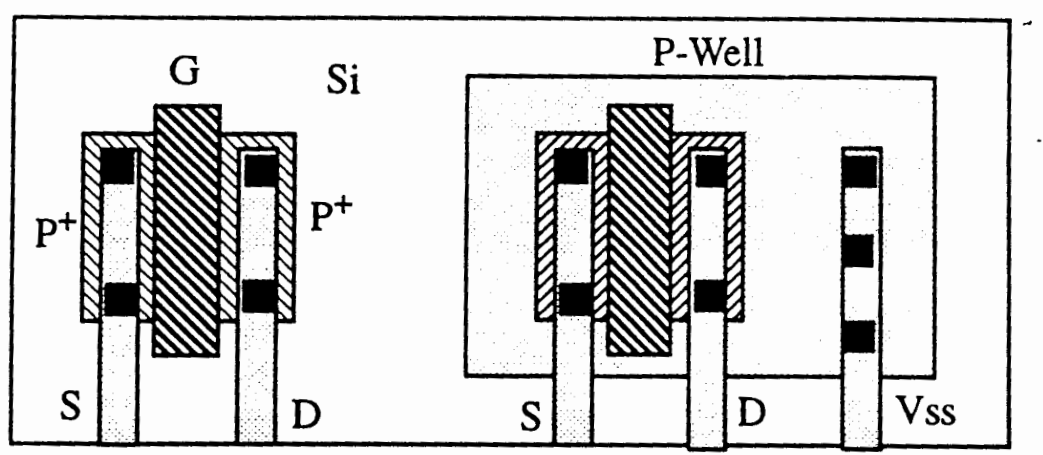

(b)

Figure. 25. A cross-section of the layout of a $p$-well CMOS structure (a) and its top view (b).

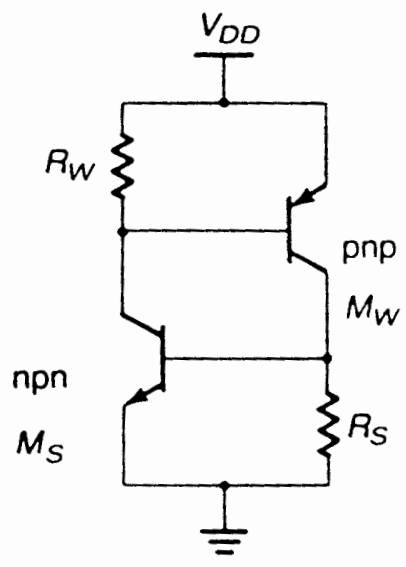

Figure. 26. Basic latch loop formed by parasitic bipolar transistors and parasitic resistors in CMOS structure. 
Another parasitic NPN vertical transistor is built by N-substrate, P-well, and N-diffusion. These two transistors form a pnpn structure and are connected to each other as shown in Fig. 26 with $R_{W}$ as the well resistance and $R_{S}$ as the substrate resistance. From Fig. 26, it can be derived that, if both of the base-emitter junctions of the bipolar devices are forward biased and if the loop gain satisfies the condition

$$
\beta_{N P N} \beta_{P N P} \geq 1
$$

with $\beta_{P N P}$ and $\beta_{N P N}$ being the current gains of the two transistors, the currents in both transistors will increase until they reach self-limit or until they result in the destruction of the chip or its bonding leads. This phenomenon is called CMOS latch-up [17 - 19]. The result of this effect is shorting of the $V_{D D}$ and $V_{S S}$ lines, resulting in chip self-destruction or at least system failure with the requirement to power down. We must take steps to prevent the effect from happening.

The way to reduce the latch-up risk is to to reduce the resistances $R_{W}$ and $R_{S}$ so that the forward base-emitter biases of the NPN transistor and of the PNP transistor are less than $0.6 \mathrm{~V}$ and, therefore, these transistors are off. The method to reduce these resistances is to place a P+ collar (called a guard ring) around the periphery of the P-well or an $\mathrm{N}+$ guard ring surrounding the P-channel device. It costs circuit density.

In our design we took several steps to reduce latch-up risk:

* Every n-well had substrate contacts with $V_{S S}$. We placed one substrate contact per 10-15 transistors.

* Every substrate contact should be connected by metal directly to a supply pad (i.e., no diffusion or poly).

*Substrate contacts were placed as close as possible to the source connection of transistors connected to the supply rail (i.e., $V_{S S}$ n-device, $V_{D D}$ p-device).

The most likely place for latch-up to occur is in the I/O structures where large currents 


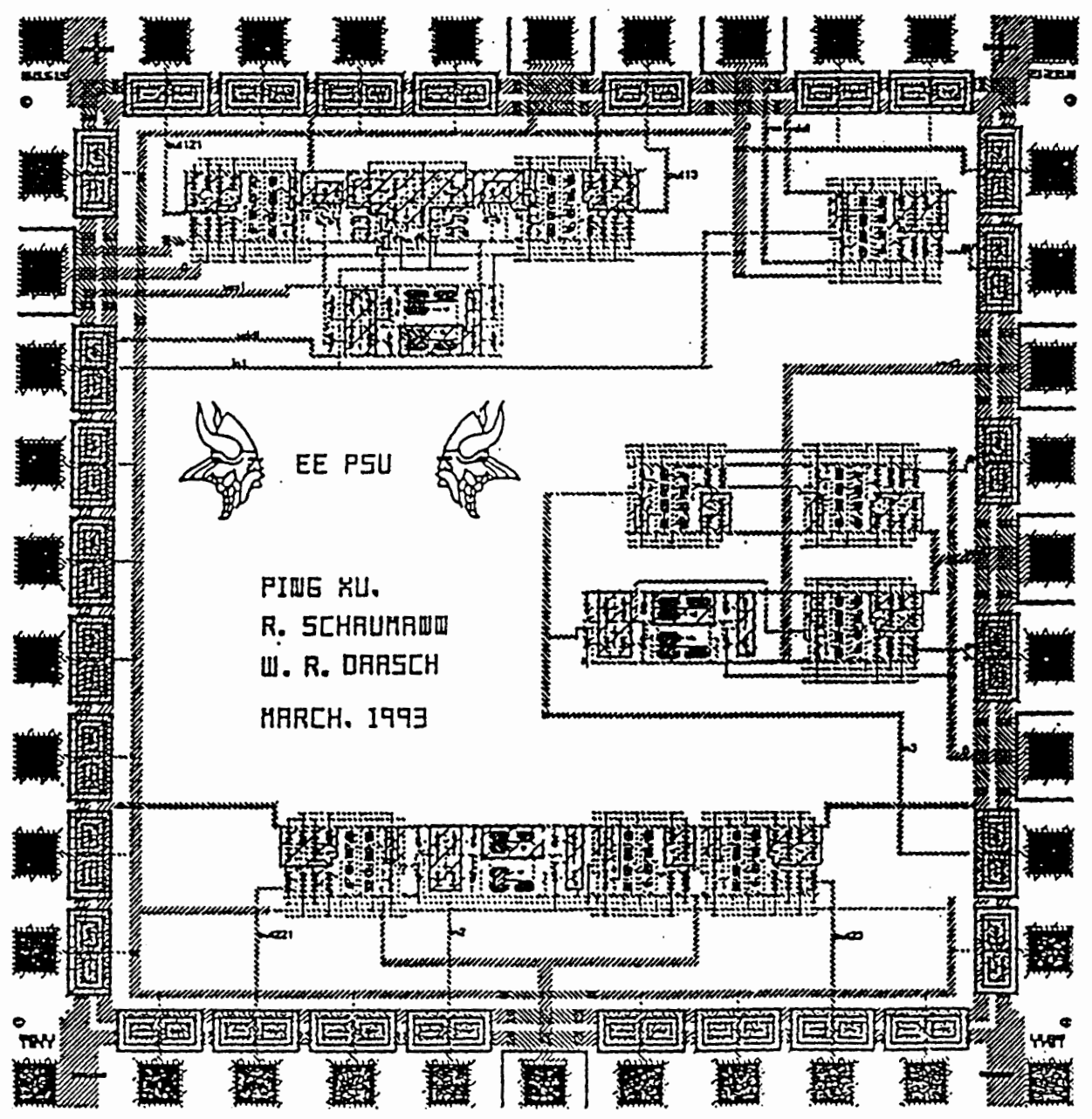

Figure. 27. The Magic layout of the converter chip. 
flow, large parasitic effects may be present and abnormal circuit voltages may be encountered. In our design, the output of OTAs and out buffers needed more attention. Guard rings are applied to the layout design of the converter. $\left(n+\right.$ guard rings connected to $V_{D D}$ around p-transistors). This effectively reduces the parasitic resistance, so the risk of latch-up is reduced to minimum. Fig. 27 shows the layout of the chip.

\subsection{Reducing the Parasitic Capacitance.}

Since we designed a very-high-frequency circuit, it is very sensitive to the load or parasitic capacitance. It can be seen from Fig. 26 that there is a parasitic capacitance between diffusion and substrate/well associated with the drain and source terminals of a device. In practice, this kind of capacitance cannot be avoided and the value of the capacitance depends on doping, applied voltage, and diffusion area. The first and second parameters cannot be changed in the layout design, but the third parameter can. We tried to reduce the diffusion area.

Another kind of parasitic capacitance is wire capacitance which exists between poly/metal to substrate, poly2 to poly1, metal to poly, and metal2 to metal1. This kind of capacitance depends only on the area, so that the only way to reduce the capacitance is to use a narrow and short interconnection. We should use the smallest contact available between two layers. For some critical nodes, we try to use shortest and thinnest connecting wires.

The basic cells of the circuit are OTAs, which usually do not have a buffered output stage. From simulation of some OTAs, we learned that sometimes even a $50 \mathrm{fF}$ additional capacitance at some critical nodes will reduce the bandwidth by more than a factor of 2 . To solve this problem sometimes we can apply a special buffer such as we discussed in Chapter 3 to help improve the driving capability. More often, we need to pay extremely close attention to layout design to reduce the parasitic capacitance. If a 
capacitance exists between two signal lines, it will cause crosstalk, which means the signal in one line is being passed to another line in error [20 - 22]. To reduce crosstalk, routing two lines in parallel should be avoided. Also, the space between two wires may need to be large for reducing the parasitic capacitance.

In the beginning, I did not pay attention to these details in layout. The final result showed the cut-off frequency dropped about $75 \%$ compared to the pre-layout simulation. The layout had to be redesigned.

\subsection{Extracting and Simulating the Whole Chip Layout}

After we finished the layout of the whole chip (see Fig. 27), we wanted to do the simulation based on the whole chip. We extracted the whole chip. It gave a detailed netlist (SPICE file) of the whole chip, including the parasitic capacitance at every node. After modifying the netlist (getting rid of some meaningless symbols), the SPICE file can be used to do analog simulation. Because I am more familiar with TSPICE (Tektronics SPICE), I used a function in TSPICE to transfer the SPICE file into TSPICE file, and did the simulation in TSPICE.

The layout brought in large parasitic capacitances at every node. This did affect the circuits AC performance, but not have effect on DC performance (see Fig. 28). Fig. 29 gives the AC performance of the chip. Comparing that with the performance before the layout (see Fig. 24), we can see how much change occurred. As we know, the parasitic capacitance caused this poor AC performance. We need to make every effort to reduce the parasitic capacitance.

When we simulated the circuit with a load of $R=50 \Omega, C=10 p F$, which are the values used in typical high-frequency measurements, the design gave a bandwidth of $130 \mathrm{MHz}$, and we observed purely differential output. 
v 3

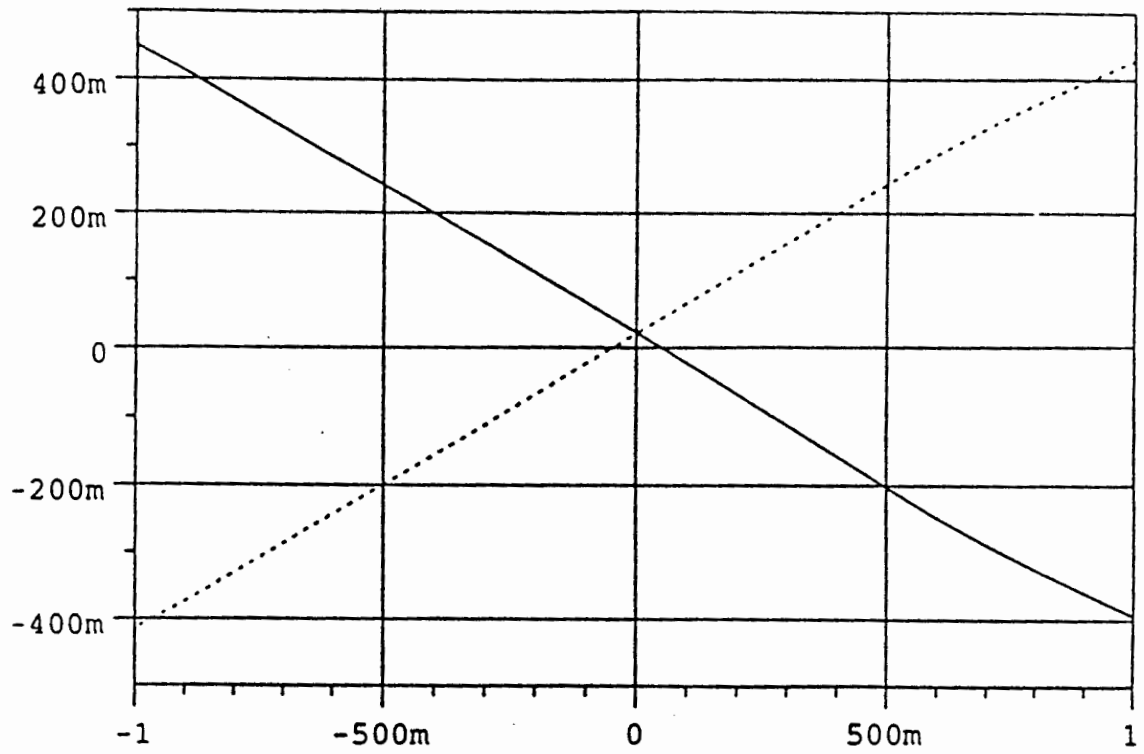

dein

Figure. 28. SPICE simulation (DC) of the converter chip layout $\sqrt{3}, \mathrm{v} 21$ are the magnitudes of the two output voltages of converter.
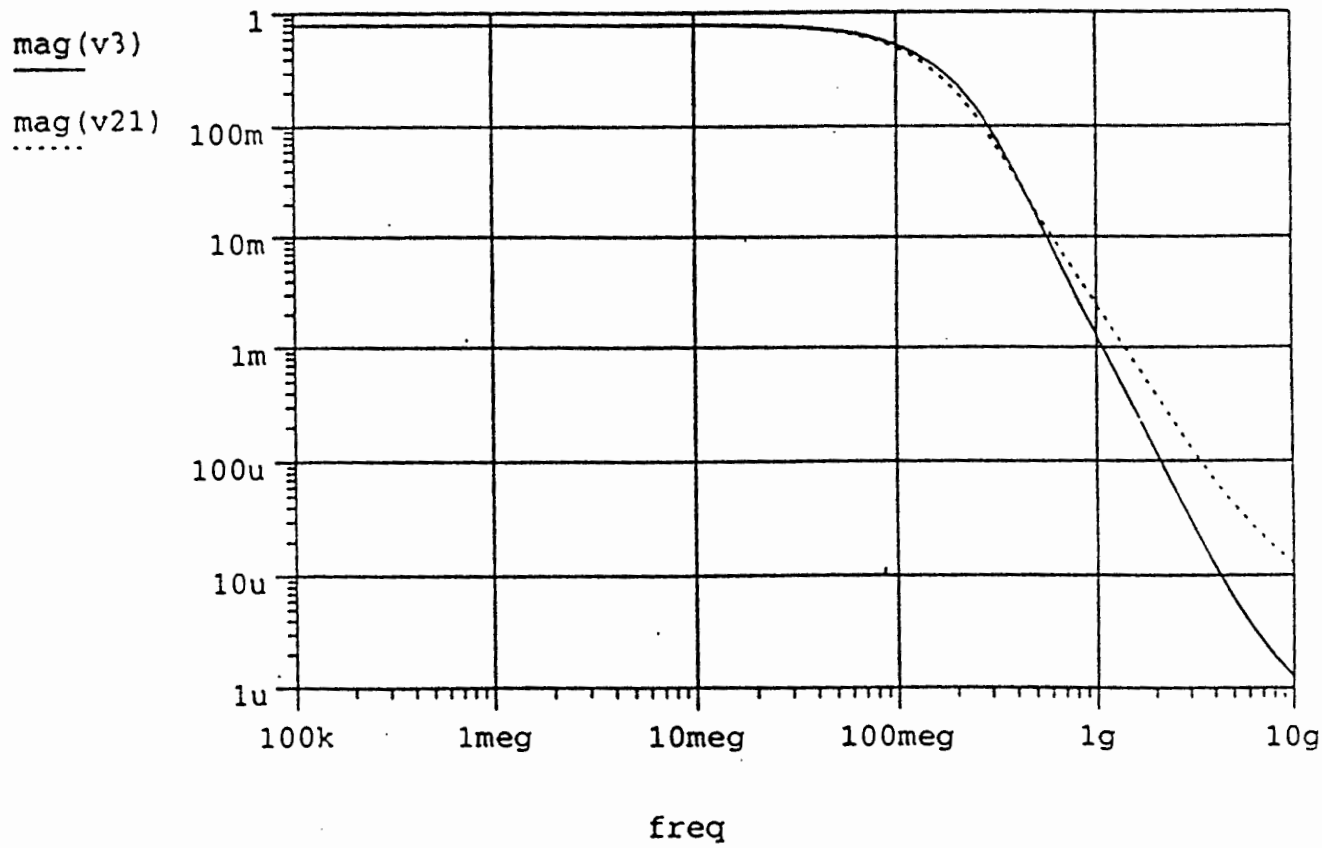

Figure. 29. SPICE simulation (AC) of the converter chip layout. mag(v3), mag(v21) are the magnitudes of the two output voltages of converter. 


\section{CHAPTER VI}

\section{THE MEASUREMENT OF THE CHIP}

\subsection{The Difficulty of High-Frequency Measurements}

after we finished the layout design of the chip the design (layout) was sent to MOSIS (at the University of Southern California) to be fabricated. MOSIS (MOS Implementation Service) is a group of vendors operated by the Information Sciences Institute of the University of Southern Califomia under the sponsorship of the U.S. Department of Defense Advanced Research Project Agency (DARPA) and the National Science Foundation (NSF). The feature of MOSIS is that it combines several projects on a single wafer fabrication run, so the cost can be reduced. Details of the MOSIS process file we used for SPICE simulations are shown in the Appendix I.

We received four chips for measurement. We complete the measurements in the Circuits Lab in the Department of Electrical Engineering at Portland State University, and in the Intel Network Circuits Lab. There are many reasons that cause chips to fail such as design problems, manufacturing process problems, or measurement mistakes. It is hard to get the designed chip to work the first time.

Many design and simulation tools can help reduce the risk, but still some failure is out of the engineer's control. We must do a careful job at every stage to assure the chip is working even under the worst case conditions, or at least working partly.

Because the circuit layout was small, I put three similar circuits on the chip. Each circuit has separate inputs, outputs and power supplies. In case part of the circuit had problems, the other circuit still may work. 
A very-high-frequency circuit measurement is extremely difficult. Not only is there a limitation of measurement instruments, but also we need to consider such factors as large parasitic capacitance brought in by connection wires, test board and probes, noise brought in by power lines, and the impedance mismatch between the measured circuit and measurement instruments.

\subsection{The Measurement Process}

Since this circuit itself is a voltage converter, it does not need high-frequency transformers to provide the differential voltage and the design does not need other power supplies or bias voltages except $V_{D D}$ and $V_{S S}$ (and DC Ground), the measurement setup is very simple. We chose a gold-dressed chip socket for this chip. This was expensive but it assured that the socket has a very good contact with the pins of the chip.

The expanded photograph of the chip die is shown in Fig. 30). Clean (low noise) power and ground lines were very important for the measurements (see Fig. 31). For this chip, all unused pins were connected to ground. The power lines were connected with $0.1 \mu F$ by-pass capacitors to filter the noise and keep the power lines stable. The by-pass capacitors were connected as close as possible to the input pins of the chip and the capacitor leads were as short as possible (long lines or leads introduce parasitic inductance). For the same principle, the "ground" was connected well to system ground with thick connecting wires.

Another consideration is that we need to match input and output impedance at the measuring node when we do the measurement. For the coaxial cables and BNC connector, the impedance is $50 \Omega$, so we put a $50 \Omega$ resistor at input and output of the chip to match the cable impedance.

The first test board was not designed well. The power and ground lines were not very clean, and BNC connectors were not used for the input and output nodes, the input 


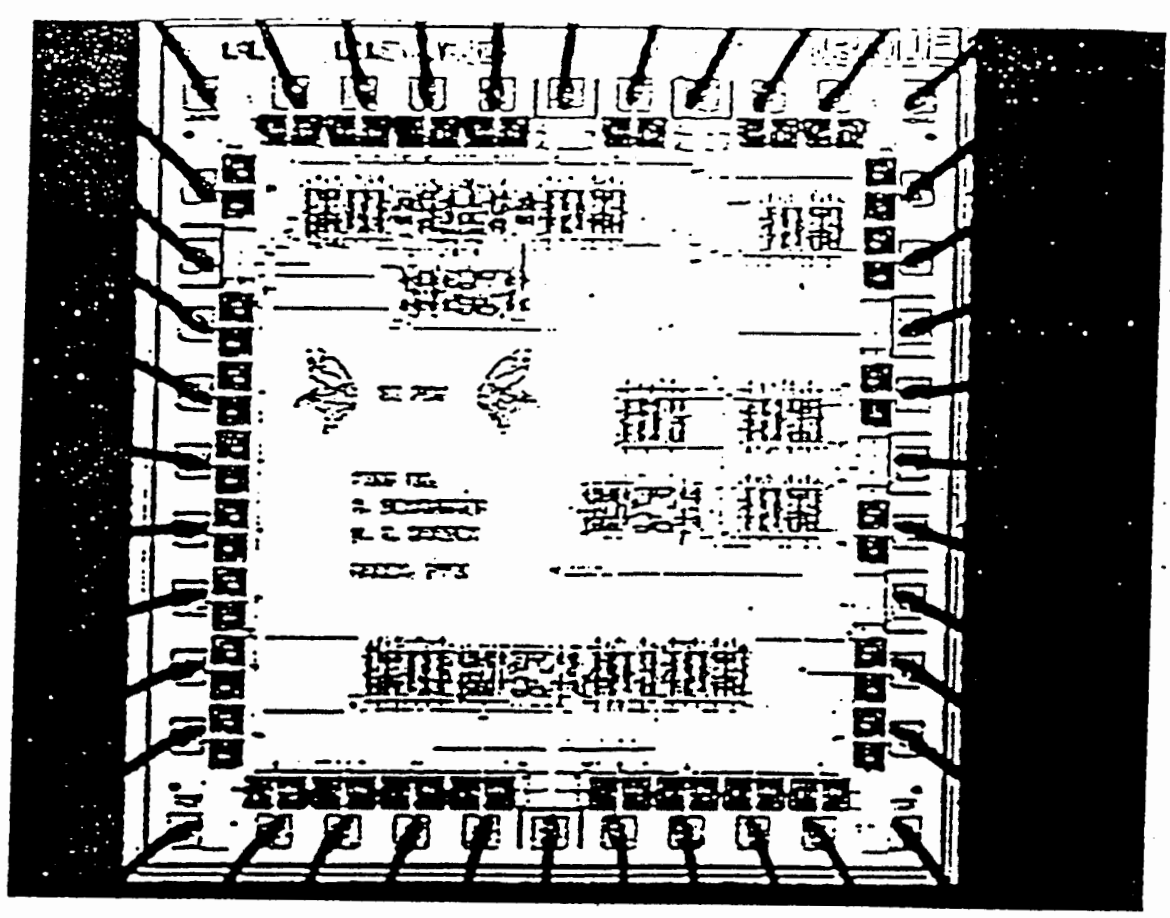

Figure. 30. The photograph of the chip die.

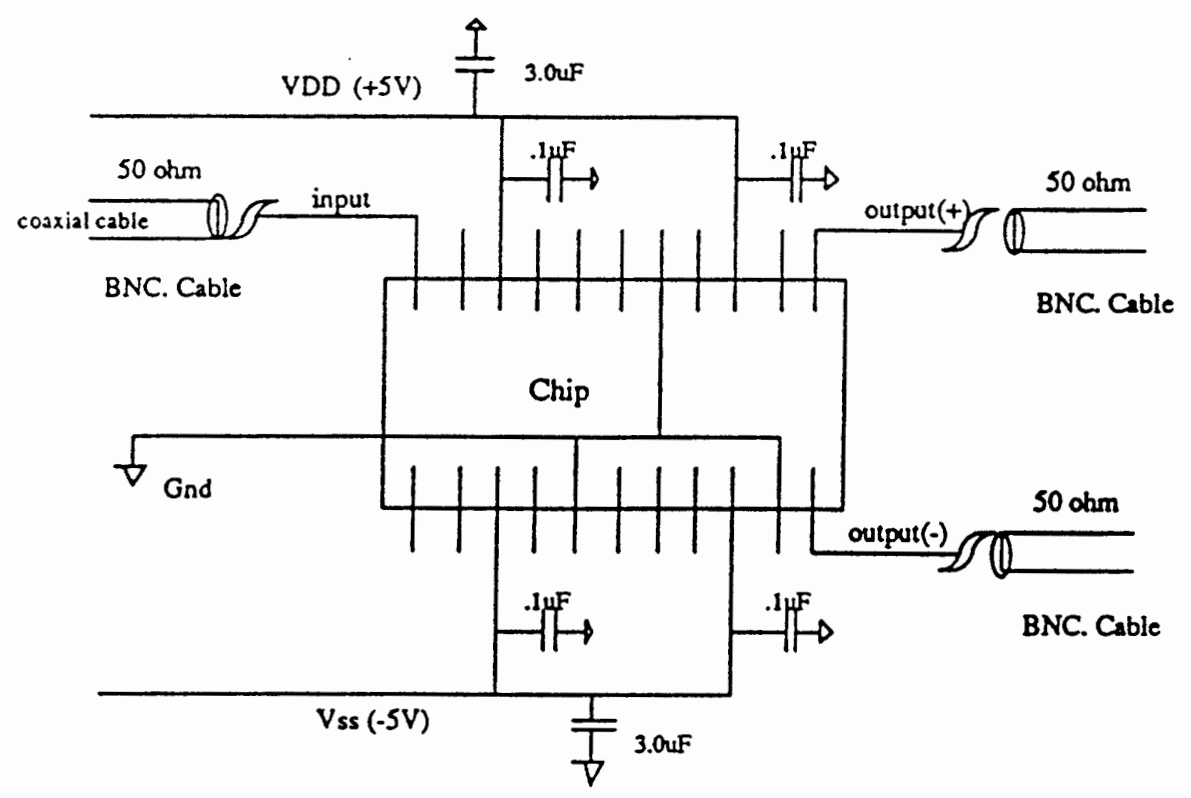

Figure. 31. The measurement set-up for the converter chip. 
and outputs were so corrupted by noise, no bandwidth measurements were possible.

A second test board was built. Learning lessons from the first one, more attention is paid to get much cleaner power and ground lines, BNC connectors and coaxial cables were used at input and output nodes, which prevented the signal from leaking and from coupling outside noise sources. For a much higher frequency measurement $(52 \mathrm{MHz})$, we obtained good results. From the measurement shown at $52 \mathrm{MHz}$ in Fig. 32, we can see that we get purely differential signals. The signals have approximately the same magnitude and are $180^{\circ}$ phase inverted. This means that the chip does work at very high frequencies.

We note in Fig. 32 that the output waveform had distortion at the peaks of the waveforms. For high-frequency AC signals, if the impedance at the output node is $50 \Omega$ and the capacitance is about $10 p F$, the large load will pull a very large current, the output buffer in the chip still has difficulty in driving the load (especially when $f>30 M \mathrm{~Hz}$ ). This is the reason why we see the distortion at the peak of the waveform.

We used a HP 4195A spectrum analyzer to verify the bandwidth of the circuit. Before we began the formal measurement, we did careful calibration for the $4195 \mathrm{~A}$ and kept the noise (from cable, test board, outside, etc.) very low (usually $<60 \mathrm{~dB}$ ) until very high frequencies (usually $>200 \mathrm{MHz}$ ). This is a very difficult task. One must make sure to have a very clean test board and very good calibration, even the length of test cable has a significant effect on the noise contribution. Fig. 33 shows the frequency response of the chip (on second test board). The output load is $50 \Omega$. The measurement probe adds about $10 p F$ load capacitance at the measured node. We can see that the bandwidth of the chip is above $130 \mathrm{MHz}$. After $130 \mathrm{MHz}$ the curve is changed by other parasitic capacitance. Fig. 34 shows the DC transfer curve of the converter. The output buffers of the chip have an offset, so the curve is not symmetric. We will analyze the offset in the next chapter. 


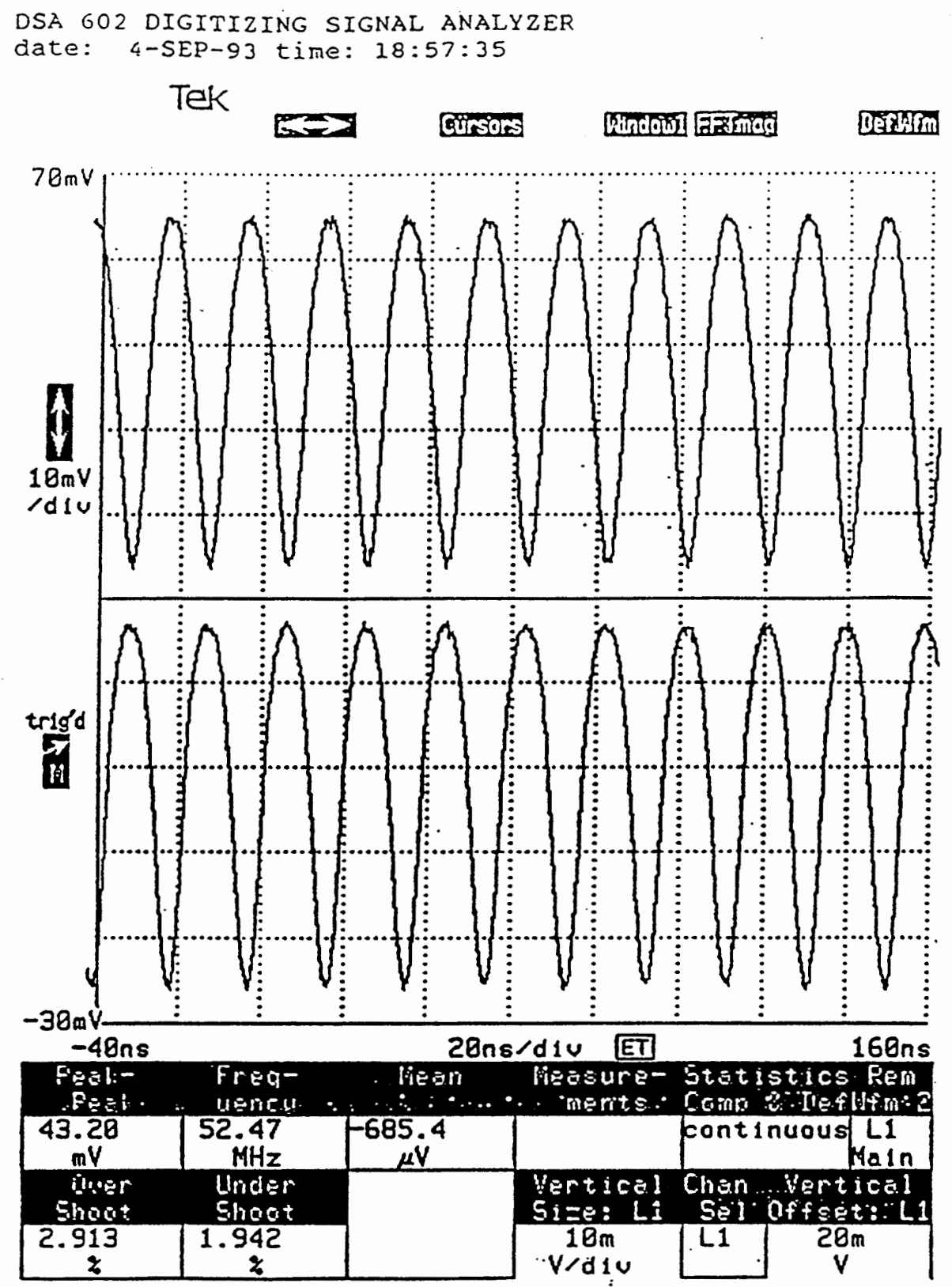

Figure. 32. The AC measurement on the $2 \mathrm{nd}$ test board at $f=52 \mathrm{MHz}$. 


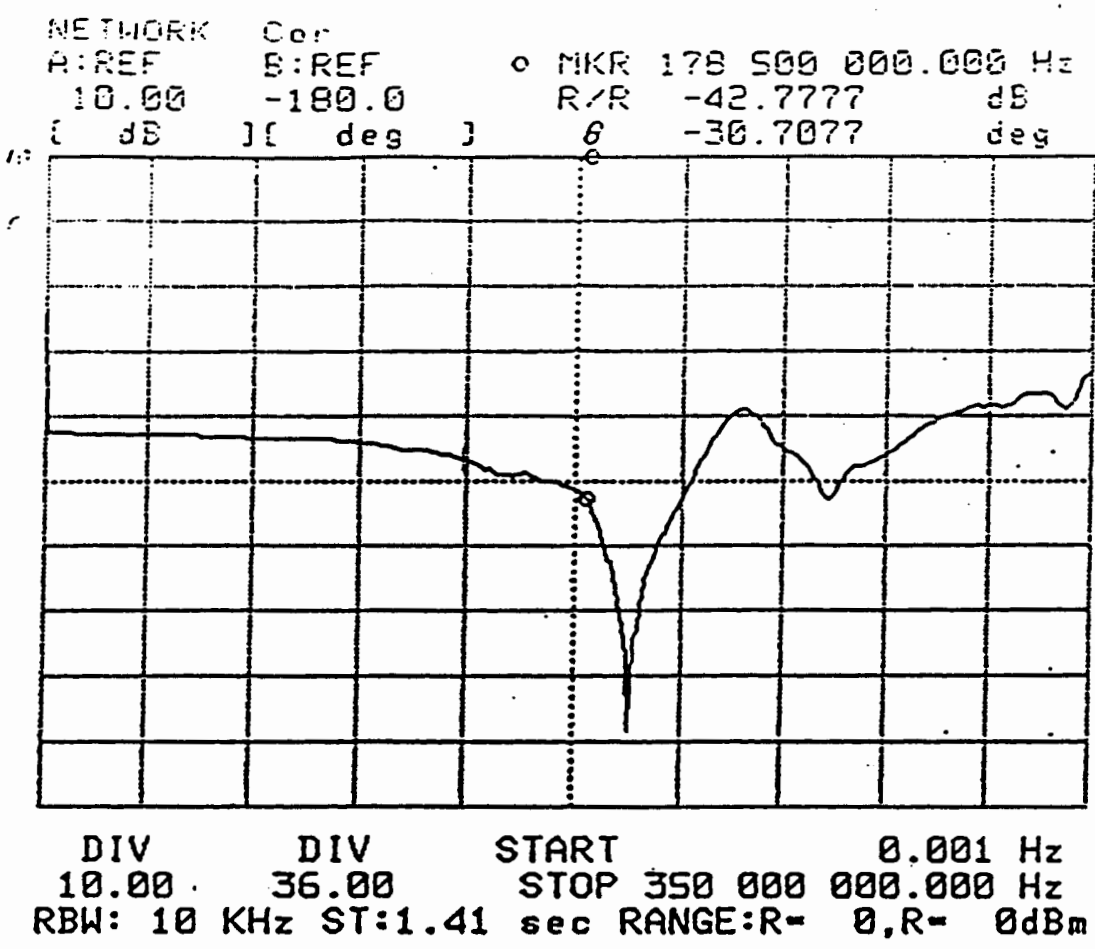

$$
\text { - }
$$

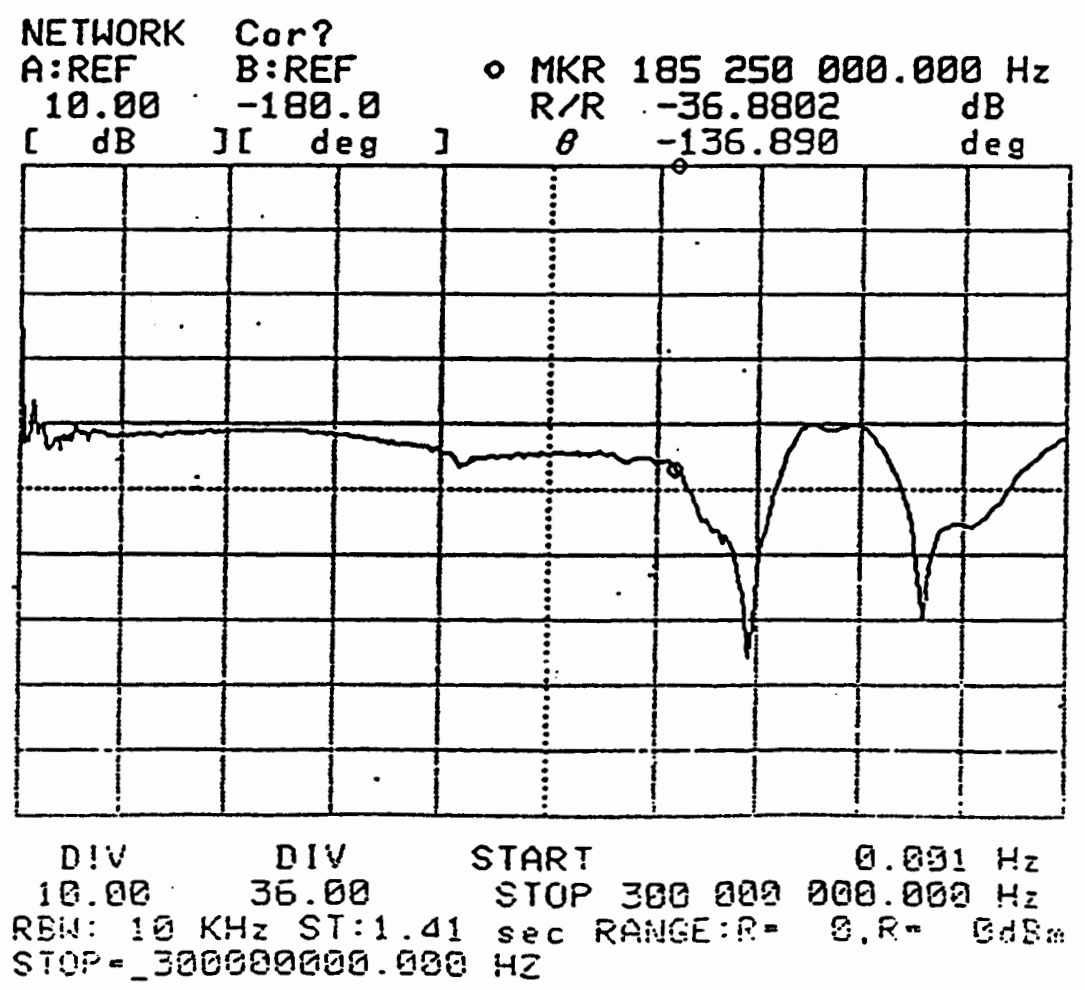

Figure. 33. The bandwidth measurement results of the chip. 


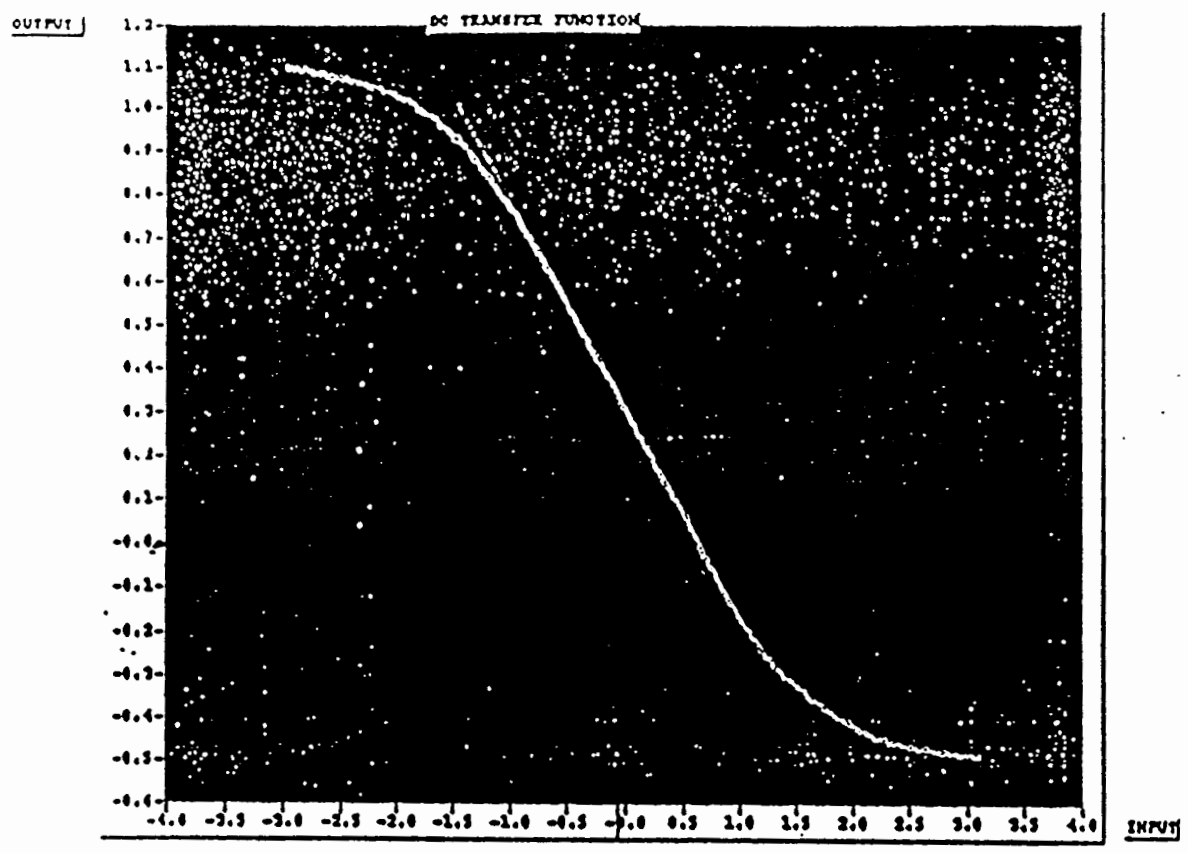

Figure. 34. DC transfer function measurement result of the chip.

By now we finished DC, AC and bandwidth testing for this chip. We can see that the measurements agree with the simulation results. As a new way to design integrated "voltage transformer", the chip design is successful.

The single-ended to differential voltage converter circuit and a differential to single-ended converter circuit (based on circuit in Fig. 13) were used in another filter chip (see Fig. 35). The frequency responses of the filter with external transformers (see measurement circuit in Fig. 1) and with the integrated converters are shown in Fig. 36 and 37 . We can see that because the external transformers can only work in a range (in our case is between $200 \mathrm{kHz}$ to $100 \mathrm{MHz}$ ), so at low-frequency range the circuit does not work. The intergrated converter can work in a large range (from DC to $100 \mathrm{MHz}$ ), but the signal loss is large. This is because our design problem (the gain is too small) and we can fix it easily. We will talk about the problem in the next chapter. 


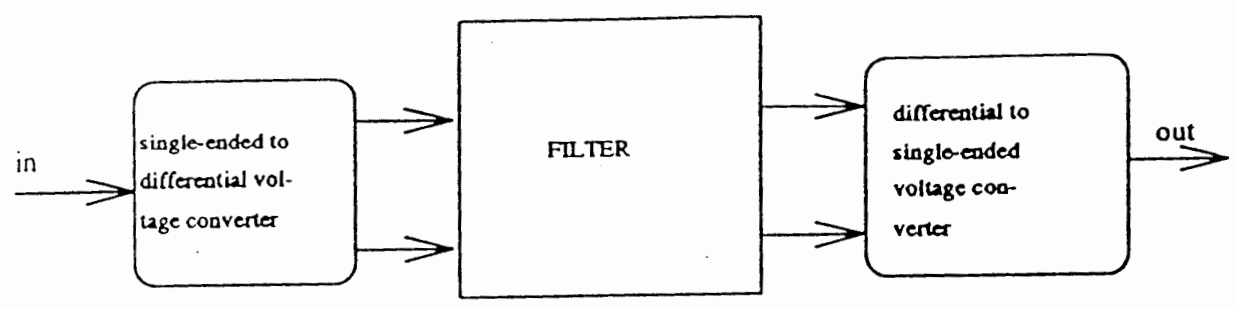

Figure. 35. The circuitry of filter with internal voltage converters.

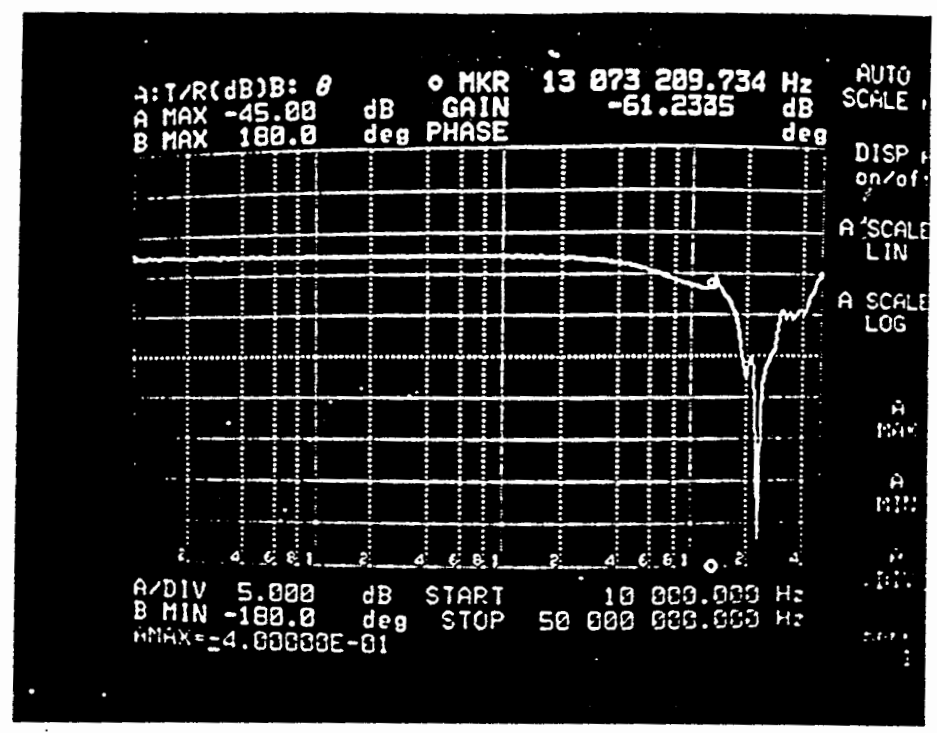

Figure. 37. The filter measurement $(\mathrm{AC})$ with internal voltage converters.

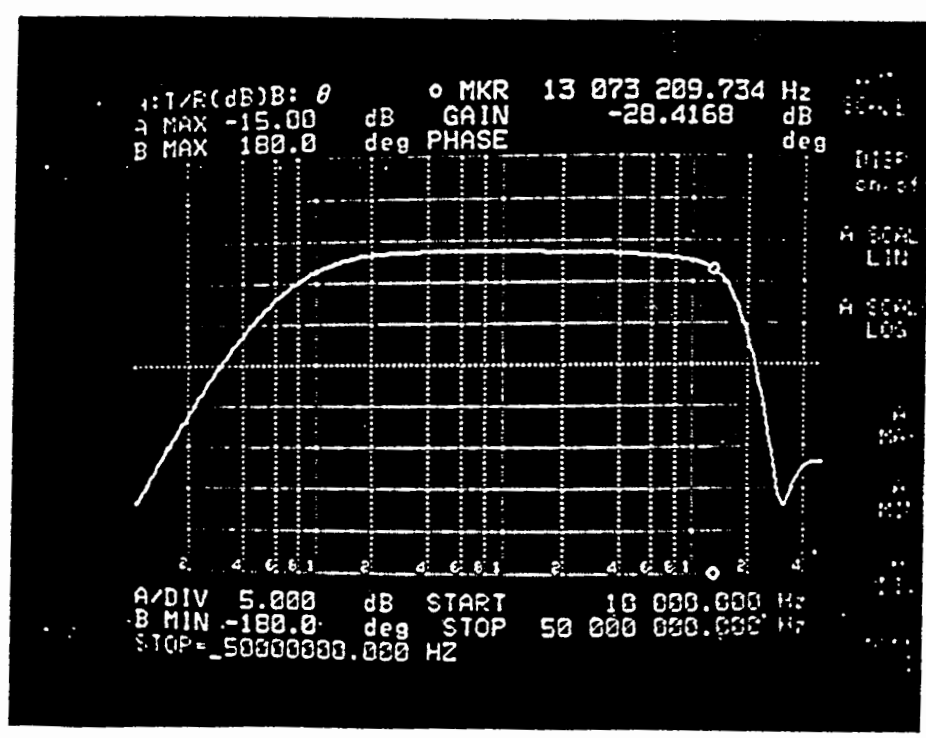

Figure. 36. The filter measurement $(\mathrm{AC})$ with extemal voltage transformers. 


\section{CHAPTER VII}

\section{CONCLUSIONS AND FUTURE WORK}

\section{Conclusion}

In this thesis, the design strategies for high-frequency OTA-based analog signal processing (ASP) systems have been presented. New techniques for the design of the basic building block, the operational transconductance amplifier (OTA), have been proposed with emphasis on linear input range and frequency response, with special attention paid to considerations for the high-frequency effects of parasitics. The circuits have been fabricated and evaluated experimentally.

First, based on very simple transconductance cells, cascade current mirrors were used to realize current copying and subtraction. A differential in/out OTA was built with large input range, good linearity and a high cut-off frequency $(>300 \mathrm{MHz})$. A single in/out transconductance cell with offset-reduced circuitry was designed, which can be used as a fast voltage inverter or a grounded-resistor.

High-frequency analog circuit measurement is extremely difficult compared with that of digital circuits. A high-frequency $\left(f_{-3 d B}>260 \mathrm{MHz}\right)$, good linearity CMOS buffer was designed and was used in the chip measurements.

Based on two initial designs of voltage converters, a cascade OTA voltage converter was created. The converter can provide pure differential voltage and can work in a range from $\mathrm{DC}$ to $100 \mathrm{MHz}$.

Carefully attention was paid in layout design of the chip, as well as the measurement set-up. The measurement results agree with the design simulations. The design is 
successful. The voltage converter circuit can be used widely in integrated circuits to replace discrete voltage transformers.

\section{Future Work}

The design of the converter and the buffer basically meet the requirements, but from the results of simulation and measurement, we still can find places for improvement. Here I discuss some problems found and what improvements can be made.

\section{The Output Buffer:}

As we discussed before, the buffer has an important role in all measurements. Because it is built in CMOS and the output transistor sizes are small, the output impedance is about $600 \Omega$. This is too large for many applications because in many cases the load is smaller than $100 \Omega$. In the experiments, we already saw the effect on AC and bandwidth measurements (the magnitude loss and the distortion). Also because in the chip we used a single-ended buffer instead of a differential buffer, the performance was worse (see Fig. 38 , there is a large offset). The drawback can be improved by enlarging the size of output stage transistors to enhance the driving ability. By this way we can get a $150 \Omega$ output impedance buffer without too much difficulty. The signal loss of the buffer will be improved from $-22 \mathrm{~dB}$ to about $-12 \mathrm{~dB}$. Smaller distortion also can be achieved. If we would build npn transistors in the chip, we can reduce the output impedance of the buffer dramatically, but that is a more complicated process (BiCMOS). Also the buffer had an offset because of body effect, we need a redesign to delete this offset.

A better way to overcome these shortcomings is to introduce feedback into the buffer circuit. Adding a feedback stage (see Fig. 39) improves the performance greatly over that of original design. The working principle is that if the output changes linearly following the input, the circuit works in exactly the same way as circuit in Fig. 17. But if the output does not change linearly, the feedback will force v(1a) to change, then the 


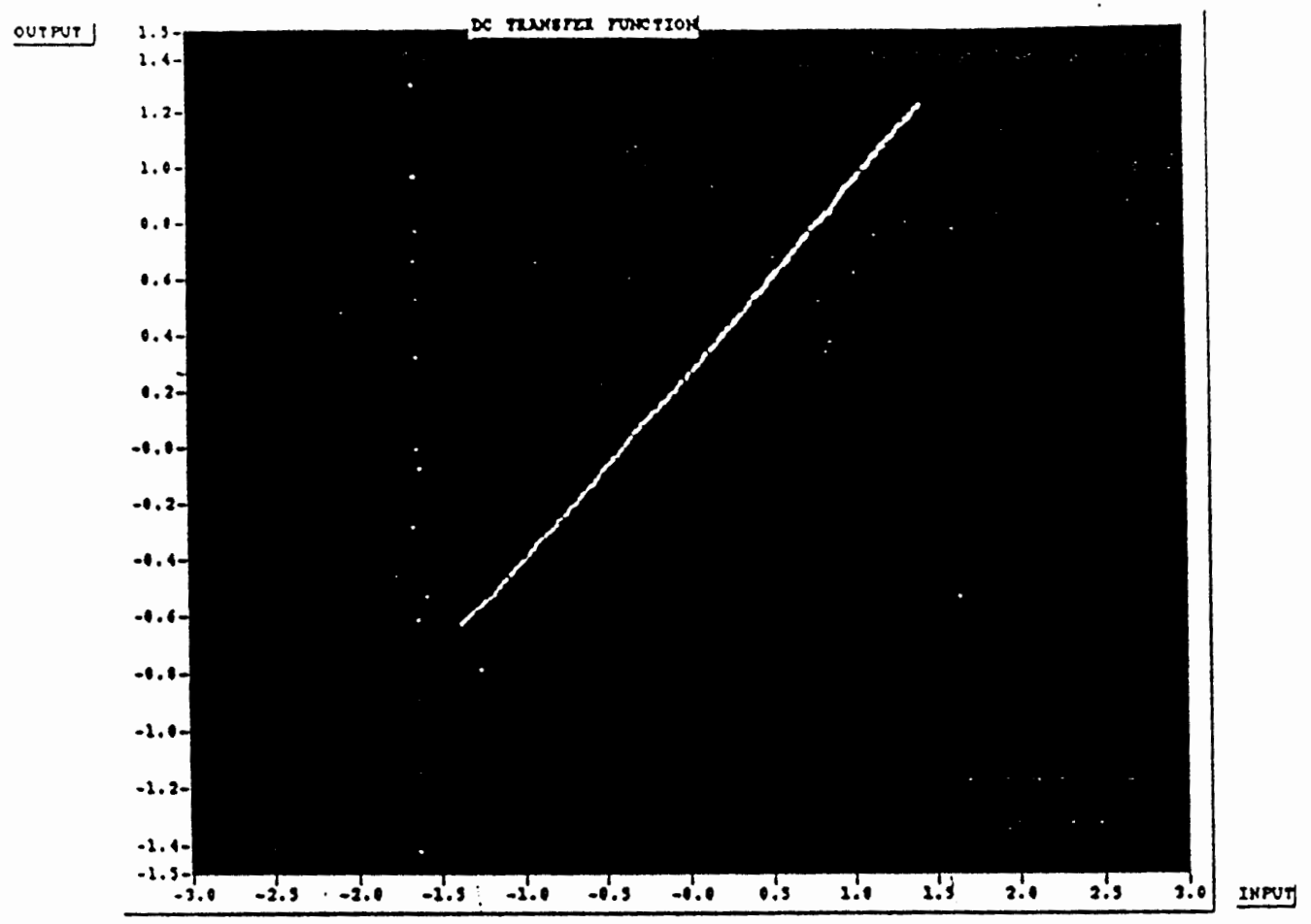

Figure. 38. The measurement (DC) of a single-ended buffer.

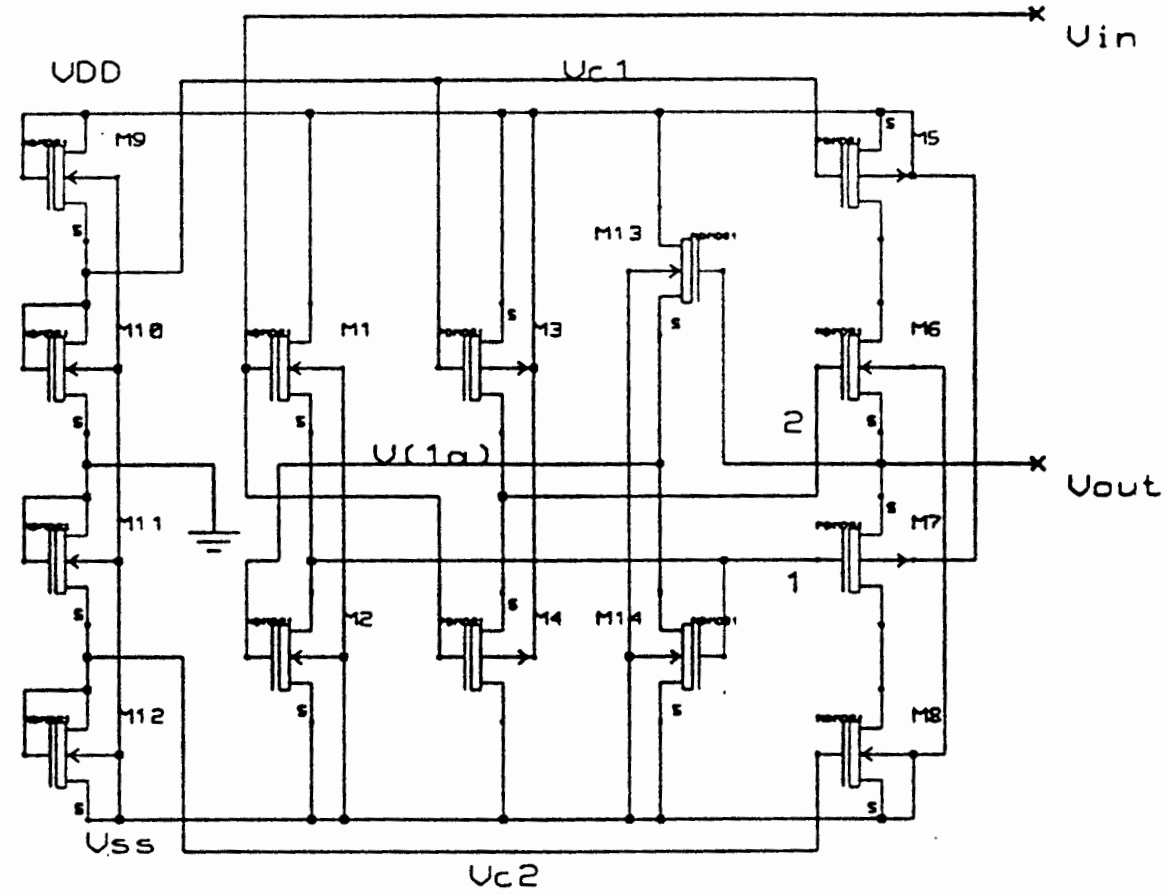

Figure. 39. A improved single-ended buffer. 
input stage bias voltage is changed to correct the non-linearity. The voltage gain is also increased because assuming the feedback stage gain is A, from eq. (3.7),

$$
A_{v}=\frac{g_{m 4}}{\frac{2 g_{d s} 3}{A}+g_{m 4}} \frac{R_{L}}{R_{L}+\frac{1}{A} \frac{1}{\left(g_{m 6}+g_{m 7}\right)}}
$$

For same size devices, the output impedance decreases from $600 \Omega$ to $100 \Omega$. The offset changes from $60 \mathrm{mV}$ to $10 \mathrm{mV}$. The gain rises from 0.82 to 0.93 (see Fig. 40 ). The frequency (with load $100 \mathrm{k} \Omega, 1 \mathrm{pF}$ ) increases from $260 \mathrm{MHz}$ to $360 \mathrm{MHz}$. The input range is a little smaller than before. So for the next chip design, we can use better output buffers.

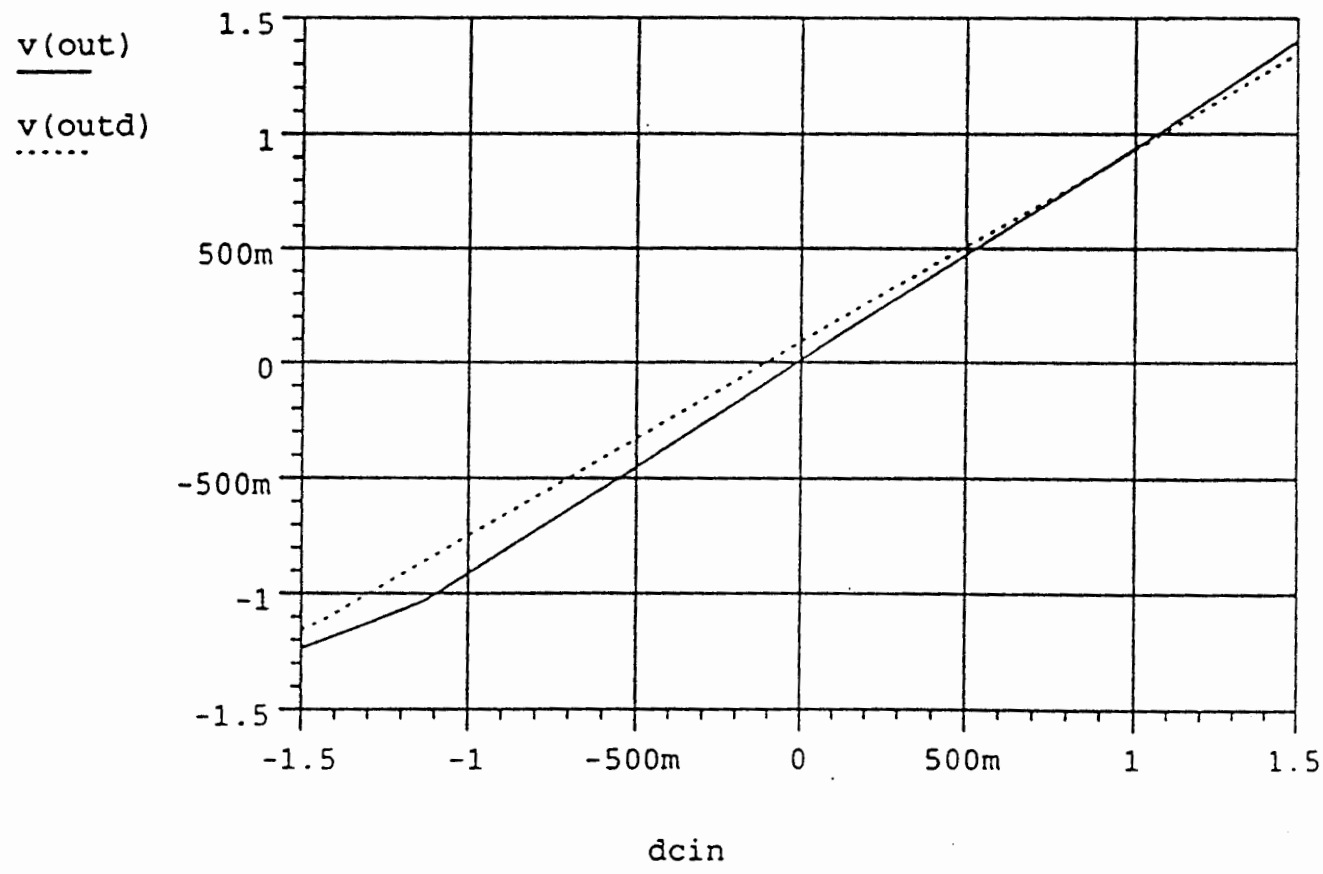

Figure. 40. The DC transfer functions of the original single-ended buffer and the improved buffer. $v($ outd) is the output of original buffer and $v(o u t)$ is output of the improved buffer. 


\section{OTA-resistor}

The second problem of the voltage converter chip arises because the resistor we designed is too small, the dc gain of the voltage converter is smaller than 0.5 . We need to change transistors' sizes for this OTA-resistor so we can get a dc gain around unity. The resistor also has offset problem. How to reduce the offset and, at the same time, keep the resistor's simple circuitry (small parasitic capacitance) and good linearity requires more work.

\section{Others:}

Because we did not know the MOSIS process well, some mistakes were made when we designed the layout of the chip. E.g., in this process, Pin1 is connected to the substrate of the chip $\left(V_{S S}\right)$. Having ignored this in our design, Pin 1 was one output of part of the circuits. This mistake caused the failure of a part of the circuit, and resulted in damage to the first two chips during the measurement.

For this very-high-frequency circuit design, the current and voltage gain are low, the whole circuit is very simple, and there is no feedback, so this design also depends on circuit fabrication process. For next design we can think about introducing feedback for the whole circuit to reduce the process effect. 


\section{REFERENCES}

[1] R. Schaumann, "Design of Continuous-time Fully Integrated Filters: a Review," IEE Proceedings, Vol. 136, Pt. G, No. 4, August 1989, pp. 184-190.

[2] J. S. Martinez, M. S. J. Steyaert, " A 10.7-MHz 68-dB SNR CMOS ContinuousTime Filter with On-Chip Automatic Tuning", IEEE J. Solid-State Circuits, Vol. 27, No. 12, DECEMBER 1992, pp. 1843-1853.

[3] B. Stefanelli and A. Kaiser, "A 2- $\mu \mathrm{m}$ CMOS Fifth-Order Low-Pass ContinuousTime Filter for Video-Frequency Applications", IEEE J. Solid-State Circuits, Vol. 28, No. 7, July 1993, pp. 713-718.

[4] W. M. Snelgrove, and A. Shoval, "A Balanced 0.9- $\mu$ CMOS Transconductance-C Filter Tunable Over the VHF Range," IEEE J. Solid-State Circuits, Vol. 27, No. 3, March, 1992, pp. 314-323.

[5] Bram Nauta, "A CMOS Transconductance-C Filter Technique for Very High Frequencies," IEEE J. Solid-State Circuits, Vol. 27, No. 2, February 1992, pp. 142 153.

[6] S. C. Huang and M. Ismail, "Linear Tunable Comfet Transconductor," Electronics Letters, Vol. 29, No. 5, 4th March 1993, pp. 459-461.

[7] A. Guzinski and T. Kulej, "New Fully-Balanced OTA structure," Electronics Letters, Vol. 28, No. 5, February 1992, pp. 498-499.

C. S. Park and R. Schaumann, "A High-Frequency CMOS Linear Transconduc- 
tance Element," IEEE Transactions on Circuits and System, Vol. CAS-33, No. 11, November 1986, pp. 1132-1138.

[9] M. F. Li, X. Chen and Y. C. Lim, "Linearity Improvement of CMOS Transconductors for Low Supply Applications," Electronics Letters, Vol. 29, No. 12, 10th June 1993, pp. 1106-1107.

[10] G. Wilson and P. K. Chan, "Floating CMOS Resistor," Electronics Letters, Vol. 29, No. 3, 4th February 1993, pp. 306-307.

[11] K. Nay and A. Budak, "A Voltage Controled Resistance with Wide Dynamic Range and Low Distortion," IEEE Trans. Circuits and Systems, Vol. CAS-30, No.10, Oct. 1983, pp. $770-772$.

[12] Nosratinia, A., Ahmadi, M., Jullien, G. A., and Shridhar, M.: "High-Drive CMOS Buffer For Large Capacitive Loads", Electron. Lett., 1991, 27, pp. 1044-1046

[13] Fisher J. A., and Koch, R.: "A High Linear CMOS Buffer Amplifiers", IEEE J. Solid-State Circuits, 1987 , SC-22, pp. 330-334

[14] Nagaraj, K.: "Large-swing CMOS Buffer Amplifier", IEEE J. Solid-State Circuits. 1989, SC-24, pp. 181-183

[15] P. E. Allen and D. R. Holberg, "CMOS Analog Circuit Design," New York, Holt, Rinehart and Winston, 1987.

[16] Y. Tsividis, "Operation and Modeling of the MOS Transistors," New York, McGraw-Hill, 1986.

[17] N. Weste and K. Eshraghian, "Principles of CMOS VLSI Design," AddisonWesley, 1985. 
[18] R. L. Geiger, P. E. Allen and N. R. Strader, "VLSI Design Techniques for Analog and Digital Circuits," McGraw-Hill, 1990.

[19] L. A. Glasser and D. W. Dobberpuhl, "The Design and Analysis of VLSI Circuits," Addison-Wesley, 1985.

[20] P. Larsson and C. Svensson, "Measuring high-bandwidth signals in CMOS circuits," Electronics Letters, Vol. 29, No. 20, 30th September 1993, pp. 1761-1762.

[21] P. Wu, "The Design of High-Frequency Continuous-Time Intrgrated Analog Signal Processing Circuits," Ph.D thesis, Portland State University, Portland, Oregon, Dec., 1993.

[22] A. Rothermel and F. Dell'ova, "Analog Phase Measuring Circuit for Digital CMOS IC's," IEEE J. Solid-State Circuits, Vol. 28, No. 7, July 1993, pp. 853-856. 


\section{APPENDIX I}

POST PROCESS MOSIS N14Q SPICE MODEL

N14Q SPICE LEVEL 2 PARAMETERS

.MODEL CMOSN NMOS LEVEL $=2$ LD $=0.250000 U$ TOX $=418.000008 \mathrm{E}-10$

$+\mathrm{NSUB}=9.2303604 \mathrm{E}+14 \mathrm{VTO}=0.85514 \mathrm{KP}=5.0471000 \mathrm{E}-05 \mathrm{GAMMA}=0.1954$

$+\mathrm{PHI}=0.6 \mathrm{UO}=596.714 \mathrm{UEXP}=0.075874 \mathrm{UCRIT}=10266.7$

+ DELTA $=2.733586$ VMAX $=65701.8 \mathrm{XJ}=0.250000 \mathrm{U}$ LAMBDA $=1.8428428 \mathrm{E}-02$

$+\mathrm{NFS}=1.085836 \mathrm{E}+12 \mathrm{NEFF}=1 \mathrm{NSS}=1.000000 \mathrm{E}+10 \mathrm{TPG}=1.000000$

$+\mathrm{RSH}=28.800000 \mathrm{CGDO}=3.099249 \mathrm{E}-10 \mathrm{CGSO}=3.099249 \mathrm{E}-10 \mathrm{CGBO}=3.830243 \mathrm{E}-10$

$+\mathrm{CJ}=8.997 \mathrm{E}-04 \mathrm{MJ}=0.557810 \mathrm{CJSW}=5.527200 \mathrm{E}-10 \mathrm{MJSW}=0.285976 \mathrm{~PB}=0.8000$

00

*Weff $=$ Wdrawn - Delta_W

* The suggested Delta_W is -0.24 um

.MODEL CMOSP PMOS LEVEL $=2 \mathrm{LD}=0.250000 \mathrm{U}$ TOX $=418.000008 \mathrm{E}-10$

$+\mathrm{NSUB}=9.30800 \mathrm{E}+15 \mathrm{VTO}=-0.889827 \mathrm{KP}=1.9068000 \mathrm{E}-05 \mathrm{GAMMA}=0.6249$

$+\mathrm{PHI}=0.6 \mathrm{UO}=216.495 \mathrm{UEXP}=0.218488 \mathrm{UCRIT}=62664$

+ DELTA $=0.169261$ VMAX $=100000.1 \mathrm{XJ}=0.250000 \mathrm{U} \mathrm{LAMBDA}=5.019540 \mathrm{E}-02$

$+\mathrm{NFS}=9.265210 \mathrm{E}+11 \mathrm{NEFF}=1.001 \mathrm{NSS}=1.000000 \mathrm{E}+10 \mathrm{TPG}=-1.000000$

$+\mathrm{RSH}=66.820000 \mathrm{CGDO}=3.098661 \mathrm{E}-10 \mathrm{CGSO}=3.098661 \mathrm{E}-10 \mathrm{CGBO}=3.726660 \mathrm{E}-10$

$+\mathrm{CJ}=2.980500 \mathrm{E}-04 \mathrm{MJ}=0.555355 \mathrm{CJSW}=3.0047600 \mathrm{E}-10 \mathrm{MJSW}=0.287927 \mathrm{~PB}=0.8000$

00

* Weff $=$ Wdrawn - Delta_W

* The suggested Delta_W is -0.17 um 


\begin{tabular}{|c|c|c|}
\hline \multicolumn{2}{|c|}{ Model Parameters } & Units \\
\hline LD & lateral diffusion (length) & meter \\
\hline TOX & oxide thickness & meter \\
\hline NSUB & substrate doping density & $1 / \mathrm{cm}^{3}$ \\
\hline VTO & zero-bias threshold voltage & volt \\
\hline $\mathrm{KP}$ & transconductance & amp/volt ${ }^{2}$ \\
\hline GAMMA & bulk threshold parameter & volt 0.5 \\
\hline $\mathrm{PHI}$ & surface potential & volt \\
\hline UO & surface mobility & $\mathrm{cm}^{2} /$ volt $\mathrm{sec}$ \\
\hline UEXP & mobility degradation exponent & \\
\hline UCRIT & mobility degradation critical field & $\mathrm{volt} / \mathrm{cm}$ \\
\hline DELTA & width effect on threshold & \\
\hline VMAX & maximum drift velocity & meter/sec \\
\hline $\mathrm{XJ}$ & metallurgical junction depth & meter \\
\hline LAMBDA & channel-length modulation & volt ${ }^{-1}$ \\
\hline NFS & fast surface state density & $1 / \mathrm{cm}^{2}$ \\
\hline NEFF & channel charge coefficient & \\
\hline NSS & surface state density & $1 / \mathrm{cm}^{2}$ \\
\hline TPG & $\begin{array}{l}\text { gate material type: } \\
+1=\text { opposite of substrate } \\
-1=\text { same as substrate } \\
0=\text { aluminum }\end{array}$ & \\
\hline RSH & drain, source diffusion sheet resistance & $\mathrm{ohm} / \mathrm{square}$ \\
\hline CGDO & gate-drain overlap capacitance/channel with & farad/meter \\
\hline CGSO & gate-source overlap capacitance/channel with & farad/meter \\
\hline CGBO & gate-bulk overlap capacitance/channel with & farad/meter \\
\hline CJ & bulk p-n zero-bias bottom capacitance/area & faradmeter ${ }^{2}$ \\
\hline MJ & bulk p-n bottom grading coefficient & \\
\hline CJSW & bulk p-n zero-bias perimeter capacitance/length & farad/meter \\
\hline MJSW & bulk p-n sidewall grading coefficient & \\
\hline PB & bulk ohmic resistance & $\mathrm{ohm}$ \\
\hline
\end{tabular}




\section{APPENDIX II}

\section{NETLISTS OF SOME CIRCUITS}

\section{A. The Netlist of Differential Input/Out OTA in Fig. 9}

m1 vdd $6 \mathrm{M}$ vss $\mathrm{cmosn}: \mathrm{w}=4 \mathrm{u} \mathrm{l}=2 \mathrm{u}$

$\mathrm{m} 2$ vdd $10 \mathrm{~N}$ vss cmosn: $\mathrm{w}=4 \mathrm{u} \mathrm{l}=2 \mathrm{u}$

m3 16 vss vss cmosn : $w=4 u l=2 u$

m4 $1 \mathrm{~N}$ vss vss cmosn: $\mathrm{w}=4 \mathrm{u} \mathrm{l}=2 \mathrm{u}$

m5 $5 \mathrm{M}$ vss vss $\mathrm{cmosn}: \mathrm{w}=4 \mathrm{u} \mathrm{l}=2 \mathrm{u}$

m6 510 vss vss $\mathrm{cmosn}: \mathrm{w}=4 \mathrm{u} \mathrm{l}=2 \mathrm{u}$

$\mathrm{m} 7 \mathrm{M}$ vc vss vss $\mathrm{cmosn}: \mathrm{w}=8 \mathrm{u} l=2 \mathrm{u}$

$\mathrm{m} 8 \mathrm{~N}$ vc vss vss $\mathrm{cmosn}: \mathrm{w}=8 \mathrm{u} \mathrm{l}=2 \mathrm{u}$

m5a 117 vdd cmosp : w=48u l=2u

m6a 2118 vdd cmosp: $w=12 \mathrm{u} \quad \mathrm{l}=2 \mathrm{u}$

m7a 2518 a vdd cmosp: $w=12 \mathrm{u} \quad \mathrm{l}=2 \mathrm{u}$

m8a 559 vdd cmosp: $w=48 \mathrm{u} \mathrm{l}=2 \mathrm{u}$

m9 $359 \mathrm{a}$ vdd cmosp : w=12u $\quad \mathrm{l}=2 \mathrm{u}$

m10 $1259 \mathrm{~b}$ vdd cmosp: $w=12 \mathrm{u} \quad l=2 \mathrm{u}$

m11 $211212 a$ vss cmosn: $w=16 u l=2 u$

m12 252515 vss cmosn: $w=16 u l=2 u$

m13 $32515 a$ vss cmosn : $w=16 u l=2 u$

m14 121213 vss cmosn: $w=16 \mathrm{u}$ l=2u

m15 77 vdd vdd cmosp: $w=48 u \quad l=2 u$

m1699 vdd vdd cmosp: $w=48 u \quad l=2 u$ 
m17 9a 9 vdd vdd cmosp $: w=12 u \quad l=2 u$

m18 9b 9 vdd vdd cmosp : $w=12 \mathrm{u} \quad \mathrm{l}=2 \mathrm{u}$

m19 87 vdd vdd cmosp:w $=12 u \quad l=2 u$

m20 8a 7 vdd vdd cmosp: $w=12 u \quad l=2 u$

m21 1313 vss vss cmosn:w=16u l=2u

$\mathrm{m} 2215 \mathrm{a} 15$ vss vss $\mathrm{cmosn}: \mathrm{w}=16 \mathrm{u} \mathrm{l}=2 \mathrm{u}$

m23 1515 vss vss cmosn:w=16u $l=2 u$

$\mathrm{m} 2412 \mathrm{a} 13$ vss vss $\mathrm{cmosn}: \mathrm{w}=16 \mathrm{u} \mathrm{l}=2 \mathrm{u}$

B. Netlist of Offset-Reduced $G_{m}$ Cell in Fig. 14

vin in $0 \mathrm{v}: \mathrm{dc}=\mathrm{dcin}$

vvdd vdd $0 \mathrm{v}: \mathrm{dc}=5$

vvss vss $0 \mathrm{v}: \mathrm{dc}=-5$

$\mathrm{sa}$ in out $\mathrm{s} 2: \mathrm{p} 1=30 \mathrm{u}$

sc 0 outa s 2 :p1 $=30 \mathrm{u}$

rout out $0 \mathrm{r}: \mathrm{r}=1 \mathrm{k}$

rout1 outoff $0 \mathrm{r}: r=1 \mathrm{k}$

cout out $0 \mathrm{c}: \mathrm{c}=30 \mathrm{f}$

coutoff outoff $0 \mathrm{c}: \mathrm{c}=30 \mathrm{f}$

$\mathrm{m} 1 \mathrm{vdd}$ vdd $1 \mathrm{a}$ vss $\mathrm{cmosn}: \mathrm{w}=30 \mathrm{u} \mathrm{l}=2 \mathrm{u}$

m2 $1 \mathrm{a}$ in outoff vdd cmosp:w $=30 \mathrm{ul}=2 \mathrm{u}$

$\mathrm{m} 3$ outoff in $2 \mathrm{a}$ vss $\mathrm{cmosn}: \mathrm{w}=30 \mathrm{u} \mathrm{l}=2 \mathrm{u}$

$\mathrm{m} 4$ vss vss $2 \mathrm{a}$ vdd cmosp:w $=30 \mathrm{u} \mathrm{l}=2 \mathrm{u}$

mla outa outa vdd vdd cmosp: $w=14 \mathrm{u} l=40 \mathrm{u}$

m2a out outa vdd vdd cmosp:w $=15 \mathrm{u} l=40 \mathrm{u}$ 
$\mathrm{m} 3 \mathrm{a}$ outa outa vss vss $\mathrm{cmosn}: \mathrm{w}=14 \mathrm{u} l=40 \mathrm{u}$

m4a out outa vss vss cmosn: $w=15 \mathrm{u} l=40 \mathrm{u}$

model $\mathrm{s} 2$ subckt:nodes=(in out) p1

vvdd vdd $0 \mathrm{v}: \mathrm{dc}=5$

vvss vss $0 \mathrm{v}: \mathrm{dc}=-5$

m1 vdd vdd 1 vss cmosn:w=p1 l=2u

$\mathrm{m} 21$ in out vdd cmosp:w=p1 $1=2 u$

$\mathrm{m} 3$ out in 2 vss cmosn:w=p1 $1=2 u$

$\mathrm{m} 4$ vss vss 2 vdd cmosp:w=p $1 \mathrm{l}=2 \mathrm{u}$

endm

C. Netlist of Differential Buffer

$\mathrm{m} 9$ vdd vdd 1 vss $\mathrm{cmosn}: \mathrm{w}=20 \mathrm{u} \mathrm{l}=2 \mathrm{u}$

m9a 112 vss cmosn:w=27u l=2u

m10 223 vss cmosn: $w=20 u l=2 u$

m10a 330 vss cmosn:w $=20 \mathrm{u} l=2 \mathrm{u}$

m11 004 vss cmosn:w=20u l=2u

m11a 445 vss cmosn:w=20u l=2u

m12 556 vss $\operatorname{cmosn}: w=12 u l=2 u$

m12a 66 vss vss $\mathrm{cmosn}: \mathrm{w}=20 \mathrm{u} l=2 \mathrm{u}$

$\mathrm{m} 1 \mathrm{vdd}$ in 1 vss cmosn:w=30u $\mathrm{l}=2 \mathrm{u}$

$\mathrm{m} 21 \mathrm{vc}$ vss vss cmosn:w $=30 \mathrm{u} l=2 \mathrm{u}$

$\mathrm{m} 3$ vss in 2 vdd cmosp:w $=10 \mathrm{ul} l=2 \mathrm{u}$

m4 2 vc1 vdd vdd cmosp: $w=10 \mathrm{u} \mathrm{l}=2 \mathrm{u}$

m5 3 vc1 vdd vdd cmosp: $w=60 u l=2 u$ 
m6 32 outa vss cmosn: $w=80 \mathrm{u} \mathrm{l}=2 \mathrm{u}$

m7 41 outa vdd cmosp:w=120u $l=2 u$

m8 4 vc vss vss $\quad$ cmosn:w $=80 \mathrm{u} l=2 u$

m1a vdd ina $1 \mathrm{a}$ vss $\mathrm{cmosn}: \mathrm{w}=30 \mathrm{u} l=2.0 \mathrm{u}$

m2a 1 a ve vss vss cmosn: $w=30 \mathrm{u} l=2.0 \mathrm{u}$

$\mathrm{m} 3 \mathrm{a}$ vss ina $2 \mathrm{a}$ vdd $\mathrm{cmosp}: \mathrm{w}=10 \mathrm{u} \mathrm{l}=2.0 \mathrm{u}$

m4a 2 a vc1 vdd vdd cmosp: $w=10 \mathrm{u} 1=2.0 \mathrm{u}$

m5a 3 a vc1 vdd vdd cmosp:w $=60 \mathrm{u} l=2.0 \mathrm{u}$

m6a 3 a 2 a out vss cmosn: $w=80 \mathrm{ul}=2.0 \mathrm{u}$

$\mathrm{m} 7 \mathrm{a} 4 \mathrm{a}$ 1a out vdd $\mathrm{cmosp}: \mathrm{w}=120 \mathrm{u} \mathrm{l}=2.0 \mathrm{u}$

m8a $4 \mathrm{a}$ vc vss vss $\quad \mathrm{cmosn}: \mathrm{w}=80 \mathrm{u} \mathrm{l}=2.0 \mathrm{u}$

D. Netlist of Single-Ended Improved Buffer

$\mathrm{m} 9$ vdd vdd 1 vss $\mathrm{cmosn}: \mathrm{w}=20 \mathrm{u} l=2 \mathrm{u}$

m9a 112 vss cmosn:w=27u l=2u

m10 223 vss cmosn: $w=20 u l=2 u$

m10a 330 vss cmosn:w $=20 \mathrm{u} \mathrm{l}=2 \mathrm{u}$

m11 004 vss $\mathrm{cmosn}: \mathrm{w}=20 \mathrm{ul}=2 \mathrm{u}$

m11a 445 vss cmosn:w $=20 \mathrm{u} l=2 \mathrm{u}$

m12 556 vss cmosn:w $=12 \mathrm{ul}=2 \mathrm{u}$

m12a 66 vss vss cmosn:w=20u $1=2 u$

$\mathrm{m} 1$ vdd in 1 vss $\mathrm{cmosn}: \mathrm{w}=40 \mathrm{ul}=2 \mathrm{u}$

m2 1 1a vss vss cmosn:w $=40 \mathrm{u} l=2 \mathrm{u}$

M13 vdd out 1 a vss cmosn: $w=40 u l=2 u$

m14 1a 1 vss vss cmosn: $w=40 u l=2 u$ 
$\mathrm{m} 3$ vss in 2 vdd cmosp: $\mathrm{w}=10 \mathrm{u} \mathrm{l}=2 \mathrm{u}$

m4 2 vc1 vdd vdd cmosp: $w=10 \mathrm{u} 1=3 \mathrm{u}$

;m3a 2a 2 vdd vdd cmosp: $w=10 \mathrm{u} l=3 \mathrm{u}$

;m4a vss out $2 \mathrm{a}$ vdd $\mathrm{cmosp}: \mathrm{w}=10 \mathrm{u} \mathrm{l}=2 \mathrm{u}$

m5 3 vc1 vdd vdd cmosp: $w=30 u l=2 u$

m6 32 out vss cmosn: $w=40 \mathrm{u} l=2 \mathrm{u}$

m7 41 out vdd cmosp:w=60u l=2u

m8 4 vc vss vss cmosn: $w=40 u l=2 u$

;m5a 3 vcl vdd vdd cmosp:w=100u l=2u

;m6a 32 out vss cmosn:w=200u l=2u

;m7a 41 out vdd cmosp:w=300u l=2u

;m8a 4 vc vss vss cmosn: $w=200 u l=2 u$ 